SPECTRAL ANALYSIS OF BRAGG AND NON-BRAGG ORDERS IN DYNAMIC HOLOGRAPHY USING PHOTOREFRACTIVE MATERIALS

\author{
Thesis \\ Submitted to \\ The School of Engineering of the \\ UNIVERSITY OF DAYTON \\ In Partial Fulfillment of the Requirements for \\ The Degree of \\ Master of Science in Electrical Engineering \\ By \\ Akash Kota \\ UNIVERSITY OF DAYTON \\ Dayton, Ohio \\ August, 2016
}


SPECTRAL ANALYSIS OF BRAGG AND NON-BRAGG ORDERS IN DYNAMIC HOLOGRAPHY USING PHOTOREFRACTIVE MATERIALS

Name: Kota, Akash

APPROVED BY:

Partha P. Banerjee, Ph.D.

Advisory Committee Chairman

Professor

Department of Electrical and

Computer Engineering

Department Chair

Department of Electro-Optics

and Photonics
Monish R. Chatterjee, Ph.D.

Committee Member

Professor

Department of Electrical and

Computer Engineering

Joseph W. Haus, Ph.D.

Committee Member

Professor

Department of Electro-Optics

and Photonics

Robert J. Wilkens, Ph.D., P.E.

Associate Dean for Research and Innovation

Professor

School of Engineering
Eddy M. Rojas, Ph.D., M.A., P.E.

Dean

School of Engineering 
CCopyright by

Akash Kota

All rights reserved

2016 


\section{ABSTRACT \\ SPECTRAL ANALYSIS OF BRAGG AND NON-BRAGG ORDERS IN DYNAMIC HOLOGRAPHY USING PHOTOREFRACTIVE MATERIALS}

Name: Kota, Akash

University of Dayton

Advisor: Partha P. Banerjee

The photorefractive effect is a nonlinear optical effect that refers to change in refractive index of a material when it is illuminated by light. When illuminated by an interference pattern of coherent light source, this PR effect is responsible for two-beam coupling in PR materials, sometimes leading to energy exchange between the beams. PR materials can also be used as holographic storage media. In fact, dynamic real-time holographic interferometry can be implemented using photorefractive materials. To achieve this, two beams, one called the pump and one called the object beam, are introduced onto a photorefractive material to write the hologram of the object. During the hologram writing process, these beams can couple in intensity and/or phase which thereafter are responsible for self-diffraction of these beams, and can also give rise to Bragg and non-Bragg orders. The information from the Bragg and non-Bragg orders plays an important role in determining the $3 \mathrm{D}$ information of the object. In this thesis, an exact study is performed to examine the spatial evolution of Bragg and non-Bragg orders in photorefractive iron doped lithium niobate for different types of beam profiles such as Gaussian and flattops using an angular plane wave spectral decomposition technique. For Gaussian beam incidence, it has been found that higher or non-Bragg 
orders shows evidence of mode conversion of incident beam profiles. The numerical technique developed in this work should be useful in determining the phases of the Bragg and non-Bragg orders which have applications in dynamic phase-shifting digital holography and holographic interferometry. 


\section{ACKNOWLEDGMENTS}

I would like to specially thank my advisor Dr. Partha P. Banerjee, for directing this thesis and for all his help, motivation, time, patience, encouragement, careful attention, support and advice in my studies. I would also like to thank my committee members Dr. Monish Chatterjee for his encouragement and support, and Dr. Joseph Haus for his support and helpful comments.

Additionally, I also would like to specially thank Dr. Guru Subramanyam for their encouragement, Dr. Bradley Duncan for his support, and Dr. Tarek Taha, Dr. Eric Balster, and Dr. Elena Guliants for their inspirational classes. I would also like to thank my research group members Dr. Ujitha Abeywickrema, Dr. Han Li, Rudra Gnawali, Mahmudunnabi Basunia, Diane Beamer, and Arjun Krishnappa for their valuable time and support.

Also I would like to specially thank my friends Suman Chaparala, Kiran Kadiyala and Anup Josyula for their encouragement and support. Finally I would like to thank my parents, cousins, brothers and sisters for being my strength and standing beside me all the time. 


\section{TABLE OF CONTENTS}

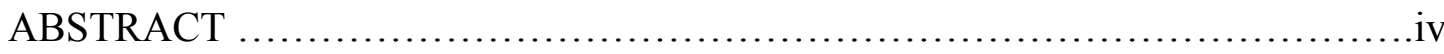

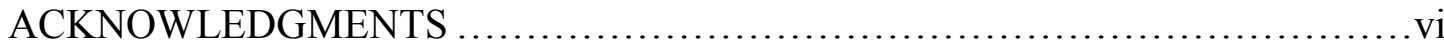

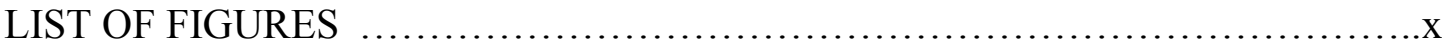

LIST OF ABBREVIATIONS ..............................................

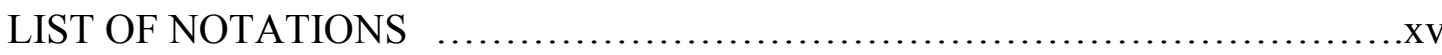

CHAPTER I INTRODUCTION AND OBJECTIVES ...........................

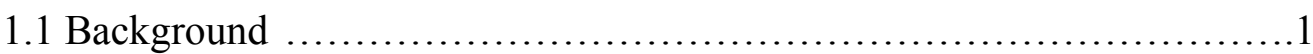

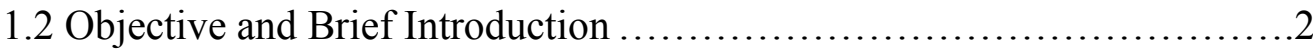

1.3 Thesis Outline .........................................................

CHAPTER II INTERACTION OF ANGULAR PLANE WAVE SPECTRA OF BRAGG AND NON-BRAGG ORDERS IN PHOTOREFRACTIVE LITHIUM NIOBATE ..........................................5

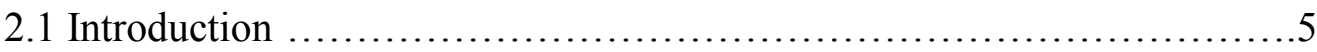

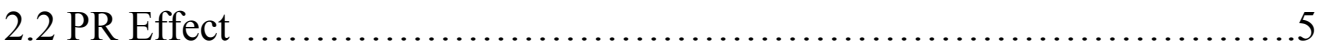

2.3 Experimental Demonstration of Bragg and Non-Bragg Orders in PR LN .7

2.4 Derivation of Spatial Evolution of Bragg and Non-Bragg Orders for Interacting Plane Waves ..........................................

2.5 Theoretical Model for Interacting Angular Spectra ....................11

2.6 Derivation of Photovoltaic Coefficient $\left(c_{1}\right)$ Using Kukhtarev Equations

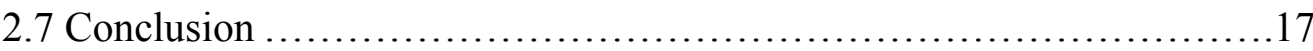

CHAPTER III SPECTRAL ANALYSIS OF BRAGG AND NON-BRAGG ORDERS USING GAUSSIAN BEAM PROFILES ...............18

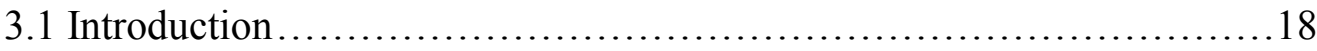

3.2 Bragg and Non-Bragg Orders for Identical Incident Gaussian Beam

Profiles .18

3.2.1 Numerical results for $P_{1}=P_{-1}=20 \mathrm{~mW} ; W_{1}=W_{-1}=$ $0.4 \mathrm{~mm} ; \theta=0.5^{\circ}$ .20

3.3 Bragg and Non-Bragg Orders for Incident Gaussian Beam Profiles with

Different Powers and Identical Waists .26 
3.3.1 Numerical results for $P_{1}=20 \mathrm{~mW}, P_{-1}=10 \mathrm{~mW} ; W_{1}=$ $W_{-1}=0.4 \mathrm{~mm} ; \theta=0.5^{\circ}$.

3.3.2 Numerical results for $P_{1}=20 \mathrm{~mW}, P_{-1}=2 \mathrm{~mW}$;

$$
W_{1}=W_{-1}=0.4 \mathrm{~mm} ; \theta=0.5^{\circ} \text {. }
$$

3.4 Bragg and Non-Bragg Orders for Incident Gaussian Beam Profiles

with Different Waists

3.4.1 Numerical results for $W_{1}=1.6 \mathrm{~mm}, W_{-1}=0.4 \mathrm{~mm} ; P_{1}=$ $P_{-1}=20 \mathrm{~mW} ; \theta=0.5^{\circ}$

3.4.2 Numerical results for $W_{1}=2.4 \mathrm{~mm}, W_{-1}=0.3 \mathrm{~mm} ; P_{1}=$ $P_{-1}=20 \mathrm{~mW} ; \theta=0.5^{\circ}$ .34

3.5 Bragg and Non-Bragg Orders for Incident Gaussian Beam Profiles with Different Interaction Angles: Intensity and Phase

3.5.1 Numerical results for $P_{1}=P_{-1}=20 \mathrm{~mW} ; W_{1}=W_{-1}=$ $2.4 \mathrm{~mm} ; \theta=0.5^{\circ}$

3.5.2 Numerical results for $P_{1}=P_{-1}=20 \mathrm{~mW} ; W_{1}=W_{-1}=$ $2.4 \mathrm{~mm} ; \theta=1^{\circ}$

3.5.3 Numerical results for $P_{1}=P_{-1}=20 \mathrm{~mW} ; W_{1}=W_{-1}=$ $2.4 \mathrm{~mm} ; \theta=2^{\circ}$

3.5.4 Numerical results for $P_{1}=P_{-1}=20 \mathrm{~mW} ; W_{1}=W_{-1}=$ $0.6 \mathrm{~mm} ; \theta=2^{\circ}$

3.6 Conclusion

\section{CHAPTER IV SPECTRAL ANALYSIS OF BRAGG AND NON-BRAGG}

ORDERS USING ARBITRARY BEAM PROFILES

4.1 Introduction

4.2 Bragg and Non-Bragg Orders for Different Incident Flattop

Beam Profiles

4.2.1 Numerical results for $P_{1}=P_{-1}=20 \mathrm{~mW} ; W_{1}=3.6 \mathrm{~mm}$, $W_{-1}=0.6 \mathrm{~mm} ; \theta=0.5^{\circ}$

4.2.2 Numerical results for $P_{1}=P_{-1}=20 \mathrm{~mW} ; W_{1}=W_{-1}=$ $2.4 \mathrm{~mm} ; \theta=2^{\circ}$

4.3 Numerical Analysis by Varying the Incident Beam Profiles .55

4.3.1 Numerical results for $P_{1}=P_{-1}=20 \mathrm{~mW} ; W_{1}=3.6 \mathrm{~mm}$, $W_{-1}=0.6 \mathrm{~mm} ; \theta=0.5^{\circ}$.

4.3.2 Numerical results for $P_{1}=P_{-1}=20 \mathrm{~mW} ; W_{1}=W_{-1}=$ $2.4 \mathrm{~mm} ; \theta=2^{\circ}$

4.4 Conclusion

5.1 Conclusion

5.2 Future Work

BIBLIOGRAPHY

APPENDIX .66 
Selected MATLAB ${ }^{\odot}$ Codes Used in This Work............................66 


\section{LIST OF FIGURES}

Figure 2.1: Illustration of PR effect. (a) PR material illuminated with an interference pattern of coherent light source, (b) excitation of photo generated electrons into conduction band, (c) generation of charge carriers in a PR material, (d) Distribution of charge carriers in a PR material, (e) creation of space charge field inside a PR material, (f) modulation of refractive index due to EO effect in a PR material

Figure 2.2: Experimental setup for recording the hologram in LN crystal. The $\operatorname{Bragg}\left(E_{1}, E_{-1}\right)$ and first two non-Bragg $\left(E_{3}, E_{-3}\right)$ orders are shown to the left of the figure [14]

Figure 2.3: Nine non-Bragg orders seen during self-diffraction in PR LN [14].

Figure 3.1: Geometrical representation of Bragg and non-Bragg orders propagating in PR LN.

Figure 3.2: Gaussian beam profiles of incident (a) pump (reference) beam and (b) probe (object) beams. $P_{1}=20 \mathrm{~mW}, W_{1}=0.4 \mathrm{~mm} ; P_{-1}=20 \mathrm{~mW}, W_{-1}=$ $0.4 \mathrm{~mm}$. The spatial shifts of $1 \mu \mathrm{m}$ is not discernible at this scale.

Figure 3.3: Spatial evolution of (a) first Bragg order $\left(E_{1}\right)$, (b) second Bragg order $\left(E_{-1}\right)$, (c) first non-Bragg order $\left(E_{3}\right)$, and (d) second non-Bragg order $\left(E_{-3}\right)$ in PR LN. $P_{1}=20 \mathrm{~mW}, W_{1}=0.4 \mathrm{~mm} ; P_{-1}=20 \mathrm{~mW}, W_{-1}=0.4 \mathrm{~mm} \ldots . .22$

Figure 3.4: Variation of on-axis $(x=0)$ intensity of (a) first Bragg order $\left(E_{1}\right)$, (b) second Bragg order $\left(E_{-1}\right)$, (c) first non-Bragg order $\left(E_{3}\right)$, and (d) second nonBragg order $\left(E_{-3}\right)$ during propagation. $\quad P_{1}=20 \mathrm{~mW}, W_{1}=0.4 \mathrm{~mm} ; P_{-1}=$ $20 \mathrm{~mW}, W_{-1}=0.4 \mathrm{~mm}$

Figure 3.5: (a) First Bragg order $E_{1}$ at the entrance plane (red) and exit plane (black) of PR LN; (b) second non-Bragg order $\left(E_{-3}\right)$ at exit plane. 
Figure 3.6: Far field pattern of the (a) first Bragg order $\left(E_{1}\right)$, (b) second Bragg order $\left(E_{-1}\right)$, (c) first non-Bragg order $\left(E_{3}\right)$, and (d) second non-Bragg order $\left(E_{-3}\right)$ after propagating a distance of $50 \mathrm{~cm}$ from exit plane of PR LN crystal.

Figure 3.7: Gaussian beam profiles of incident (a) pump (reference) and (b) probe (object) beams. $P_{1}=20 \mathrm{~mW}, W_{1}=0.4 \mathrm{~mm} ; P_{-1}=10 \mathrm{~mW}, W_{-1}=0.4 \mathrm{~mm}$. The spatial shifts of $1 \mu \mathrm{m}$ is not discernible at this scale.

Figure 3.8: Spatial evolution of (a) first Bragg order $\left(E_{1}\right)$, (b) second Bragg order $\left(E_{-1}\right)$, (c) first non-Bragg order $\left(E_{3}\right)$, and (d) second non-Bragg order $\left(E_{-3}\right)$ in PR LN. $P_{1}=20 \mathrm{~mW}, W_{1}=0.4 \mathrm{~mm} ; P_{-1}=10 \mathrm{~mW}, W_{-1}=0.4 \mathrm{~mm}$

Figure 3.9: Gaussian beam profiles of incident (a) pump (reference) and (b) probe (object) beams. $\quad P_{1}=20 \mathrm{~mW}, W_{1}=0.4 \mathrm{~mm} ; P_{-1}=2 \mathrm{~mW}, W_{-1}=$ $0.4 \mathrm{~mm}$. The spatial shifts of $1 \mu \mathrm{m}$ is not discernible at this scale. 30

Figure 3.10: Spatial evolution of (a) first Bragg order $\left(E_{1}\right)$, (b) second Bragg order $\left(E_{-1}\right)$, (c) first non-Bragg order $\left(E_{3}\right)$, and (d) second non-Bragg order $\left(E_{-3}\right)$ in PR LN. $P_{1}=20 \mathrm{~mW}, W_{1}=0.4 \mathrm{~mm} ; P_{-1}=2 \mathrm{~mW}, W_{-1}=0.4 \mathrm{~mm}$

Figure 3.11: Gaussian beam profiles of incident (a) pump (reference) and (b) probe (object) beams. $P_{1}=20 \mathrm{~mW}, W_{1}=1.6 \mathrm{~mm} ; P_{-1}=20 \mathrm{~mW}, W_{-1}=$ $0.4 \mathrm{~mm}$. The spatial shifts of $1 \mu \mathrm{m}$ is not discernible at this scale.

Figure 3.12: Spatial evolution of (a) first Bragg order $\left(E_{1}\right)$, (b) second Bragg order $\left(E_{-1}\right)$, (c) first non-Bragg order $\left(E_{3}\right)$, and (d) second non-Bragg order $\left(E_{-3}\right)$ in PR LN. $P_{1}=20 \mathrm{~mW}, W_{1}=1.6 \mathrm{~mm} ; P_{-1}=20 \mathrm{~mW}, W_{-1}=0.4 \mathrm{~mm}$

Figure 3.13: Gaussian beam profiles of incident (a) pump (reference) and (b) probe (object) beams. $P_{1}=20 \mathrm{~mW}, W_{1}=2.4 \mathrm{~mm} ; P_{-1}=20 \mathrm{~mW}, W_{-1}=$ $0.3 \mathrm{~mm}$. The spatial shifts of $1 \mu \mathrm{m}$ is not discernible at this scale.

Figure 3.14: Spatial evolution of (a) first Bragg order $\left(E_{1}\right)$, (b) second Bragg $\operatorname{order}\left(E_{-1}\right)$, (c) first non-Bragg order $\left(E_{3}\right)$, and (d) second non-Bragg order $\left(E_{-3}\right)$ in PR LN. $P_{1}=20 \mathrm{~mW}, W_{1}=2.4 \mathrm{~mm} ; P_{-1}=20 \mathrm{~mW}, W_{-1}=0.3 \mathrm{~mm}$

Figure 3.15: Spatial evolution of (a) first Bragg order $\left(E_{1}\right)$, (b) second Bragg $\operatorname{order}\left(E_{-1}\right)$, (c) first non-Bragg order $\left(E_{3}\right)$, and (d) second non-Bragg order $\left(E_{-3}\right)$ in PR LN. $P_{1}=20 \mathrm{~mW}, W_{1}=2.4 \mathrm{~mm} ; P_{-1}=20 \mathrm{~mW}, W_{-1}=$ $2.4 \mathrm{~mm} ; \theta=0.5^{\circ}$ 
Figure 3.16: Unwrapped on-axis $(x=0)$ phase profile of (a) first Bragg order $\left(E_{1}\right)$, (b) second Bragg order $\left(E_{-1}\right)$, (c) first non-Bragg order $\left(E_{3}\right)$, and (d) second non-Bragg order $\left(E_{-3}\right)$ during propagation

Figure 3.17: Spatial evolution of (a) first Bragg order $\left(E_{1}\right)$, (b) second Bragg order $\left(E_{-1}\right)$, (c) first non-Bragg order $\left(E_{3}\right)$, and (d) second non-Bragg order $\left(E_{-3}\right)$ in PR LN. $P_{1}=20 \mathrm{~mW}, W_{1}=2.4 \mathrm{~mm} ; P_{-1}=20 \mathrm{~mW}, W_{-1}=$ $2.4 \mathrm{~mm} ; \theta=1^{\circ}$.

Figure 3.18: Variation of on-axis $(x=0)$ intensity of (a) first non-Bragg order $\left(E_{3}\right)$, and (b) second non-Bragg order $\left(E_{-3}\right)$ during propagation.

Figure 3.19: Unwrapped on-axis $(x=0)$ phase profile of (a) first Bragg order $\left(E_{1}\right)$, (b) second Bragg order $\left(E_{-1}\right)$, (c) first non-Bragg order $\left(E_{3}\right)$, and (d) second non-Bragg order $\left(E_{-3}\right)$ during propagation.

Figure 3.20: Spatial evolution of (a) first Bragg order $\left(E_{1}\right)$, (b) second Bragg $\operatorname{order}\left(E_{-1}\right)$, (c) first non-Bragg order $\left(E_{3}\right)$, and (d) second non-Bragg order $\left(E_{-3}\right)$ in $\quad$ PR LN. $\quad P_{1}=20 \mathrm{~mW}, W_{1}=2.4 \mathrm{~mm} ; P_{-1}=20 \mathrm{~mW}, W_{-1}=$ $2.4 \mathrm{~mm} ; \theta=2^{\circ}$

Figure 3.21: Variation of on-axis $(x=0)$ intensity of (a) first non-Bragg order $\left(E_{3}\right)$, and (b) second non-Bragg order $\left(E_{-3}\right)$ during propagation. .43

Figure 3.22: Unwrapped on-axis $(x=0)$ phase profile of (a) first Bragg order $\left(E_{1}\right)$, (b) second Bragg order $\left(E_{-1}\right)$, (c) first non-Bragg order $\left(E_{3}\right)$, and (d) second non-Bragg order $\left(E_{-3}\right)$ during propagation.

Figure 3.23: Spatial evolution of (a) first Bragg order $\left(E_{1}\right)$, (b) second Bragg order $\left(E_{-1}\right)$ (c) first non-Bragg order $\left(E_{3}\right)$, and (d) second non-Bragg $\operatorname{order}\left(E_{-3}\right)$ in PR LN. $P_{1}=20 \mathrm{~mW}, W_{1}=0.6 \mathrm{~mm} ; P_{-1}=20 \mathrm{~mW}, W_{-1}=$ $0.6 \mathrm{~mm} ; \theta=2^{\circ}$

Figure 3.24: Variation of on-axis $(x=0)$ intensity of (a) first non-Bragg order $\left(E_{3}\right)$, and (b) second non-Bragg order $\left(E_{-3}\right)$ during propagation. .46

Figure 3.25: Unwrapped on-axis $(x=0)$ phase profile of (a) first Bragg order $\left(E_{1}\right)$, (b) second Bragg order $\left(E_{-1}\right)$ (c) first non-Bragg order $\left(E_{3}\right)$, and (d) second non-Bragg order $\left(E_{-3}\right)$ during propagation. 
Figure 4.1: Flattop beam profiles of incident (a) pump (reference) and (b) probe (object) beams. $P_{1}=20 \mathrm{~mW}, W_{1}=3.6 \mathrm{~mm} ; P_{-1}=20 \mathrm{~mW}, W_{-1}=0.6 \mathrm{~mm}$.

Figure 4.2: Spatial evolution of (a) first Bragg order $\left(E_{1}\right)$, (b) second Bragg order $\left(E_{-1}\right)$ (c) first non-Bragg order $\left(E_{3}\right)$, and $(\mathrm{d})$ second non-Bragg order $\left(E_{-3}\right)$ in PR LN. $P_{1}=20 \mathrm{~mW}, W_{1}=3.6 \mathrm{~mm} ; P_{-1}=20 \mathrm{~mW}, W_{-1}=0.6 \mathrm{~mm} ; \theta=0.5^{\circ}$

Figure 4.3: Spatial evolution of (a) first Bragg order $\left(E_{1}\right)$, (b) second Bragg order $\left(E_{-1}\right)$ (c) first non-Bragg order $\left(E_{3}\right)$, and (d) second non-Bragg order $\left(E_{-3}\right)$ in PR LN. $P_{1}=20 \mathrm{~mW}, W_{1}=2.4 \mathrm{~mm} ; P_{-1}=20 \mathrm{~mW}, W_{-1}=2.4 \mathrm{~mm} ; \theta=2^{\circ}$

Figure 4.4: Variation of on-axis $(x=0)$ intensity of (a) first non-Bragg order $\left(E_{3}\right)$, and (b) second non-Bragg order $\left(E_{-3}\right)$ during propagation.

Figure 4.5: Unwrapped on-axis $(x=0)$ phase profile of (a) first Bragg order $\left(E_{1}\right)$, (b) second Bragg order $\left(E_{-1}\right)$ (c) first non-Bragg order $\left(E_{3}\right)$, and (d) second non-Bragg order $\left(E_{-3}\right)$ during propagation.

Figure 4.6: Gaussian and flattop beam profiles of incident (a) pump (reference) and (b) probe (object) beams. $P_{1}=20 \mathrm{~mW}, W_{1}=3.6 \mathrm{~mm} ; P_{-1}=$ $20 \mathrm{~mW}, W_{-1}=0.6 \mathrm{~mm}$

Figure 4.7: Spatial evolution of (a) first Bragg order $\left(E_{1}\right)$, (b) second Bragg order $\left(E_{-1}\right)$ (c) first non-Bragg order $\left(E_{3}\right)$, and $(\mathrm{d})$ second non-Bragg order $\left(E_{-3}\right)$ in PR LN. $P_{1}=20 \mathrm{~mW}, W_{1}=3.6 \mathrm{~mm} ; P_{-1}=20 \mathrm{~mW}, W_{-1}=0.6 \mathrm{~mm} ; \theta=0.5^{\circ} \ldots . .56$

Figure 4.8: Spatial evolution of (a) first Bragg order $\left(E_{1}\right)$, (b) second Bragg order $\left(E_{-1}\right)(\mathrm{c})$ first non-Bragg order $\left(E_{3}\right)$, and $(\mathrm{d})$ second non-Bragg order $\left(E_{-3}\right)$ in PR LN. $P_{1}=20 \mathrm{~mW}, W_{1}=2.4 \mathrm{~mm} ; P_{-1}=20 \mathrm{~mW}, W_{-1}=2.4 \mathrm{~mm} ; \theta=2^{\circ}$

Figure 4.9: Variation of on-axis $(x=0)$ intensity of (a) first non-Bragg order $\left(E_{3}\right)$, and (b) second non-Bragg order $\left(E_{-3}\right)$ during propagation.

Figure 4.10: Unwrapped on-axis $(x=0)$ phase profile of (a) first Bragg order $\left(E_{1}\right)$, (b) second Bragg order $\left(E_{-1}\right)$ (c) first non-Bragg order $\left(E_{3}\right)$, and (d) second non-Bragg order $\left(E_{-3}\right)$ during propagation 


\section{LIST OF ABBREVIATIONS}

CCD

$\mathrm{DH}$

DHI

EO

LN

PR

PSDH

PTP

RHI

RI
Charge coupled device

Digital holography

Dynamic holographic interferometry

Electro-optic

Lithium niobate

Photorefractive

Phase shifting digital holography

Photothermoplastics

Real-time holographic interferometry

Refractive index 


\section{LIST OF NOTATIONS}

\begin{tabular}{|c|c|}
\hline$E_{1}$ & Field, +1 Bragg order \\
\hline$E_{-1}$ & Field, -1 Bragg order \\
\hline$E_{3}$ & Field, +3 non-Bragg order \\
\hline$E_{-3}$ & Field, -3 non-Bragg order \\
\hline$E_{1 i}^{\prime}$ & $i^{\text {th }}$ component, +1 Bragg order /angular spectrum \\
\hline$E_{-1 i}^{\prime}$ & $i^{\text {th }}$ component, -1 Bragg order /angular spectrum \\
\hline$E_{3 i}^{\prime}$ & $i^{\text {th }}$ component, +3 non-Bragg order /angular spectrum \\
\hline$E_{-3 i}^{\prime}$ & $i^{\text {th }}$ component, -3 non-Bragg order /angular spectrum \\
\hline$E_{S C}$ & Electrostatic space-charge field in PR crystal \\
\hline$P_{1}$ & Power of pump beam \\
\hline$P_{-1}$ & Power of probe beam \\
\hline$W_{1}$ & Waist of pump beam \\
\hline$W_{-1}$ & Waist of probe beam \\
\hline$\theta$ & External angle between pump beam and probe beam \\
\hline$\theta^{\prime}$ & Internal angle between pump beam and probe beam \\
\hline$c_{1}$ & Photovoltaic coefficient \\
\hline
\end{tabular}




\begin{tabular}{|c|c|}
\hline$c_{2}$ & Diffusion coefficient \\
\hline$k_{0}$ & Free space wave vector \\
\hline$n_{0}$ & Linear part of refractive index \\
\hline$\varphi_{1}$ & Phase, +1 Bragg order \\
\hline$\varphi_{-1}$ & Phase, -1 Bragg order \\
\hline$\varphi_{3}$ & Phase, +3 non-Bragg order \\
\hline$\varphi_{-3}$ & Phase, -3 non-Bragg order \\
\hline$*$ & Complex conjugate \\
\hline$\Delta n$ & Change in refractive index \\
\hline$K$ & Magnitude of the grating vector \\
\hline$L$ & Thickness of the material (LN crystal) \\
\hline$\Delta K$ & Magnitude of the fundamental spatial frequency \\
\hline & component \\
\hline$\pi$ & The circular constant, approximately 3.14159 \\
\hline$\partial$ & Partial derivative \\
\hline$\lambda$ & Wavelength \\
\hline$z$ & $\mathrm{z}$ coordinate, nominal direction of propagation \\
\hline$x$ & $\mathrm{x}$ coordinate \\
\hline$n$ & Refractive index \\
\hline$j$ & Imaginary number, $\sqrt{-1}$ \\
\hline
\end{tabular}


$\alpha$

S

$n_{e}$

$N_{D}^{+}$

$J$

$k$

$N_{D}$

$N_{A}$

$\gamma_{R}$

$D_{s}$

$\varepsilon_{S}$

$\mu$

$\beta$

I

$e$

$\varphi$

$r_{33}$

$n_{o}$

$\nabla$
Absorption coefficient

Ionization cross-section

Free electron density

Ionized donor concentration

Current density

Photovoltaic constant

Donor concentration

Acceptor concentration

Recombination rate

Diffusion constant

Static dielectric constant

Carrier mobility

Thermal excitation rate

Intensity distribution of optical field

Charge of electron, $1.6023 \times 10^{-19} \mathrm{C}$

Phase

Electro-optic coefficient

Ordinary refractive index

Gradient 


\section{CHAPTER I}

\section{INTRODUCTION AND OBJECTIVES}

\subsection{Background}

Holography and digital holography (DH) have many applications in science and engineering, especially in the recreation and display of 3D images. Apart from applications in defense, they have numerous applications in industry, security, gaming, broadcast, medicine and forensics. For instance, recently a novel technique is being developed to identify the third level of details such as width and depth of pore on a forensically developed fingerprint sample. 3D imaging of such minor detail plays an important role in identifying criminals [1]. DH is able to reconstruct the threedimensional surface by processing holographic data recorded on a detector such as a charge coupled device (CCD). Holographic data can also be recorded in a photorefractive (PR) material. In fact, dynamic holographic interferometry (DHI) or real-time holographic interferometry (RHI) can be implemented by using PR materials $[2,3]$. DHI has applications in both engineering and medicine. In engineering applications, DHI is used to assess the 3D nature of cracks on airborne objects that have encountered rain, snow etc. during flight [4]. In medicine, DHI is used in the analysis of fractures in head, femur, and other bones $[5,6]$. To implement DHI or RHI using PR materials, two beams, one called a pump and one called the object beam are introduced onto a PR material to write the hologram of the object. During the hologram writing process, these beams can couple in intensity and/or phase which thereafter are responsible for self-diffraction of these incident beams, and can also give rise to Bragg 
and non-Bragg orders [13]. The information from the Bragg and non-Bragg orders plays an important role in determining the $3 \mathrm{D}$ information of the object through a technique referred to as phase shifting digital holography (PSDH). In PSDH, the holograms of the object are recorded successively with reference waves of different phase shifts. This enables one to obtain the 3D information or depth of the object upon reconstruction of the holograms. In this thesis, a thorough numerical analysis is performed to study the spatial evolution of Bragg and non-Bragg orders in PR materials specifically in iron doped lithium niobate (LN) for different varieties of incident beam profiles such as Gaussian and flattops.

\subsection{Objective and Brief Introduction}

The objective of this thesis is to study the evolution of the Bragg and nonBragg orders during two beam coupling in a PR material using the angular spectrum approach. PR materials can be used for holographic storage. In PR materials, the refractive index changes when it is illuminated by a coherent light source or optical interference pattern due to space charge field created inside the material [7]. The PR effect was discovered in 1966 and it is observed in crystals such as $\mathrm{LN}\left(\mathrm{LiNbO}_{3}\right)$, barium titanate $\left(\mathrm{BaTiO}_{3}\right)$, potassium niobate $\left(\mathrm{KNbO}_{3}\right)$, etc. [16]. The PR effect is responsible for two beam coupling in PR materials which can generate Bragg and nonBragg orders. The mechanism of the evolution of higher diffraction orders in a PR material has been extensively studied [8]. The generation of the space charge field and the induced refractive index profile in a PR material can be modeled using the Kukhtarev equations [9]. The interaction equations of Bragg and non-Bragg orders in a PR material have been numerically solved for the case of plane waves. It is also shown that dynamic holography using a PR material provides a convenient way of implementing PSDH [10]. 
In this thesis, the interaction equations of Bragg and non-Bragg orders in a PR material are formulated for the case of arbitrary profiles which can be represented by their respective angular plane wave spectra, for the first time to the best of our knowledge. Furthermore, the solutions to these equations are obtained numerically by solving them in MATLAB $^{\odot}$ for different types of beam profiles such as Gaussian and flattops. A thorough numerical analysis is performed to determine the shapes of Bragg and non-Bragg orders during their evolution in PR LN for different types of incident beam profiles.

\subsection{Thesis Outline}

Chapter II in this thesis develops the interaction equations of Bragg and nonBragg orders in a PR material for the case of interacting angular plane wave spectra. The spatial evolution equations of Bragg and non-Bragg orders are derived in terms of the interactions between their angular spectra. In this model, the interactions between two Bragg orders and two non-Bragg orders are considered.

In Chapter III the interaction equations of Bragg and non-Bragg orders are numerically solved for different types of Gaussian beam profiles. The spatial evolution of Bragg and non-Bragg orders in a PR material depends on different incident beam parameters such as beam power, beam waist and the angle between the two incident beams and material parameters such as value of the photovoltaic coefficient $\left(c_{1}\right)$ etc. In this Chapter the interaction equations for the angular plane wave spectra are solved simultaneously in MATLAB $^{\odot}$ for different Gaussian beam profiles and the numerical results obtained are analyzed.

In Chapter IV the interaction equations of Bragg and non-Bragg orders are numerically solved for different kinds of super-Gaussian beam profiles, which tend to 
a rectangular or flat-top beam profile in the limit. Also in this Chapter a combination of super Gaussian and Gaussian beam profiles is used as incident beams to solve the interaction equations simultaneously in MATLAB ${ }^{\odot}$. Thorough numerical simulations are performed by using arbitrary incident beam profiles and the numerical results obtained are analyzed.

Chapter V concludes the thesis and provides a summary of ongoing and future work. 


\section{CHAPTER II}

\section{INTERACTION OF ANGULAR PLANE WAVE SPECTRA OF BRAGG AND NON-BRAGG ORDERS IN PHOTOREFRACTIVE LITHIUM NIOBATE}

\subsection{Introduction}

Many materials in nature have nonlinear optical properties. This nonlinearity can arise due to various effects, such as nonlinear electronic response or polarizability, photorefractivity or thermal effects [15]. The refractive index (RI) in some crystals changes when it is illuminated by an interference pattern of coherent light source due to space charge field created inside the material. This effect is called PR effect and it is discussed below in some detail. PR materials and photothermoplastics (PTP) can be used as holographic storage media [12]. During two beam coupling in a PR material, the change in refractive index resulting from the interference of two incident beams can generate diffracted Bragg and non-Bragg orders. In this Chapter a detailed theory is developed to understand the spatial evolution of Bragg and non-Bragg orders in a PR material.

\subsection{PR Effect}

To understand the theory of Bragg and non-Bragg orders in PR LN, it is necessary to examine the PR effect in closer detail. As stated earlier, the PR effect is the induced change in the RI of a material when it is illuminated by an interference pattern. This is illustrated in Figure 2.1. It can be explained in four steps:

(a) Photoexcitation. Photoexcitation of mobile charge carriers (e.g., electrons) when a PR material is illuminated with an alternating (bright and dark) 
interference pattern as shown in Figure 2.1(a). It is assumed that a PR material has predominantly donor and some acceptor impurities and they have energy levels somewhere between valence and conduction bands. When light is incident on a material (bright regions), the energy of the light is sufficient to raise the electrons to the conduction band as shown in Figure 2.1(b). Thus we have photo-generated charge carriers as shown in Figure 2.1(c).

(b) Charge transport. In the regions where the light is less intense little or no charge carriers are generated, whereas the charge carriers in the conduction band generated by intense light are free to move about the material due to diffusion as shown in Figure 2.1(d).

(c) Trapping. A donor atom which donates an electron becomes an ionized donor. The diffused electrons may recombine with the ionized donor (shallow traps) or the acceptor (deep traps). The trapped charge may remain trapped even after light is removed. The ionized donors and acceptors remain immobile in a PR material.

(d) Induced change in refractive index. Light intensity differences in a PR material resulting from the incident interference pattern leads to separation of electrons and positive charges which give rise to space charge distribution and hence an induced electrostatic field or space charge field as shown in Figure 2.1(e). PR materials also exhibit electro-optic (EO) effect. Materials exhibiting EO effect alter their refractive index in response to an electric field, as shown in Figure 2.1(f). 


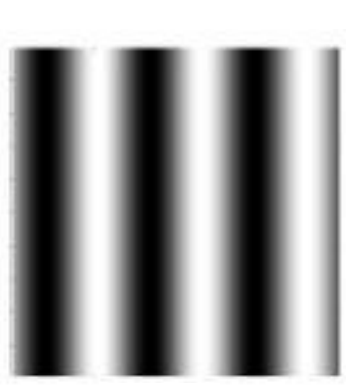

(a)

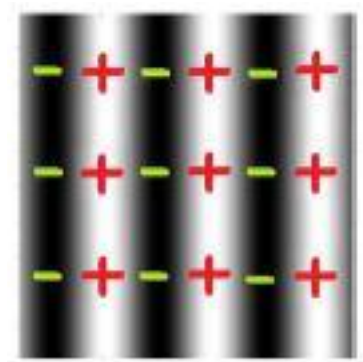

(d)

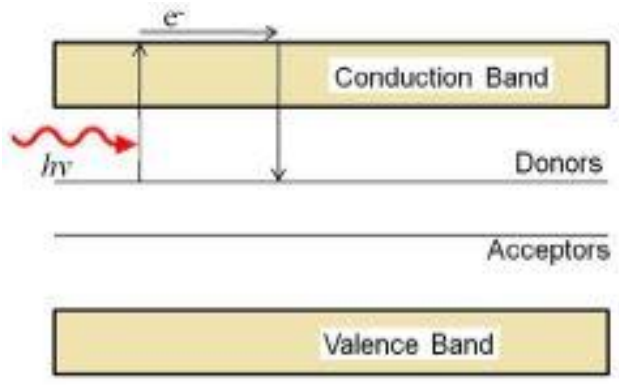

(b)

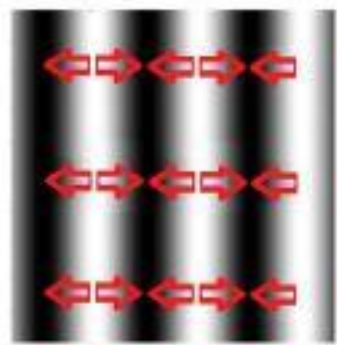

(e)

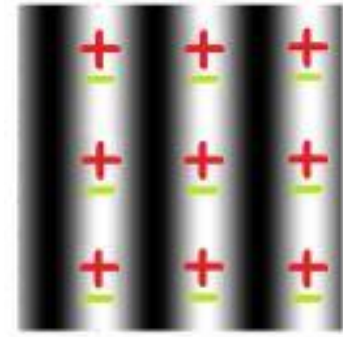

(c)

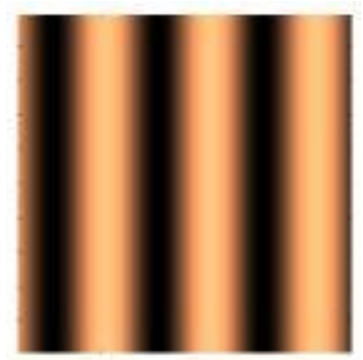

(f)

Figure 2.1: Illustration of PR effect. (a) PR material illuminated with an interference pattern of coherent light source, (b) excitation of photo generated electrons into conduction band, (c) generation of charge carriers in a PR material, (d) Distribution of charge carriers in a PR material, (e) creation of space charge field inside a PR material, (f) modulation of refractive index due to EO effect in a PR material.

The generation of the space charge field $E_{S C}$ and the induced refractive index $\Delta n$ profile in a PR material can be modeled using Kukhtarev equations [9]. The change in refractive index $\Delta n$ is expressed as

$$
\Delta n \propto E_{S C}=c_{1} I+c_{2} \frac{\partial I}{\partial x}
$$

where $I$ is the total intensity, $c_{1}$ and $c_{2}$ are the coefficients representing the photovoltaic and diffusive effects respectively in the PR medium [14]. Note that the second term on the RHS of eq.(2.1) represents a component of the more general intensity gradient.

\subsection{Experimental Demonstration of Bragg and Non-Bragg Orders in PR LN}

An experiment to demonstrate the generation of Bragg and non-Bragg orders in a PR material has been conducted in our lab. The experimental setup is shown in Figure 
2.2. Iron doped $\mathrm{LN}$ is used as the PR material. A $514.5 \mathrm{~nm}$ Ar-ion laser beam with power of approximately $40 \mathrm{~mW}$ is split into two beams. One of these beams is used to illuminate the object. The reflected or scattered beam from the object and the second beam (called the reference), is interfered in the crystal. A grating is then formed in the crystal by the coupling of two beams, thereafter which are responsible for selfdiffraction giving rise to generation of Bragg and non-Bragg orders [11]. A flat mirror has been first used as the "object" for simplicity. Also it is not always required, before starting the experiment the crystal is heated carefully to erase residual gratings. The external angle $\theta$ between the two beams is taken as $2^{\circ} . P_{1}, W_{1}$ represent the power and waist of the pump (reference) beam. Similarly $P_{-1}, W_{-1}$ represent the power and waist of the probe (object) beam. Multiple non-Bragg orders are observed as shown in Figure 2.3 .

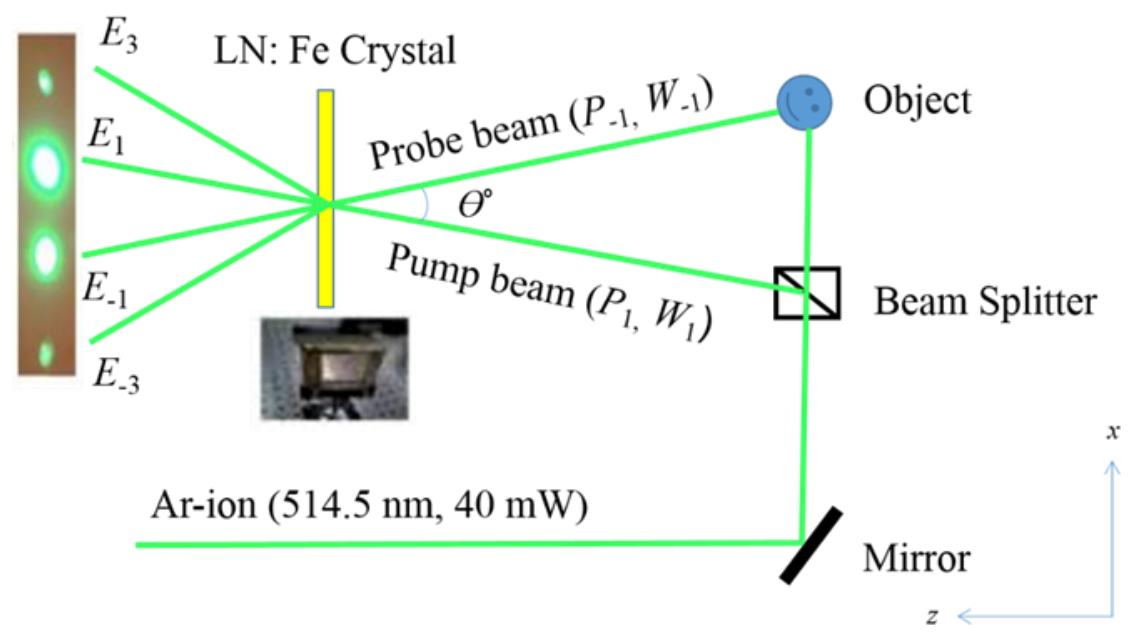

Figure 2.2: Experimental setup for recording the hologram in LN crystal. The Bragg $\left(E_{1}, E_{-1}\right)$ and first two non-Bragg $\left(E_{3}, E_{-3}\right)$ orders are shown to the left of the figure [14]. 


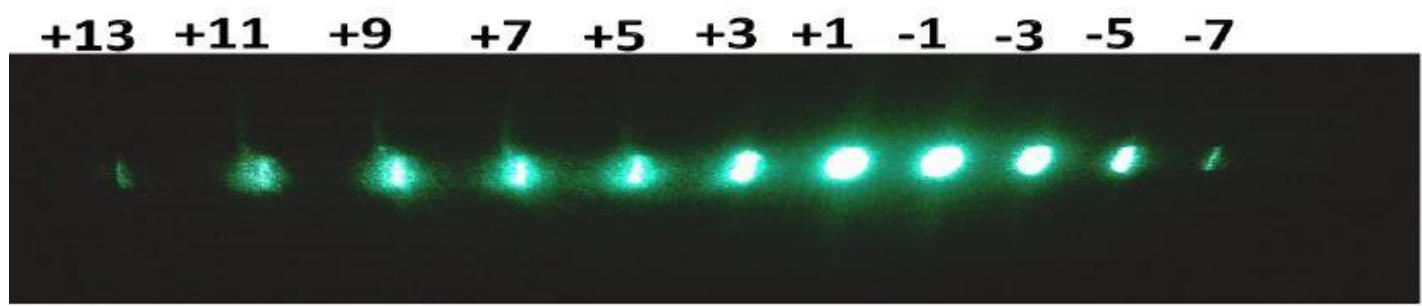

Figure 2.3: Nine non-Bragg orders seen during self-diffraction in PR LN [14].

\subsection{Derivation of Spatial Evolution of Bragg and Non-Bragg Orders for}

\section{Interacting Plane Waves}

As stated earlier the objective of this thesis is to study the spatial evolution of Bragg and non-Bragg orders in a PR material. First the interaction equations of Bragg and non-Bragg orders are derived for the case of two incident plane waves and considering the interactions of two Bragg and two non-Bragg orders in a PR material. The amplitudes and phases for the Bragg and non-Bragg orders can be found by first decomposing the total optical field inside the PR material as

$$
E_{p}(x, z)=\left[E_{1}(z) e^{-j\left(\frac{K}{2}\right) x}+E_{-1}(z) e^{j\left(\frac{K}{2}\right) x}+E_{3}(z) e^{-j 3\left(\frac{K}{2}\right) x}+E_{-3}(z) e^{j 3\left(\frac{K}{2}\right) x}\right] e^{-j k_{0} z}
$$

where $K$ is the spatial frequency of the Bragg grating (to be made more precise below), and substituting in the Helmholtz equation

$$
\nabla^{2} E_{p}+k^{2} E_{p}=0
$$

with

$$
k=n k_{0}=\left(n_{0}+\Delta n\right) k_{0}
$$

In Eq. (2.4), $k_{0}$ is the propagation constant and $n_{0}$ is the ambient refractive index which has been set to unity for simplicity. $\theta$ is the angle between the pump beam and probe beam. $x$ denotes the transverse coordinate and $z$ denotes the nominal direction of 
propagation. $E_{l}, l=-3,-1,1,3$ denote the optical fields for the $l^{\text {th }}$ order. The change in refractive index $\Delta n$ can be represented in terms of the intensity $I$, photovoltaic coefficient $c_{1}[11]$

$$
\Delta n=c_{1} I+c_{2} \frac{\partial I}{\partial x}
$$

once again using a component of the intensity gradient. It is noted that $\Delta n$ and $I$ can also be a slowly varying function of $z$.

Upon calculating the total intensity using Eq. (2.2) and substituting in Eq. (2.5), the change in refractive index can be determined. Then by rearranging the terms, the spatial evolution equations for the Bragg and non-Bragg orders can be derived. The evolution equations for the four interacting orders are given by

$$
\begin{gathered}
\frac{\partial E_{1}}{\partial z}=-j k_{0} E_{1}\left[c_{1}\left(\left|E_{1}\right|^{2}+2\left|E_{-1}\right|^{2}+2\left|E_{3}\right|^{2}+2\left|E_{-3}\right|^{2}\right)-\frac{1}{2}\left(\frac{K}{2 k_{0}}\right)^{2}\right]- \\
j k_{0} c_{1}\left[2 E_{3} E_{-1} E_{1}^{*}+2 E_{3} E_{-3} E_{-1}^{*}+2 E_{-1} E_{-1} E_{-3}^{*}\right]
\end{gathered}
$$

$$
\begin{gathered}
\frac{\partial E_{-1}}{\partial z}=-j k_{0} E_{-1}\left[c_{1}\left(2\left|E_{1}\right|^{2}+\left|E_{-1}\right|^{2}+2\left|E_{3}\right|^{2}+2\left|E_{-3}\right|^{2}\right)-\frac{1}{2}\left(\frac{K}{2 k_{0}}\right)^{2}\right]- \\
j k_{0} c_{1}\left[2 E_{-3} E_{1} E_{-1}^{*}+2 E_{3} E_{-3} E_{1}^{*}+2 E_{1} E_{1} E_{3}^{*}\right],
\end{gathered}
$$

$$
\begin{aligned}
& \frac{\partial E_{3}}{\partial z}=-j k_{0} E_{3}\left[c_{1}\left(2\left|E_{1}\right|^{2}+2\left|E_{-1}\right|^{2}+\left|E_{3}\right|^{2}+2\left|E_{-3}\right|^{2}\right)-\frac{9}{2}\left(\frac{K}{2 k_{0}}\right)^{2}\right]- \\
& j k_{0} c_{1}\left[2 E_{1} E_{-1} E_{-3}^{*}+2 E_{1} E_{1} E_{-1}^{*}\right] \\
& \frac{\partial E_{-3}}{\partial z}=-j k_{0} E_{-3}\left[c_{1}\left(2\left|E_{1}\right|^{2}+2\left|E_{-1}\right|^{2}+2\left|E_{3}\right|^{2}+\left|E_{-3}\right|^{2}\right)-\frac{9}{2}\left(\frac{K}{2 k_{0}}\right)^{2}\right]-
\end{aligned}
$$




$$
j k_{0} c_{1}\left[2 E_{1} E_{-1} E_{3}^{*}+2 E_{-1} E_{-1} E_{1}^{*}\right]
$$

where

$$
K=2 k_{0} \sin \left(\frac{\theta}{2}\right)
$$

In deriving Eqs. (2.4) - (2.7) the interactions between two Bragg and two non-Bragg orders are considered.

It is to be pointed out that for an arbitrary incident optical field from the object, $E_{-1}$ needs to be decomposed in terms of its angular plane wave spectral components. In this case, all interacting (Bragg and non-Bragg) orders also need to be decomposed into their respective spectra. This is discussed in detail in the next Section.

\subsection{Theoretical Model for Interacting Angular Spectra}

All of the calculations in the previous Section are based on the plane wave assumption for the incident as well as the Bragg and non-Bragg orders. In this Section the interaction equations of the Bragg and non-Bragg orders are modeled for the case of arbitrary incident beams which can be decomposed into their respective angular plane wave spectra.

By considering each diffracted order as a discrete collection of plane waves, the complex field for each order can be written as

$$
\begin{aligned}
& E_{1}=\sum_{i=-\infty}^{\infty} E_{1 i}^{\prime} e^{-j i \Delta K x}, \\
& E_{-1}=\sum_{i=-\infty}^{\infty} E_{-1 i}^{\prime} e^{+j i \Delta K x}, \\
& E_{3}=\sum_{i=-\infty}^{\infty} E_{3 i}^{\prime} e^{-j i \Delta K x}, \\
& E_{-3}=\sum_{i=-\infty}^{\infty} E_{-3 i}^{\prime} e^{+j i \Delta K x},
\end{aligned}
$$


where $E_{l}, l=-3,-1,1,3$ denote the total optical field for the $l^{\text {th }}$ order, $E_{l i}^{\prime}$ s denote the amplitude of the $i^{t h}$ angular plane wave component of the $l^{\text {th }}$ order, $\Delta K$ is the magnitude of the fundamental spatial frequency component in the angular plane wave spectrum profile (which is controllable by adjusting the repetition period of the spatial profile needed in the Fourier expansion implicit in the fast Fourier transform), $x$ denotes the transverse coordinate, and $z$ denotes the nominal direction of propagation. By replacing each diffracted order with its corresponding angular spectrum in the interaction equations (2.6a-d) of Bragg and non-Bragg orders, the coupled differential equations for every spectral component in each spectral order can be obtained. For instance, Eq. (2.6a) in terms of angular spectral components is written as

$$
\begin{gathered}
\left(\frac{\partial \sum_{i=-\infty}^{\infty} E_{1 i}^{\prime} e^{-j i k x}}{\partial \mathrm{z}}\right)=-j k_{0}\left[c _ { 1 } \left(\left|\sum_{i=-\infty}^{\infty} E_{1 i}^{\prime} e^{-j i k x}\right|^{2}+2\left|\sum_{i=-\infty}^{\infty} E_{-1 i}^{\prime} e^{+j i k x}\right|^{2}+\right.\right. \\
\left.\left.2\left|\sum_{i=-\infty}^{\infty} E_{3 i}^{\prime} e^{-j i k x}\right|^{2}+2\left|\sum_{i=-\infty}^{\infty} E_{-3 i}^{\prime} e^{+j i k x}\right|^{2}\right)-\frac{1}{2}\left(\frac{K+i k}{2 k_{0}}\right)^{2}\right] \sum_{i=-\infty}^{\infty} E_{1 i}^{\prime} e^{-j i k x}- \\
j k_{0} C_{1}\left[2 \sum_{i=-\infty}^{\infty} E_{3 i}^{\prime} e^{-j i k x} \sum_{i=-\infty}^{\infty} E_{-1 i}^{\prime} e^{+j i k x}\left(\sum_{i=-\infty}^{\infty} E_{1 i}^{\prime} e^{-j i k x}\right)^{*}+\right. \\
2 \sum_{i=-\infty}^{\infty} E_{3 i}^{\prime} e^{-j i k x} \sum_{i=-\infty}^{\infty} E_{-3 i}^{\prime} e^{+j i k x}\left(\sum_{i=-\infty}^{\infty} E_{-1 i}^{\prime} e^{+j i k x}\right)^{*}+ \\
\left.\left(\sum_{i=-\infty}^{\infty} E_{-1 i}^{\prime} e^{+j i k x}\right)^{2}\left(\sum_{i=-\infty}^{\infty} E_{-3 i}^{\prime} e^{+j i k x}\right)^{*}\right]
\end{gathered}
$$

A similar set of equations can be written for the other Bragg (-1) and non-Bragg $( \pm 3)$ orders. In Eq. (2.7) each term contains a triple sum which can be simplified using the Cauchy triple product for sum, viz., $\sum_{i} A_{i} \sum_{i} B_{i} \sum_{i} C_{i}=\sum_{i} \sum_{\beta} \sum_{\alpha} A_{\alpha} B_{\beta-\alpha} C_{i-\beta}$. Using this, the differential equations for the $i^{\text {th }}$ angular component for the +1 order becomes

$$
\begin{aligned}
\left(\frac{\partial E_{1 i}^{\prime}}{\partial z}\right)= & -j k_{0} c_{1}\left[\sum_{\beta} \sum_{\alpha} E_{1 \alpha}^{\prime} E_{1(\beta-\alpha)}^{\prime *} E_{1(i-\beta)}^{\prime}+2 \sum_{\beta} \sum_{\alpha} E_{-1 \alpha}^{\prime} E_{-1(\beta-\alpha)}^{\prime *} E_{1(i-\beta)}^{\prime}+\right. \\
& 2 \sum_{\beta} \sum_{\alpha} E_{3 \alpha}^{\prime} E_{3(\beta-\alpha)}^{\prime *} E_{1(i-\beta)}^{\prime}+2 \sum_{\beta} \sum_{\alpha} E_{-3 \alpha}^{\prime} E_{-3(\beta-\alpha)}^{\prime *} E_{1(i-\beta)}^{\prime}+
\end{aligned}
$$




$$
\begin{aligned}
& 2 \sum_{\beta} \sum_{\alpha} E_{3 \alpha}^{\prime} E_{-1(\beta-\alpha)}^{\prime} E_{1(i-\beta)}^{\prime *}+2 \sum_{\beta} \sum_{\alpha} E_{3 \alpha}^{\prime} E_{-3(\beta-\alpha)}^{\prime} E_{-1(i-\beta)}^{\prime *}+ \\
& \left.\sum_{\beta} \sum_{\alpha} E_{-1 \alpha}^{\prime} E_{-1(\beta-\alpha)}^{\prime} E_{-3(i-\beta)}^{\prime *}\right]-j k_{0} \frac{1}{2}\left(\frac{i k+K}{2 k_{0}}\right)^{2} E_{1 i}^{\prime}
\end{aligned}
$$

Similarly, the differential equations for the $i^{\text {th }}$ angular component for the -1 and \pm 3 orders become

$$
\begin{aligned}
& \left(\frac{\partial E_{-1 i}^{\prime}}{\partial z}\right)=-j k_{0} c_{1}\left[2 \sum_{\beta} \sum_{\alpha} E_{1 \alpha}^{\prime} E_{1(\beta-\alpha)}^{\prime *} E_{-1(i-\beta)}^{\prime}+\sum_{\beta} \sum_{\alpha} E_{-1 \alpha}^{\prime} E_{-1(\beta-\alpha)}^{\prime *} E_{-1(i-\beta)}^{\prime}+\right. \\
& 2 \sum_{\beta} \sum_{\alpha} E_{3 \alpha}^{\prime} E_{3(\beta-\alpha)}^{\prime *} E_{-1(i-\beta)}^{\prime}+2 \sum_{\beta} \sum_{\alpha} E_{-3 \alpha}^{\prime} E_{-3(\beta-\alpha)}^{\prime *} E_{-1(i-\beta)}^{\prime}+ \\
& 2 \sum_{\beta} \sum_{\alpha} E_{-3 \alpha}^{\prime} E_{1(\beta-\alpha)}^{\prime} E_{-1(i-\beta)}^{\prime *}+2 \sum_{\beta} \sum_{\alpha} E_{3 \alpha}^{\prime} E_{-3(\beta-\alpha)}^{\prime} E_{1(i-\beta)}^{\prime *}+ \\
& \left.\sum_{\beta} \sum_{\alpha} E_{1 \alpha}^{\prime} E_{1(\beta-\alpha)}^{\prime} E_{3(i-\beta)}^{\prime *}\right]-j k_{0} \frac{1}{2}\left(\frac{i k+K}{2 k_{0}}\right)^{2} E_{-1 i}^{\prime}, \\
& \left(\frac{\partial E_{3 i}^{\prime}}{\partial z}\right)=-j k_{0} c_{1}\left[2 \sum_{\beta} \sum_{\alpha} E_{1 \alpha}^{\prime} E_{1(\beta-\alpha)}^{\prime *} E_{3(i-\beta)}^{\prime}+2 \sum_{\beta} \sum_{\alpha} E_{-1 \alpha}^{\prime} E_{-1(\beta-\alpha)}^{\prime *} E_{3(i-\beta)}^{\prime}+\right. \\
& \sum_{\beta} \sum_{\alpha} E_{3 \alpha}^{\prime} E_{3(\beta-\alpha)}^{\prime *} E_{3(i-\beta)}^{\prime}+2 \sum_{\beta} \sum_{\alpha} E_{-3 \alpha}^{\prime} E_{-3(\beta-\alpha)}^{\prime *} E_{3(i-\beta)}^{\prime}+ \\
& \left.2 \sum_{\beta} \sum_{\alpha} E_{-1 \alpha}^{\prime} E_{1(\beta-\alpha)}^{\prime} E_{-3(i-\beta)}^{\prime *}+\sum_{\beta} \sum_{\alpha} E_{1 \alpha}^{\prime} E_{1(\beta-\alpha)}^{\prime} E_{-1(i-\beta)}^{\prime *}\right]- \\
& j k_{0} \frac{9}{2}\left(\frac{i k+K}{2 k_{0}}\right)^{2} E_{3 i}^{\prime} \\
& \left(\frac{\partial E_{-3 i}^{\prime}}{\partial z}\right)=-j k_{0} c_{1}\left[2 \sum_{\beta} \sum_{\alpha} E_{1 \alpha}^{\prime} E_{1(\beta-\alpha)}^{\prime *} E_{-3(i-\beta)}^{\prime}+2 \sum_{\beta} \sum_{\alpha} E_{-1 \alpha}^{\prime} E_{-1(\beta-\alpha)}^{\prime *} E_{-3(i-\beta)}^{\prime}+\right. \\
& \sum_{\beta} \sum_{\alpha} E_{3 \alpha}^{\prime} E_{3(\beta-\alpha)}^{\prime *} E_{-3(i-\beta)}^{\prime}+\sum_{\beta} \sum_{\alpha} E_{-3 \alpha}^{\prime} E_{-3(\beta-\alpha)}^{\prime *} E_{-3(i-\beta)}^{\prime}+ \\
& \left.2 \sum_{\beta} \sum_{\alpha} E_{-1 \alpha}^{\prime} E_{1(\beta-\alpha)}^{\prime} E_{3(i-\beta)}^{\prime *}+\sum_{\beta} \sum_{\alpha} E_{1 \alpha}^{\prime} E_{-1(\beta-\alpha)}^{\prime} E_{1(i-\beta)}^{\prime *}\right]- \\
& j k_{0} \frac{9}{2}\left(\frac{i k+K}{2 k_{0}}\right)^{2} E_{-3 i}^{\prime} .
\end{aligned}
$$

Eqs. (2.9a-d) can be numerically solved using Runge-Kutta methods (ode45 in MATLAB $^{\odot}$ ) to obtain the exact solutions for the complex fields, and hence, intensities of the Bragg and non-Bragg diffraction orders. A total of 35 harmonics are used to simulate each diffraction order. Numerical results are discussed in detail in the following Chapters. 


\subsection{Derivation of Photovoltaic Coefficient $\left(c_{1}\right)$ Using Kukhtarev Equations}

The spatial evolution of Bragg and non-Bragg orders depends on different material parameters such as the value of photovoltaic coefficient $\left(c_{1}\right)$, diffusive coefficient $\left(c_{2}\right)$ etc. The values of these coefficients can be calculated by using different material parameters [9]. In this Section starting from Kukhtarev equations derivation of the value of photovoltaic coefficient is performed. All the material parameters used in the calculation of photovoltaic coefficient are taken from Ref. [9]. Eqs. (2.10a-c) represent the Kukhtarev equations in the steady state:

$$
\begin{aligned}
& J=k \alpha I=e \mu n_{e} E_{s c}-e D_{s} \frac{\partial n_{e}}{\partial x}, \\
& N_{D}(s I+\beta)=\gamma_{R} N_{D}^{+} n_{e} \\
& \frac{\partial E_{s c}}{\partial x}=\frac{e}{\varepsilon_{s}}\left(N_{A}-N_{D}^{+}\right) .
\end{aligned}
$$

In Eqs. (2.10a-c) $\alpha$ is the absorption coefficient, $s$ is the ionization cross-section, $n_{e}$ is the free electron density, $N_{D}^{+}$is the ionized donor concentration, $J$ is current density, $k$ is photovoltaic constant, $E_{S C}$ is the electro-static space charge field, $N_{D}$ is the donor concentration, $N_{A}$ is the acceptor concentration, $\gamma_{R}$ is the recombination rate, $D_{S}$ is the diffusion constant, $\varepsilon_{s}$ is the static dielectric constant, $\mu$ is the carrier mobility, $\beta$ is the thermal excitation rate, $I$ is the intensity distribution of optical field, and $e$ is the charge of electron.

Using Eq. (2.10b), $n_{e}$ can be represented as

$$
n_{e}=\frac{(s I+\beta) N_{D}}{\gamma_{R} N_{D}^{+}} .
$$

From Eq. (2.10c), $N_{D}^{+}$can be written as

$$
N_{D}^{+}=N_{A}-\frac{\varepsilon_{s}}{e} \frac{\partial E_{s c}}{\partial x}
$$


Using Eq. (2.12), Eq. (2.11) can be rewritten as

$$
n_{e}=\frac{(s I+\beta) N_{D}}{\gamma_{R}\left(N_{A}-\frac{\varepsilon_{s}}{e} \frac{\partial E_{s C}}{\partial x}\right)}
$$

The spatial derivative of Eq. (2.10b) with respect to $x$ can be written as

$$
s N_{D} \frac{\partial I}{\partial x}=\gamma_{R} N_{D}^{+} \frac{\partial n_{e}}{\partial x}+\gamma_{R} n_{e} \frac{\partial N_{D}^{+}}{\partial x} .
$$

From Eq. (2.10a) and Eq. (2.10c), $\frac{\partial n_{e}}{\partial x}, \frac{\partial N_{D}^{+}}{\partial x}$ can be written as

$$
\begin{aligned}
& \frac{\partial n_{e}}{\partial x}=\left(\frac{\mu}{D_{s}} n_{e} E_{s c}-\frac{k \alpha I}{e D_{s}}\right), \\
& \frac{\partial N_{D}^{+}}{\partial x}=-\frac{\varepsilon_{s}}{e} \frac{\partial^{2} E_{s c}}{\partial x^{2}} .
\end{aligned}
$$

By substituting Eqs. (2.12), (2.13), (2.15), (2.16) in Eq. (2.14),

$$
\begin{aligned}
s N_{D} \frac{\partial I}{\partial x}= & \gamma_{R}\left(N_{A}-\frac{\varepsilon_{s}}{e} \frac{\partial E_{S c}}{\partial x}\right)\left[\frac{\mu}{D_{s}} \frac{(s I+\beta) N_{D}}{\gamma_{R}\left(N_{A}-\frac{\varepsilon_{S}}{e} \frac{\partial E_{S c}}{\partial x}\right)} E_{s c}-\frac{k \alpha I}{e D_{S}}\right]- \\
& \gamma_{R} \frac{(s I+\beta) N_{D}}{\gamma_{R}\left(N_{A}-\frac{\varepsilon_{S}}{e} \frac{\partial E_{S c}}{\partial x}\right)} \frac{\varepsilon_{S}}{e} \frac{\partial^{2} E_{s c}}{\partial x^{2}}
\end{aligned}
$$

By assuming $N_{A} \gg \frac{\varepsilon_{S}}{e} \frac{\partial E_{s c}}{\partial x}$ Eq. (2.17) reduces to

$$
s N_{D} \frac{\partial I}{\partial x}=\gamma_{R} N_{A}\left[\frac{\mu(s I+\beta) N_{D}}{D_{S} \gamma_{R} N_{A}} E_{S c}-\frac{k \alpha I}{e D_{S}}\right]-\frac{(s I+\beta) N_{D}}{N_{A}} \frac{\varepsilon_{S}}{e} \frac{\partial^{2} E_{S c}}{\partial x^{2}} .
$$

Upon simplification, Eq. (2.18) becomes

$$
s N_{D} \frac{\partial I}{\partial x}+\frac{k \alpha \gamma_{R} N_{A}}{e D_{S}} I=\frac{(s I+\beta) N_{D}}{N_{A}}\left[\frac{\mu N_{A} E_{S C}}{D_{S}}-\frac{\varepsilon_{S}}{e} \frac{\partial^{2} E_{s c}}{\partial x^{2}}\right] .
$$

Assuming $\frac{\mu N_{A} E_{S c}}{D_{S}} \gg \frac{\varepsilon_{S}}{e} \frac{\partial^{2} E_{S c}}{\partial x^{2}}$ Eq. (2.19) reduces to

$$
E_{S C}=\frac{D_{S}}{\mu N_{D}(s I+\beta)}\left(\frac{k \alpha \gamma_{R} N_{A}}{e D_{S}} I+s N_{D} \frac{\partial I}{\partial x}\right)
$$


By rearranging terms in Eq. (2.20),

$$
E_{S C}=\frac{k \alpha \gamma_{R} N_{A}}{\mu e N_{D}(s I+\beta)} I+\frac{s D_{S}}{\mu(s I+\beta)} \frac{\partial I}{\partial x} \equiv E_{P V}+E_{D I F F}
$$

where $E_{P V}$ is the photovoltaic contribution to the space charge field, and $E_{D I F F}$ represents the contribution from carrier diffusion. Now the change in refractive index $\Delta n$ can be written as

$$
\Delta n=-\frac{1}{2} n_{o}^{3} r_{33} E_{s c}
$$

In Eq. (2.22), $r_{33}$ is the electro-optic coefficient, and $n_{o}$ is the refractive index of LN (assumed ordinary here as an example). By substituting Eq. (2.21) into Eq. (2.22),

$$
\Delta n=-\frac{1}{2} n_{o}^{3} r_{33} \frac{k \alpha \gamma_{R} N_{A}}{\mu e N_{D}(s I+\beta)} I-\frac{1}{2} n_{o}^{3} r_{33} \frac{s D_{S}}{\mu(s I+\beta)} \frac{\partial I}{\partial x} .
$$

Comparing Eq. (2.23) with Eq. (2.1),

$$
\begin{aligned}
& c_{1}=-\frac{1}{2} n_{o}^{3} r_{33} \frac{k \alpha \gamma_{R} N_{A}}{\mu e N_{D}(s I+\beta)} \mathrm{m}^{2} / \mathrm{W}, \\
& c_{2}=-\frac{1}{2} n_{o}^{3} r_{33} \frac{s D_{s}}{\mu(s I+\beta)} \mathrm{m}^{3} / \mathrm{W} .
\end{aligned}
$$

In this work, the contribution of diffusion, which is small compared to the photovoltaic contribution in PR LN, has been neglected. An estimate of $c_{1}$ can be found by substituting the various material parameters in Eq. (2.24). For instance, choosing

$$
\begin{aligned}
& \frac{N_{A}}{N_{D}}=\frac{1}{2}, \gamma_{R}=10^{-15} \mathrm{~m}^{3} / \mathrm{s}, k=3 \cdot 10^{-11} \mathrm{~A} \cdot \mathrm{m} / \mathrm{W}, \alpha=130 \mathrm{~m}^{-1}, \mu=10^{-6} \mathrm{~m}^{2} / \mathrm{V} . \mathrm{s}, \\
& \beta=1 \mathrm{~s}^{-1}, e=-1.6 \cdot 10^{-19} \mathrm{C}, r_{33}=30.8 \mathrm{pm} / \mathrm{V}, n_{o}=2.22, s=6 \cdot 10^{-5} \mathrm{~m}^{2} / \mathrm{J},
\end{aligned}
$$

and assuming $s I \ll \beta$,

$$
c_{1}=-2.1 \cdot 10^{-11} \mathrm{~m}^{2} / \mathrm{W}
$$


All the material parameters which are used in the calculation of photovoltaic coefficient are taken from Ref. [9].

To study the spatial evolution of Bragg and non-Bragg orders, Eqs. (2.9a-d) should be numerically solved using Runge-Kutta methods (ode45 in MATLAB ${ }^{\odot}$ ) to obtain the exact solutions for the complex fields. While performing the simulations the value of $c_{1}$ shown in Eq. (2.26) is used for the photovoltaic coefficient. Also the powers of the incident beam profiles are chosen in such a way that the condition $s I \ll \beta$ is satisfied. This is because the value of $c_{1}$ holds good only if the condition $s I \ll \beta$ is satisfied.

\subsection{Conclusion}

In this Chapter, the PR effect has been discussed in some detail. An experiment demonstrating Bragg and non-Bragg orders in PR LN is described. The evolution equations of Bragg and non-Bragg orders are derived for the case of plane waves, and extended for the case of arbitrary interacting optical fields using the angular plane wave spectral approach. The expression and a typical value for the photovoltaic coefficient is also derived. In the following Chapters, the exact solutions of the interaction equations of the Bragg and non-Bragg orders are obtained for a variety of beam profiles. 


\section{CHAPTER III}

\section{SPECTRAL ANALYSIS OF BRAGG AND NON-BRAGG ORDERS USING GAUSSIAN BEAM PROFILES}

\subsection{Introduction}

In Chapter 2 a theoretical model has been developed in detail to study the interactions of Bragg and non-Bragg orders in a PR material using the angular spectrum approach. A set of coupled partial differential equations (Eqs. (2.9a-d)) are derived by considering the photovoltaic effect as the dominant contributor to the PR effect, as is the case in PR LN, but can be readily extended to include the effect of diffusion. The evolution of Bragg and non-Bragg orders in a PR material depends on different incident beam parameters such as beam powers, waists and angle between the incident beams along with material parameters.

In this Chapter, Eqs. (2.9a-d) are numerically solved in MATLAB ${ }^{\odot}$ for different incident Gaussian beam profiles. A detailed numerical analysis is performed by varying all the incident beam parameters to observe the spatial evolution of Bragg and nonBragg orders in the PR material.

\subsection{Bragg and Non-Bragg Orders for Identical Incident Gaussian Beam Profiles}

As stated earlier, the spatial evolution of Bragg and non-Bragg orders depends on different incident beam parameters such as incident beam powers, beam waists and the angle between the two incident beams. In this Section, Eqs. (2.9a-d) are numerically solved in MATLAB ${ }^{\odot}$ using Range-Kutta methods to demonstrate the spatial evolution 
of Bragg and non-Bragg orders in PR LN by keeping the incident beam powers and incident beam waists and angle between them constant. $P_{1}, W_{1}$ represent the power and waist of pump (reference) beam or first Bragg order, respectively, and $P_{-1}, W_{-1}$ represent the power and waist of probe (object) beam or second Bragg order, respectively. $\theta$ is the external angle between the incident beams. The thickness of the crystal $L$ is taken as $1 \mathrm{~mm}$. Experimentally while writing the hologram in a PR material, the pump beam and probe beam should be interfered in such a way that the interference pattern should be created at the center plane of the PR crystal.

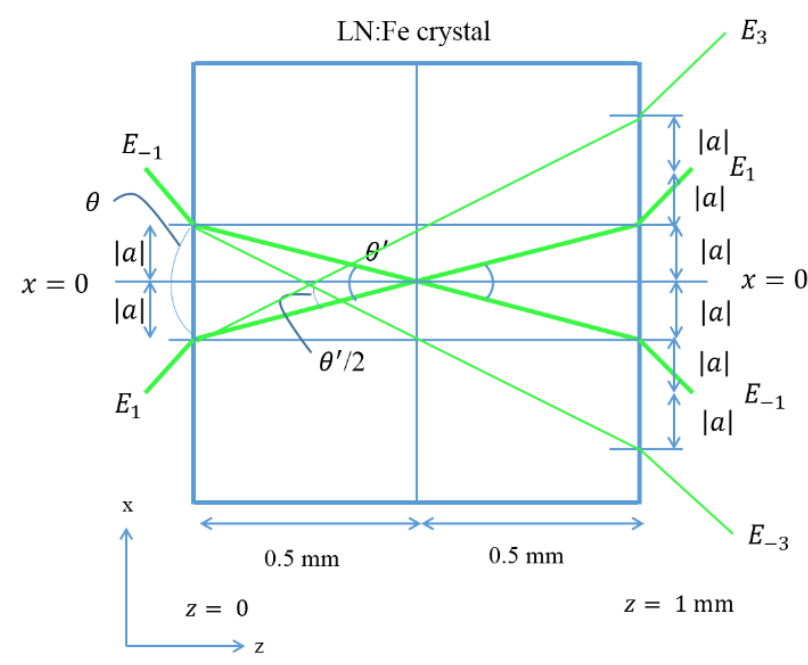

Figure 3.1: Geometrical representation of Bragg and non-Bragg orders propagating in PR LN.

Figure 3.1 illustrates the geometrical representation of the Bragg and non-Bragg orders propagating in PR LN. The Bragg orders are interfered at the center plane of crystal, i.e. at $z=0.5 \mathrm{~mm}$. In Fig $3.1 \theta^{\prime}$ is the internal angle between the pump beam and probe beam. For convenience, the plane at $z=0$ can be called as entrance plane of PR LN crystal and the plane at $z=1 \mathrm{~mm}$ can be called as exit plane of PR LN crystal. The amount of shift to be introduced numerically between pump beam and probe beam at the entrance plane depends on the plane they interfere and the internal angle between the incident beams. To make the pump beam and probe beam interact 
at the center plane of the crystal, i.e. at $z=0.5 \mathrm{~mm}$ numerically, a shift of $|a|$ to both the pump and probe beams has been introduced at the entrance plane of the crystal. The value of $|a|$ can be calculated geometrically from Fig 3.1. $|a|$ depends on the internal angle between the Bragg orders. At the exit plane, the amount of shift in the peak values of Bragg and non-Bragg orders from $x=0$ can be calculated geometrically from Figure 3.1. From $x=0$ the peak value of the first Bragg order $(+1)$ should have a shift of $|a|$ at the exit plane. Also, from $x=0$ the peak value of first non-Bragg order $(+3)$ should have a shift of $3|a|$ at exit plane. Similar arguments can be made for the -1 and -3 orders. If $\theta$ is the external angle between the incident beams then from law of refraction the internal angle $\theta^{\prime}$ can be approximately taken as the ratio of external angle to the refractive index of the PR LN, which is taken as 2.22. In this Section, numerical analysis is performed by taking the incident beam parameters identical.

3.2.1 Numerical results for $P_{1}=P_{-1}=20 \mathrm{~mW} ; W_{1}=W_{-1}=0.4 \mathrm{~mm} ; \theta=0.5^{\circ}$ In this Section, Eqs. (2.9a-d) are numerically solved in MATLAB ${ }^{\odot}$ for two identical Gaussian beam profiles whose powers are $20 \mathrm{~mW}$ and waists are $0.4 \mathrm{~mm}$ to study the spatial evolution of Bragg and non-Bragg orders. The power of the incident beams should be chosen in such a way that the condition $s I \ll \beta$ is satisfied. With the external angle between the incident beams set at $0.5^{\circ}$, the internal angle is $0.225^{\circ}$. From Figure 3.1 the value of $|a|$ can be found to be

$$
|a|=0.5 \tan \left(\frac{0.225}{2}\right) \mathrm{mm} \approx 1 \mu \mathrm{m}
$$

Therefore, a shift of $a=-1 \mu \mathrm{m}$ should be introduced to the pump beam. In other words the pump beam is not centered at $x=0$. The center of pump beam is shifted by $-1 \mu \mathrm{m}$ from $x=0$. Similarly a shift of $a=+1 \mu \mathrm{m}$ should be introduced to probe 
beam. Figure 3.2(a, b) show the incident Gaussian beam profiles of the pump (reference) and probe (object) beams.

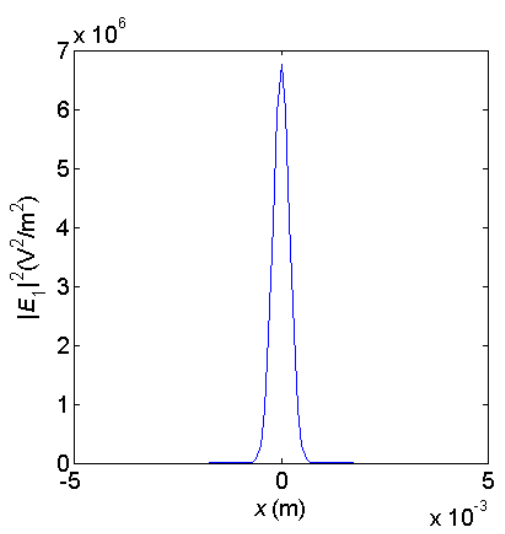

(a)

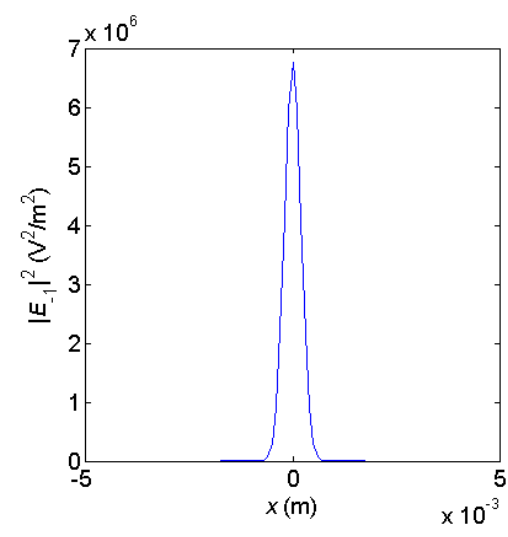

(b)

Figure 3.2: Gaussian beam profiles of incident (a) pump (reference) beam and (b) probe (object) beams. $P_{1}=20 \mathrm{~mW}, W_{1}=0.4 \mathrm{~mm} ; P_{-1}=20 \mathrm{~mW}, W_{-1}=0.4 \mathrm{~mm}$. The spatial shifts of $1 \mu \mathrm{m}$ is not discernible at this scale.

The pump (reference) and probe (object) are used as incident beams to solve Eqs. (2.9a-d) in MATLAB $^{\odot}$ to study the spatial evolution of Bragg and non-Bragg orders. Figures 3.3(a)-(d) illustrate the spatial evolution of Bragg and non-Bragg orders in PR LN for the case where $P_{1}=P_{-1}=20 \mathrm{~mW} ; W_{1}=W_{-1}=0.4 \mathrm{~mm} ; \theta=$ $0.5^{\circ}$ 


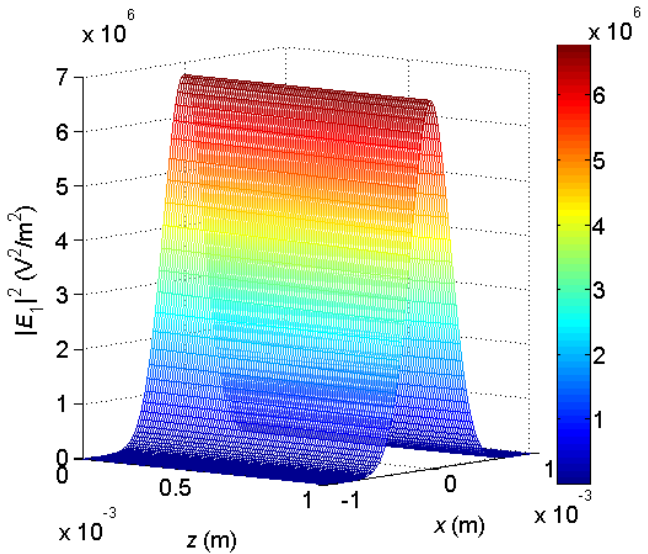

(a)

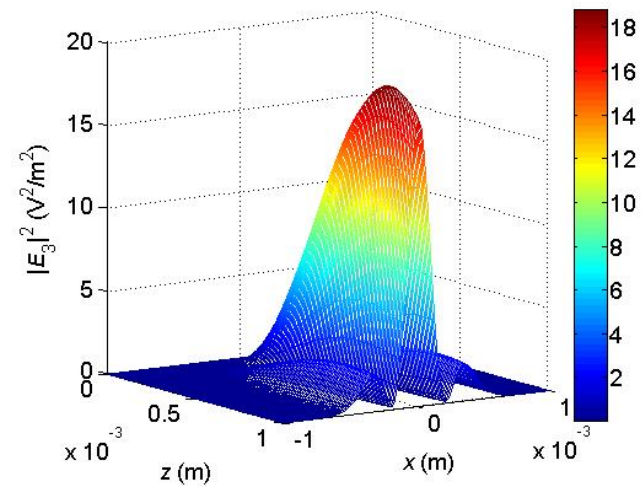

(c)

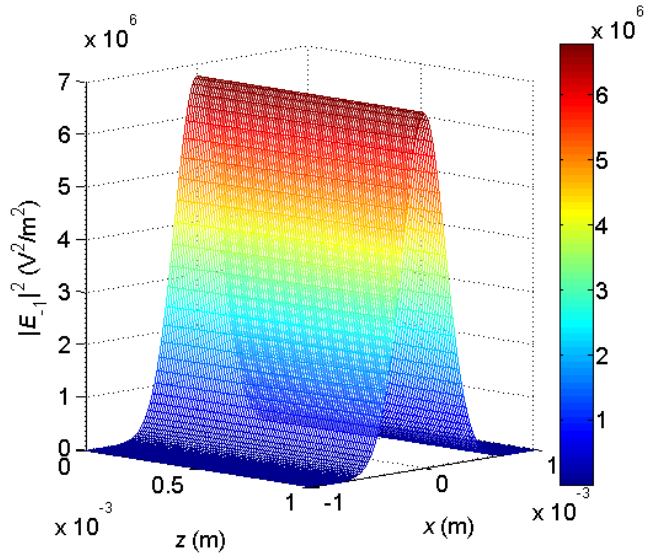

(b)

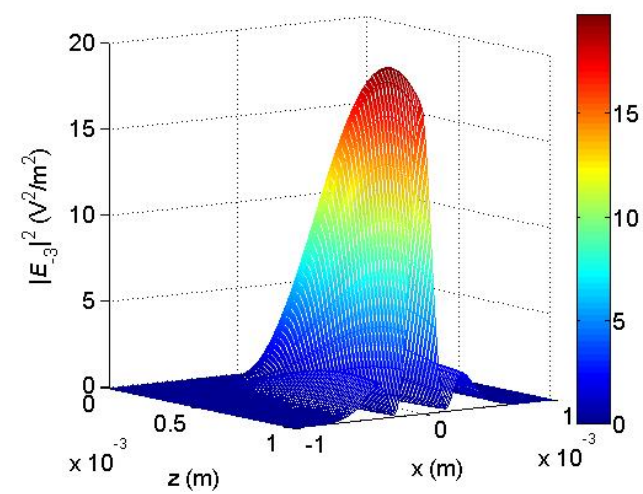

(d)

Figure 3.3: Spatial evolution of (a) first Bragg order $\left(E_{1}\right)$, (b) second Bragg order $\left(E_{-1}\right)$, (c) first non-Bragg order $\left(E_{3}\right)$, and (d) second non-Bragg order $\left(E_{-3}\right)$ in PR LN. $P_{1}=$ $20 \mathrm{~mW}, W_{1}=0.4 \mathrm{~mm} ; P_{-1}=20 \mathrm{~mW}, W_{-1}=0.4 \mathrm{~mm}$. 


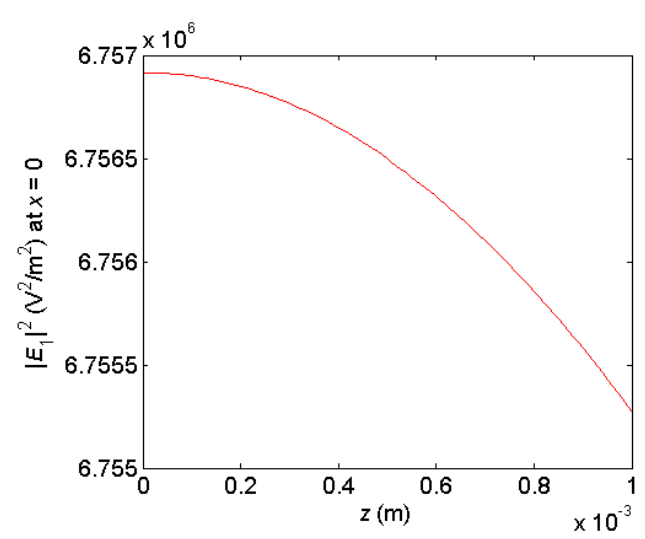

(a)

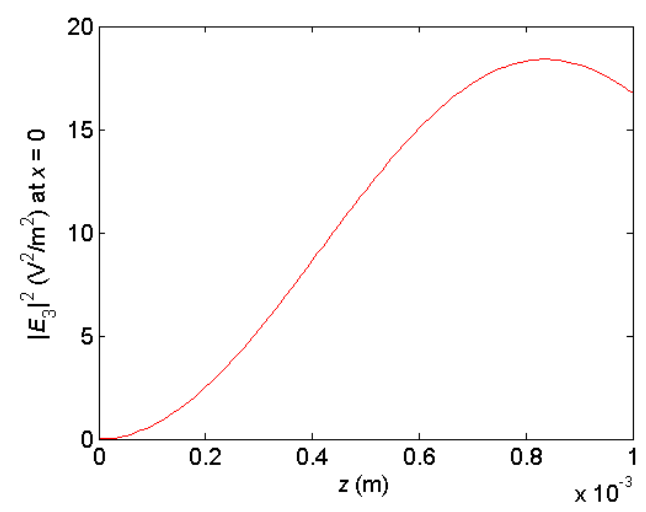

(c)

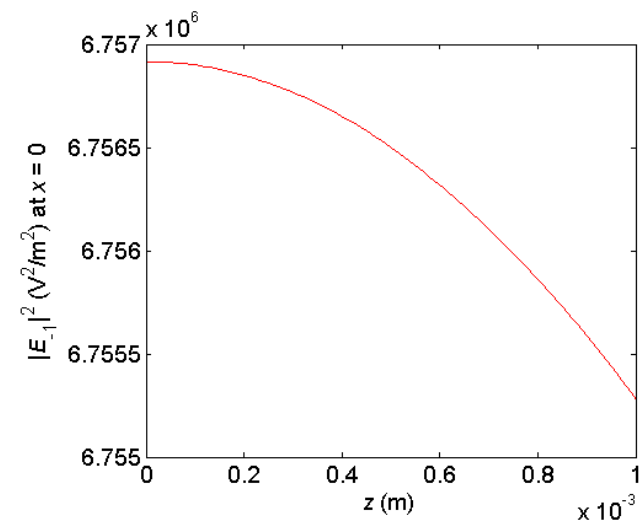

(b)

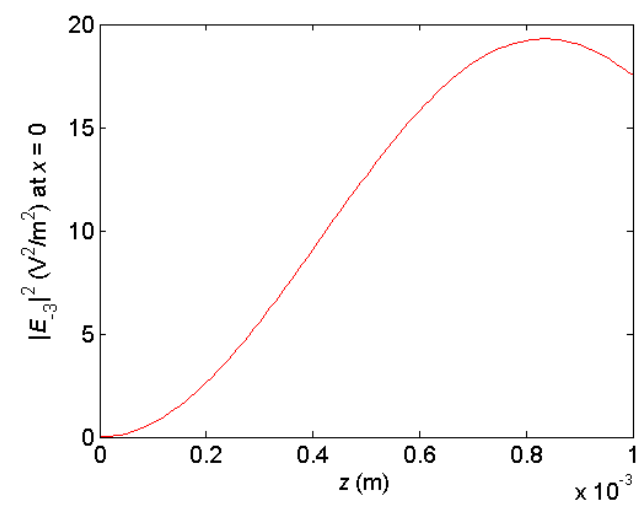

(d)

Figure 3.4: Variation of on-axis $(x=0)$ intensity of (a) first Bragg order $\left(E_{1}\right)$, (b) second Bragg order $\left(E_{-1}\right)$, (c) first non-Bragg order $\left(E_{3}\right)$, and (d) second non-Bragg order $\left(E_{-3}\right)$ during propagation. $P_{1}=20 \mathrm{~mW}, W_{1}=0.4 \mathrm{~mm} ; P_{-1}=20 \mathrm{~mW}, W_{-1}=$ $0.4 \mathrm{~mm}$.

From Figures 3.5(a)-(d) it is evident that as the incident beams propagate along PR LN the on-axis intensities of $E_{1}$ and $E_{-1}$ orders decrease from their initial value while the on-axis intensities of $E_{3}$ and $E_{-3}$ orders increases gradually from zero. The higher or non-Bragg orders are generated at the expense of energy from +1 and -1 (Bragg) orders. Also for identical incident profiles for the +1 and -1 orders, the profiles and hence on-axis intensities of the +3 and -3 (non-Bragg) orders are identical. The dependence of the non-Bragg orders on different incident +1 and -1 order on-axis intensities and widths are investigated below. 
A brief note on the spatial shift of the beams: there is a $2|a|=2 \mu \mathrm{m}$ shift for the first Bragg order between the entry and exit faces of the PR LN crystal (see Fig. 3.6(a)), and similarly for the second Bragg order; while the first and second non-Bragg orders are shifted $\pm 3|a|=3 \mu \mathrm{m}$ from $z=0$ at the exit plane (see Fig. 3.6(b) for the second non-Bragg order), as expected.

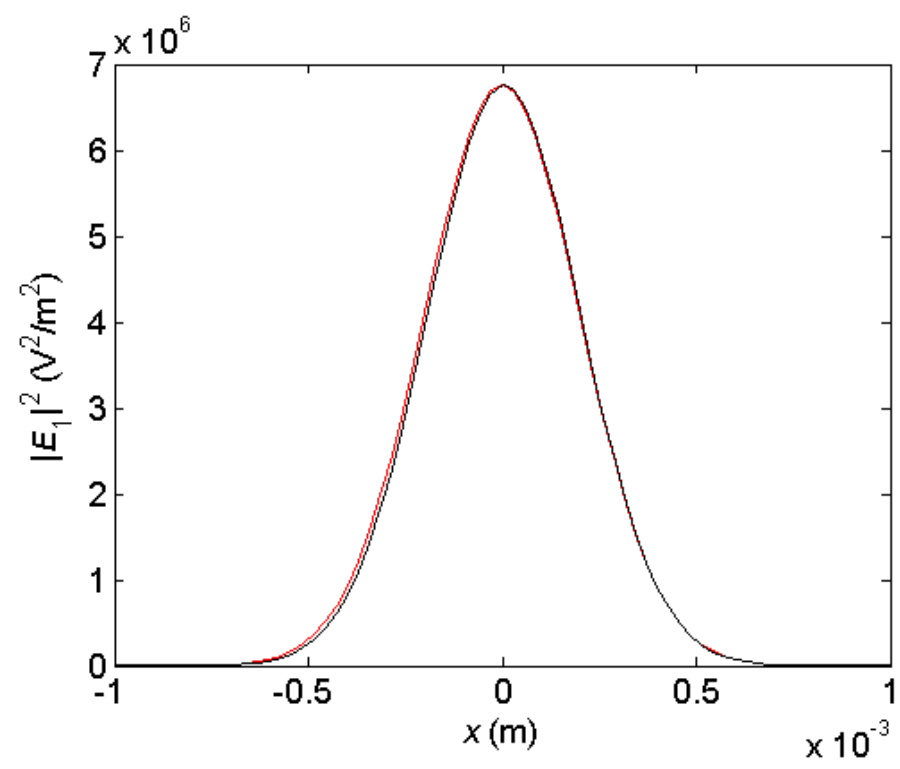

(a)

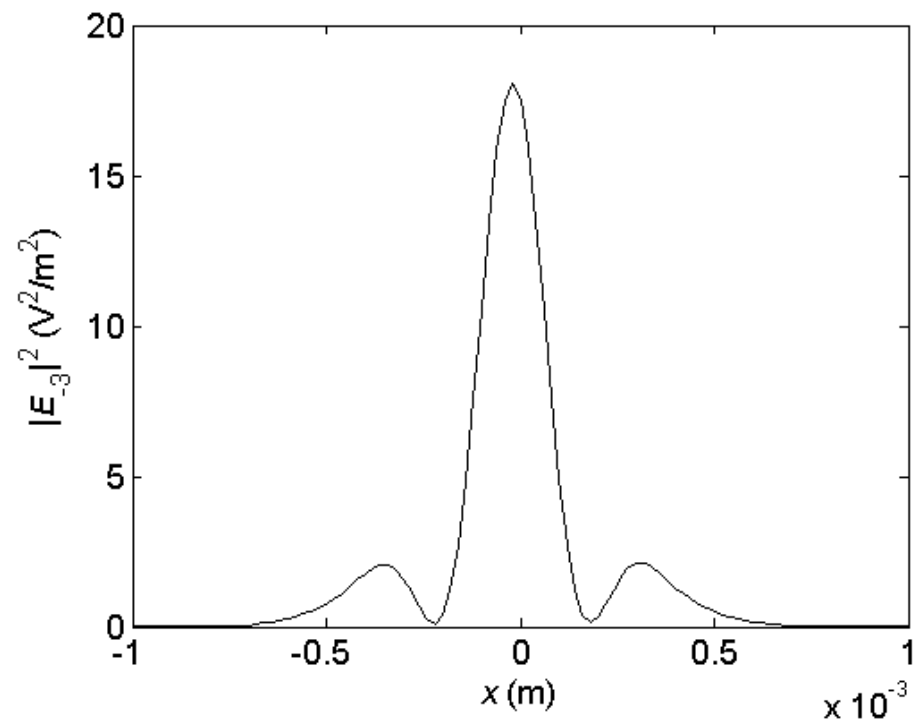

(b)

Figure 3.5: (a) First Bragg order $\left(E_{1}\right)$ at the entrance plane (red) and exit plane (black) of PR LN; (b) second non-Bragg order $\left(E_{-3}\right)$ at exit plane. 
As shown in Figures 3.3, the shape of the Bragg orders remain Gaussian through interaction in the PR material. The non-Bragg orders start evolving from the moment the Bragg orders start interacting with the PR material. The shape of the non-Bragg $\left(E_{3}, E_{-3}\right)$ orders do not remain Gaussian through their interaction but develop two side lobes. The pattern of the evolution of higher orders resembles that of a Gaussian beam when it is changing its mode. The precise reason for this is under investigation.

From Figure 2.2 it is evident that experimentally it is difficult to differentiate and observe the beam profiles of Bragg and non-Bragg orders exactly at the exit plane of the crystal of thickness $1 \mathrm{~mm}$. Imaging the exit plane will be pursued in the future. However, it is easier to monitor the Bragg and non-Bragg orders after propagation through some distance. Therefore, in Figures 3.6 (a)-(d), the far field pattern of the Bragg and non-Bragg orders have been computed for future comparison with experimental observations. While the Bragg orders resemble Gaussians, the non-Brag orders show spatial sidebands after a propagation distance of $50 \mathrm{~cm}$ due to the effect of mode conversion during interaction in the PR material.

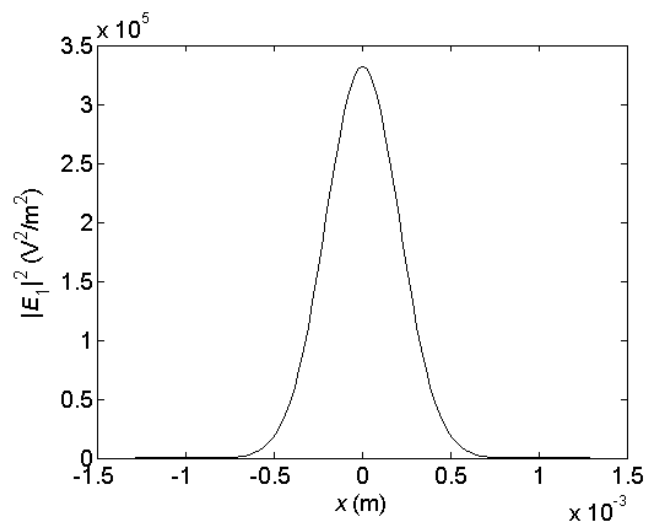

(a)

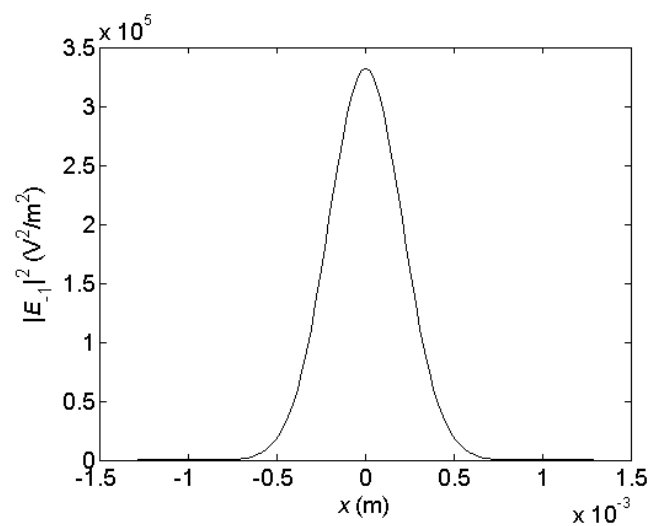

(b) 


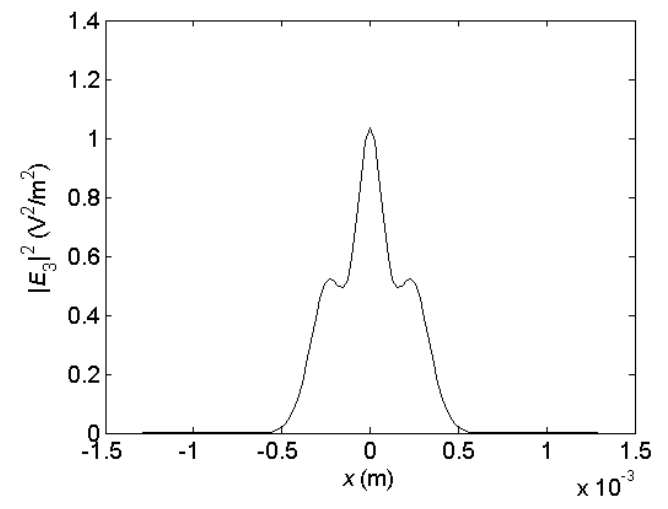

(c)

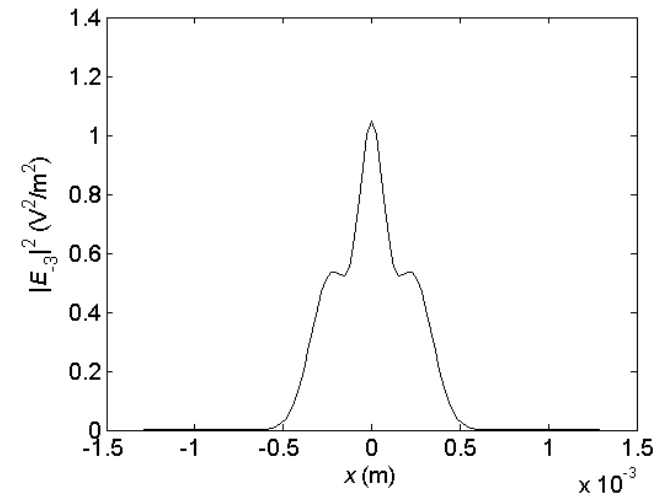

(d)

Figure 3.6: Far field pattern of the (a) first Bragg order $\left(E_{1}\right)$, (b) second Bragg order $\left(E_{-1}\right),(\mathrm{c})$ first non-Bragg order $\left(E_{3}\right)$, and (d) second non-Bragg order $\left(E_{-3}\right)$ after propagating a distance of $50 \mathrm{~cm}$ from exit plane of PR LN crystal.

\subsection{Bragg and Non-Bragg Orders for Incident Gaussian Beam Profiles with Different Powers and Identical Waists}

As stated earlier, the spatial evolution of Bragg and non-Bragg orders depends on different incident beam parameters such as incident beam powers, beam waists and the angle between the two incident beams along with material parameters. From Section 3.2 it is obvious that with identical incident Gaussian beam profiles higher orders show a strong evidence of mode conversion. To understand this phenomena much more numerical analysis is required. Numerical analysis can be performed by varying all the incident beam parameters. In this Section, numerical analysis is performed by varying the incident beam powers and keeping the incident beam waists equal. Numerical simulations are performed for two different cases:

1) $P_{1}=20 \mathrm{~mW}, P_{-1}=10 \mathrm{~mW} ; W_{1}=W_{-1}=0.4 \mathrm{~mm} ; \theta=0.5^{\circ}$.

2) $P_{1}=20 \mathrm{~mW}, P_{-1}=2 \mathrm{~mW} ; W_{1}=W_{-1}=0.4 \mathrm{~mm} ; \theta=0.5^{\circ}$. 
Numerical results for both the cases are discussed in subsequent Sections. For both the cases as the external angle between the incident beams is $0.5^{\circ}$, a shift of $a=-1 \mu \mathrm{m}$ and a shift of $a=+1 \mu \mathrm{m}$ has been introduced to pump and probe beams respectively.

\subsubsection{Numerical results for $P_{1}=20 \mathrm{~mW}, P_{-1}=10 \mathrm{~mW} ; W_{1}=W_{-1}=$} $0.4 \mathrm{~mm} ; \theta=0.5^{\circ}$

In this Section Eqs. (2.9a-d) are numerically solved in MATLAB ${ }^{\odot}$ for two Gaussian beam profiles having different powers. Once again, the powers of the incident beams should be chosen in such a way that the condition $s I \ll \beta$ is satisfied. The powers of pump $P_{1}$ and probe $P_{-1}$ beams are taken as $20 \mathrm{~mW}$ and $10 \mathrm{~mW}$ respectively and the waists of pump $W_{1}$ and probe $W_{-1}$ beams are taken as $0.4 \mathrm{~mm}$ respectively. Figure 3.7 $(a, b)$ show the incident Gaussian beam profiles of the pump (reference) and probe (object) beams.

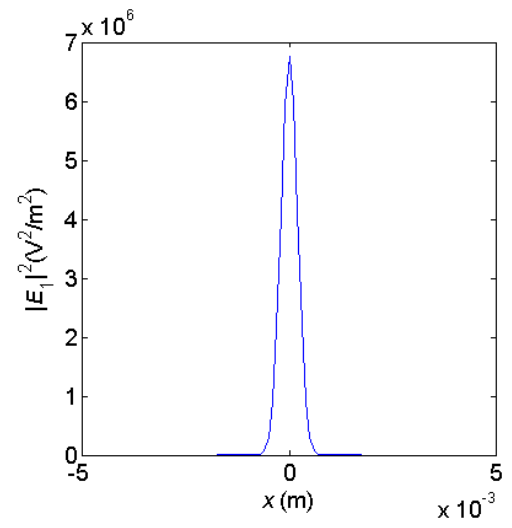

(a)

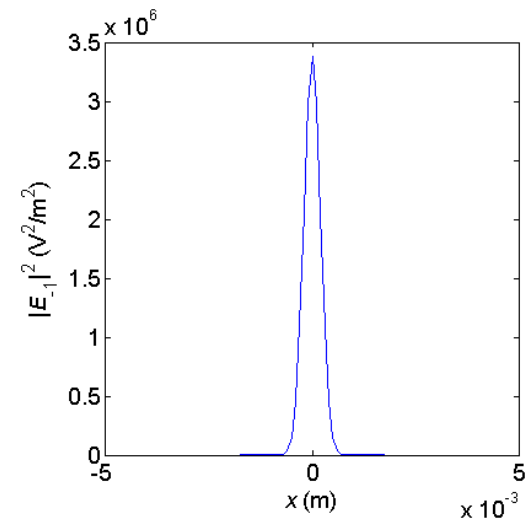

(b)

Figure 3.7: Gaussian beam profiles of incident (a) pump (reference) and (b) probe (object) beams. $P_{1}=20 \mathrm{~mW}, W_{1}=0.4 \mathrm{~mm} ; P_{-1}=10 \mathrm{~mW}, W_{-1}=0.4 \mathrm{~mm}$. The spatial shifts of $1 \mu \mathrm{m}$ is not discernible at this scale.

The pump and probe beams are used as incident Bragg orders to solve Eqs. (2.9a-d) in MATLAB $^{\odot}$ to study the spatial evolution of Bragg and non-Bragg orders. Figure 3.8(a)-(d) illustrate the spatial evolution of Bragg and non-Bragg orders in PR LN for the case where $P_{1}=20 \mathrm{~mW}, P_{-1}=10 \mathrm{~mW} ; W_{1}=W_{-1}=0.4 \mathrm{~mm} ; \theta=0.5^{\circ}$. 


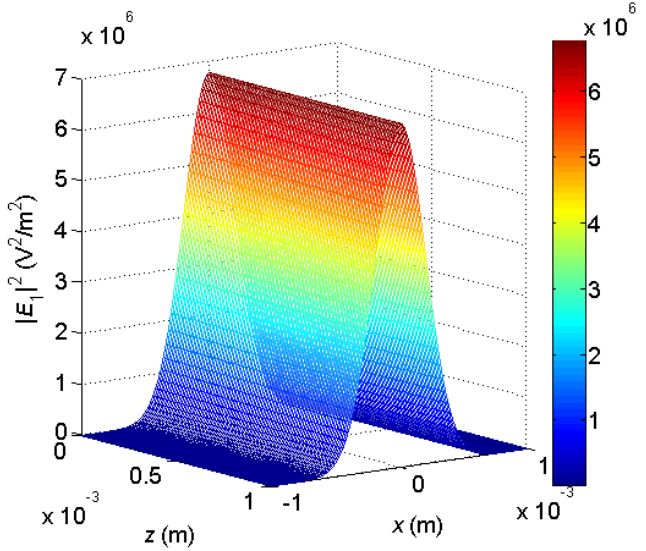

(a)

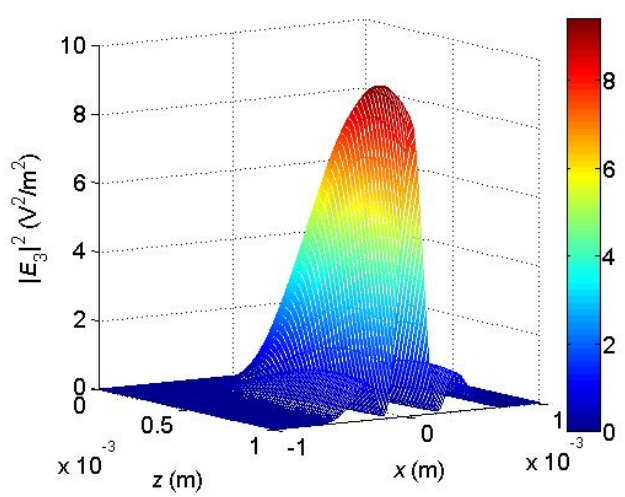

(c)

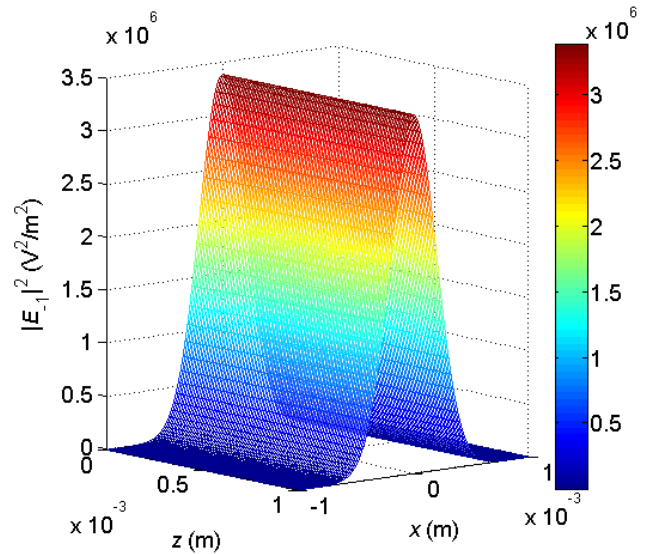

(b)

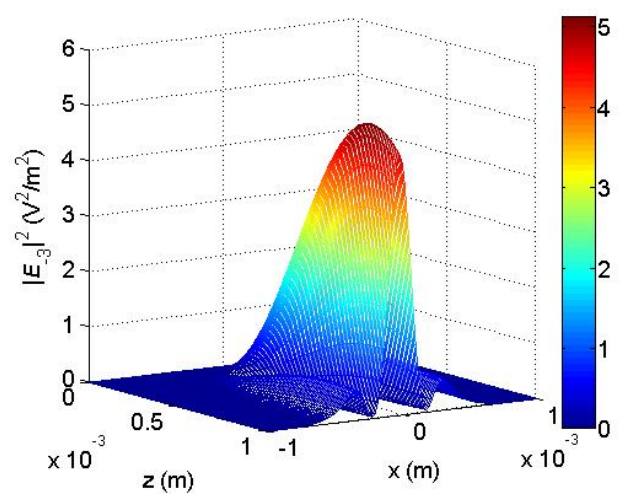

(d)

Figure 3.8: Spatial evolution of (a) first Bragg order $\left(E_{1}\right)$, (b) second Bragg order $\left(E_{-1}\right)$, (c) first non-Bragg order $\left(E_{3}\right)$, and (d) second non-Bragg order $\left(E_{-3}\right)$ in PR LN. $P_{1}=20 \mathrm{~mW}, W_{1}=0.4 \mathrm{~mm} ; P_{-1}=10 \mathrm{~mW}, W_{-1}=0.4 \mathrm{~mm}$.

While the qualitative variations of the Bragg and non-Bragg orders are the same as the case with identical incident beams, it is important to observe that when the power of the second Bragg order $\left(\left|E_{-1}\right|^{2}\right)$ is half of the power of first Bragg order $\left(\left|E_{1}\right|^{2}\right)$, the peak intensity of the second non-Bragg order $\left(\left|E_{-3}\right|^{2}\right)$ is also half of the peak intensity of the first non-Bragg order $\left(\left|E_{3}\right|^{2}\right)$.

This observation can also be verified theoretically by simple plane wave calculation from Eqs. (2.6c), (2.6d). From Eq. (2.6c) it is evident that the dominant term responsible for the evolution of first non-Bragg $\left(E_{3}\right)$ order is $E_{1} E_{1} E_{-1}^{*}$. 
In other words

$$
E_{3} \approx E_{1} E_{1} E_{-1}^{*}
$$

Similarly from Eq. (2.7c) it is evident that the dominant term responsible for the evolution of second non-Bragg $\left(E_{-3}\right)$ order is $E_{-1} E_{-1} E_{1}^{*}$. This means

$$
E_{-3} \approx E_{-1} E_{-1} E_{1}^{*}
$$

When $\left|E_{1}\right|^{2}=2\left|E_{-1}\right|^{2},\left|E_{3}\right|^{2}=2\left|E_{-3}\right|^{2}$. Therefore numerically and theoretically it can be concluded that the power of second non-Bragg order is reduced with reduction in the power of second Bragg order. Also from Figure 3.8(c)-(d) it can be observed that there is an evidence of mode conversion of incident beams as the higher orders evolve. In general, when $\left|E_{1}\right|^{2}=m\left|E_{-1}\right|^{2}$, it is expected that $\left|E_{3}\right|^{2}=m\left|E_{-3}\right|^{2}$, as is verified below.

\subsubsection{Numerical results for $P_{1}=20 \mathrm{~mW}, P_{-1}=2 \mathrm{~mW} ; W_{1}=W_{-1}=$ $0.4 \mathrm{~mm} ; \theta=0.5^{\circ}$}

Here the powers of pump $P_{1}$ and probe $P_{-1}$ beams are taken as $20 \mathrm{~mW}$ and $2 \mathrm{~mW}$ respectively and the waists of pump $W_{1}$ and probe $W_{-1}$ beams are taken as $0.4 \mathrm{~mm}$ respectively. Figure 3.9 $(\mathrm{a}, \mathrm{b})$ show the incident Gaussian beam profiles of the pump (reference) and probe (object) beams. 


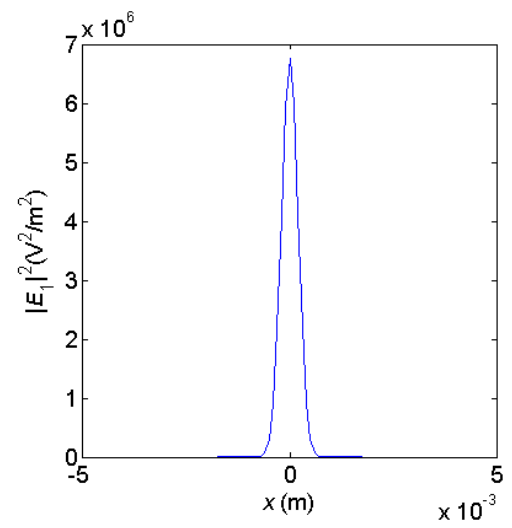

(a)

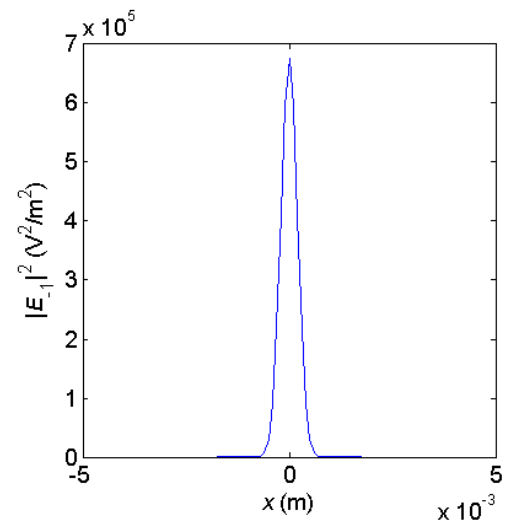

(b)

Figure 3.9: Gaussian beam profiles of incident (a) pump (reference) and (b) probe (object) beams. $P_{1}=20 \mathrm{~mW}, W_{1}=0.4 \mathrm{~mm} ; P_{-1}=2 \mathrm{~mW}, W_{-1}=0.4 \mathrm{~mm}$. The spatial shifts of $1 \mu \mathrm{m}$ is not discernible at this scale.

Once again, the pump (reference) and probe (object) are used as incident beams to solve Eqs. (2.9a-d) in MATLAB ${ }^{\odot}$ to study the spatial evolution of Bragg and nonBragg orders. Figure 3.10(a)-(d) illustrate the spatial evolution of Bragg and non-Bragg orders in PR LN for the case where $P_{1}=20 \mathrm{~mW}, P_{-1}=2 \mathrm{~mW} ; W_{1}=W_{-1}=$ $0.4 \mathrm{~mm} ; \theta=0.5^{\circ}$. As expected, when the power of probe beam or second Bragg order is reduced by $90 \%$ (or by a factor of 10) compared to that of pump beam or first Bragg order, it can be observed that the power of second non-Bragg order is also reduced by $90 \%$ (or by a factor of 10) compared to the power of first non-Bragg order (see Figure $3.10(\mathrm{c})-(\mathrm{d}))$.

The simulations in this sub-section are important since, in general, the probe power is almost always considerably less than the pump power. In the following Section numerical analysis is performed by varying the waists of incident beam profiles. 


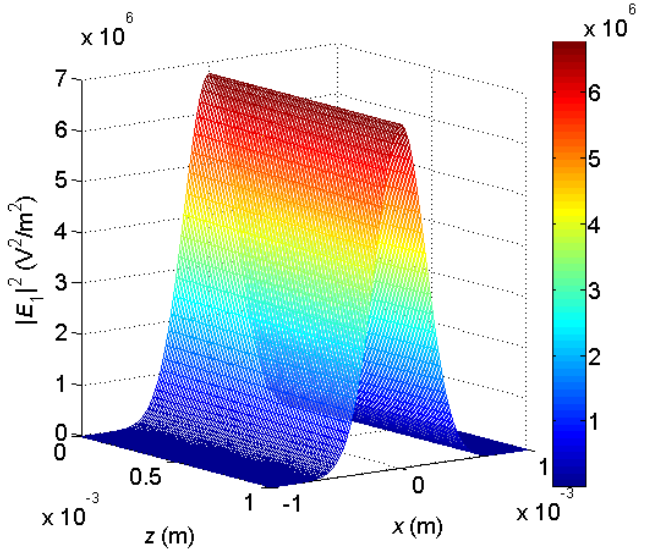

(a)

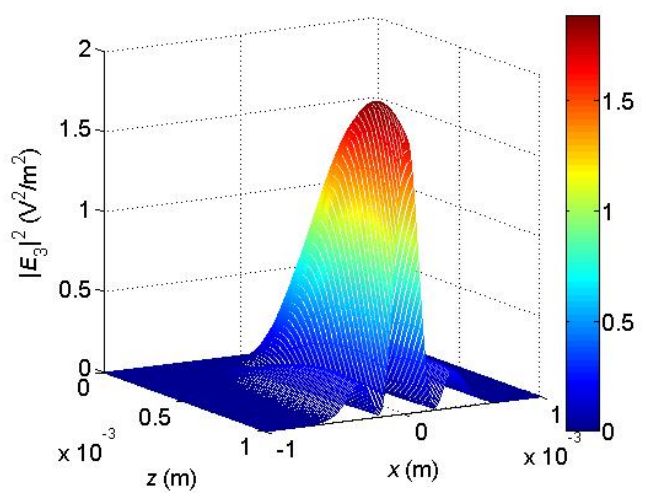

(c)

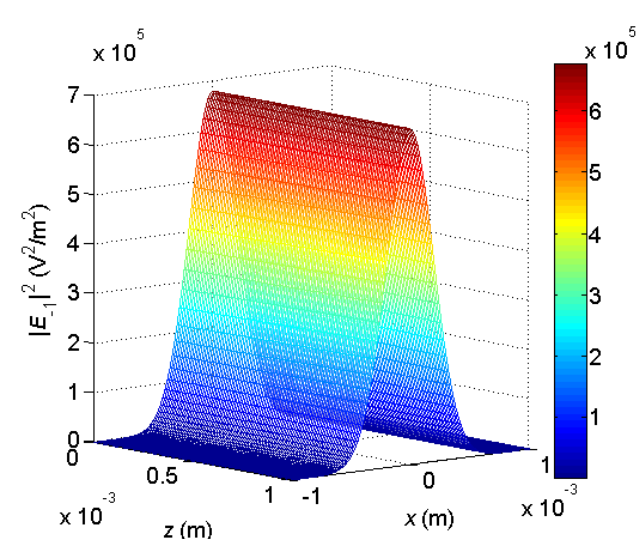

(b)

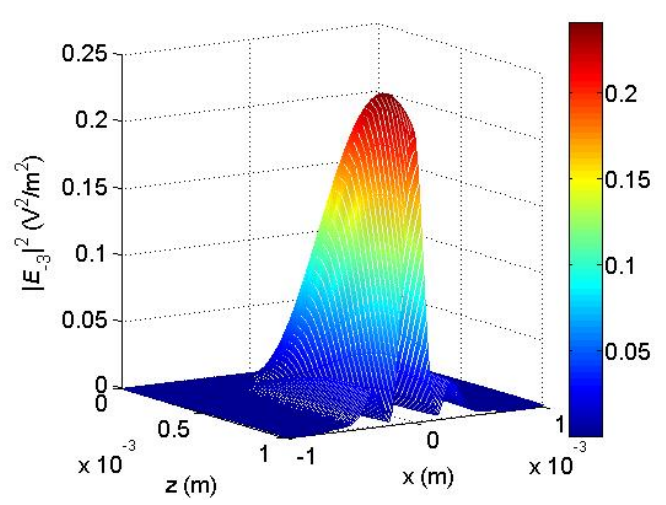

(d)

Figure 3.10: Spatial evolution of (a) first Bragg order $\left(E_{1}\right)$, (b) second Bragg order $\left(E_{-1}\right)$, (c) first non-Bragg order $\left(E_{3}\right)$, and (d) second non-Bragg order $\left(E_{-3}\right)$ in PR LN. $P_{1}=20 \mathrm{~mW}, W_{1}=0.4 \mathrm{~mm} ; P_{-1}=2 \mathrm{~mW}, W_{-1}=0.4 \mathrm{~mm}$.

\subsection{Bragg and Non-Bragg Orders for Incident Gaussian Beam Profiles with \\ Different Waists}

In this Section, Eqs. (2.9a-d) are numerically solved in MATLAB ${ }^{\odot}$ using Range-Kutta methods to demonstrate the spatial evolution of Bragg and non-Bragg orders in PR LN by varying the incident beam waists and keeping the incident beam powers equal. 
Numerical simulations are performed for the following two cases:

1) $W_{1}=1.6 \mathrm{~mm}, W_{-1}=0.4 \mathrm{~mm} ; P_{1}=P_{-1}=20 \mathrm{~mW} ; \theta=0.5^{\circ}$.

2) $W_{1}=2.4 \mathrm{~mm}, W_{-1}=0.3 \mathrm{~mm} ; P_{1}=P_{-1}=20 \mathrm{~mW} ; \theta=0.5^{\circ}$.

3.4.1 Numerical results for $W_{1}=1.6 \mathrm{~mm}, W_{-1}=0.4 \mathrm{~mm} ; P_{1}=P_{-1}=$ $20 \mathrm{~mW} ; \boldsymbol{\theta}=0.5^{\circ}$

Figures 3.11(a, b) show the incident Gaussian beam profiles of the pump (reference) and probe (object) beams.

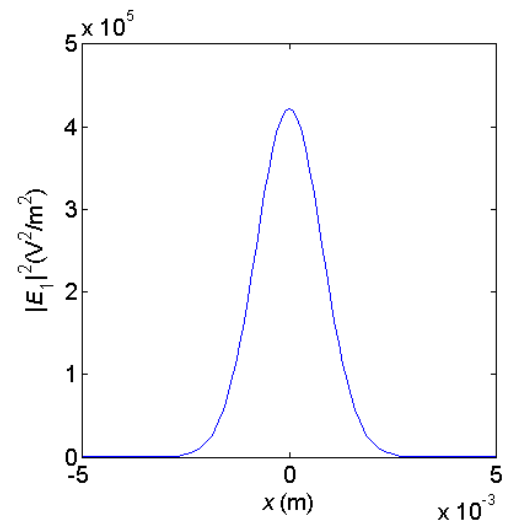

(a)

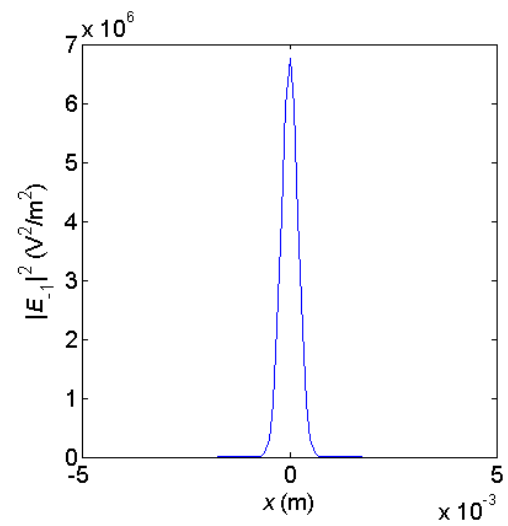

(b)

Figure 3.11: Gaussian beam profiles of incident (a) pump (reference) and (b) probe (object) beams. $P_{1}=20 \mathrm{~mW}, W_{1}=1.6 \mathrm{~mm} ; P_{-1}=20 \mathrm{~mW}, W_{-1}=0.4 \mathrm{~mm}$. The spatial shifts of $1 \mu \mathrm{m}$ is not discernible at this scale.

The pump (reference) and probe (object) are used as incident beams to solve Eqs. (2.9ad) in MATLAB ${ }^{\odot}$ to study the behavior of Bragg and non-Bragg orders inside PR LN. Figure 3.12(a)-(d) illustrate the spatial evolution of Bragg and non-Bragg orders in PR $\mathrm{LN}$ for the case where $W_{1}=1.6 \mathrm{~mm}, W_{-1}=0.4 \mathrm{~mm} ; P_{1}=P_{-1}=20 \mathrm{~mW} ; \theta=0.5^{\circ}$. 


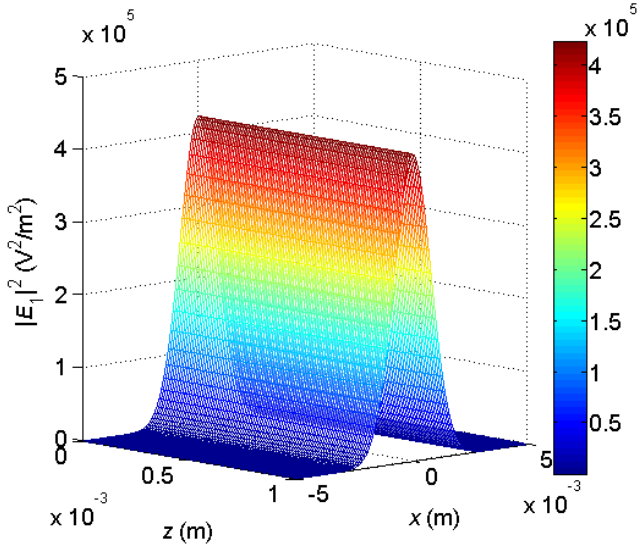

(a)

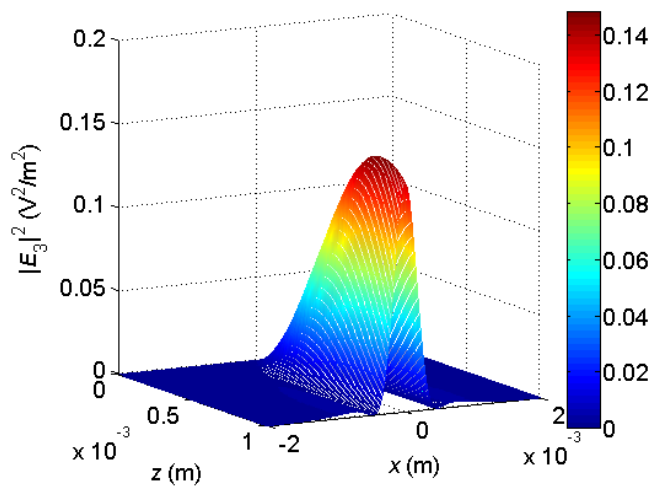

(c)

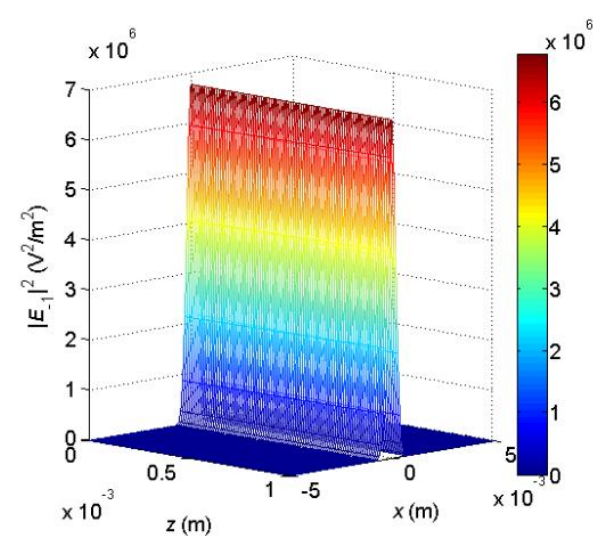

(b)

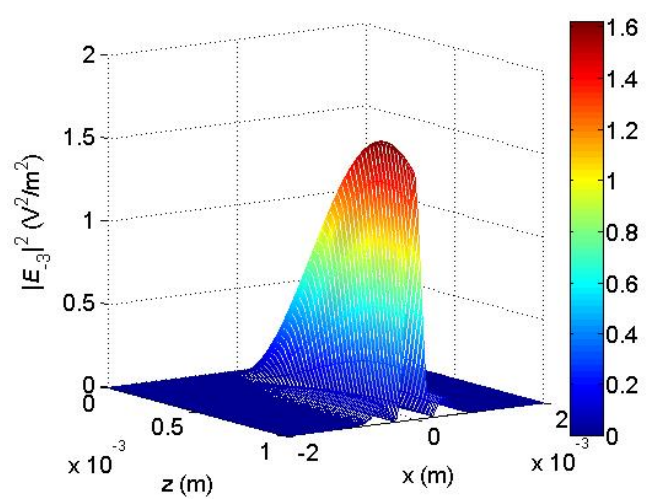

(d)

Figure 3.12: Spatial evolution of (a) first Bragg order $\left(E_{1}\right)$, (b) second Bragg order $\left(E_{-1}\right)$, (c) first non-Bragg order $\left(E_{3}\right)$, and (d) second non-Bragg order $\left(E_{-3}\right)$ in PR LN. $P_{1}=20 \mathrm{~mW}, W_{1}=1.6 \mathrm{~mm} ; P_{-1}=20 \mathrm{~mW}, W_{-1}=0.4 \mathrm{~mm}$.

Theoretically from Eqs. (2.6c) - (2.6d) the waists of higher orders can be calculated. From Eq. (2.6c) it is evident that the dominant term responsible for the evolution of first non-Bragg $\left(\mathrm{E}_{3}\right)$ order is $E_{1} E_{1} E_{-1}^{*}$. Here both $\mathrm{E}_{1}$ and $\mathrm{E}_{-1}$ have Gaussian beam profiles. Therefore the expression to calculate the width of the first non-Bragg order can be written as (assuming no mode conversion):

$$
E_{3} \approx A_{1} A_{1} A_{-1}^{*} e^{-x^{2}\left(\frac{1}{W_{1}^{2}}+\frac{1}{W_{1}^{2}}+\frac{1}{W_{-1}^{2}}\right)}
$$


In writing Eq. (3.3), the Gaussian form of the participating Bragg orders have been explicitly written. Similarly from Eq. (2.6d) it is evident that the dominant term responsible for the evolution of second non-Bragg $\left(\mathrm{E}_{-3}\right)$ order is $E_{-1} E_{-1} E_{1}^{*}$. Therefore the expression to calculate the width of the second non-Bragg order can be written as (assuming no mode conversion):

$$
E_{-3} \approx A_{-1} A_{-1} A_{1}^{*} e^{-x^{2}\left(\frac{1}{W_{-1}^{2}}+\frac{1}{W_{-1}^{2}}+\frac{1}{W_{1}^{2}}\right)}
$$

In this Section the waists $W_{1}, W_{-1}$ of the pump and probe beams are taken as $1.6 \mathrm{~mm}$ and $0.4 \mathrm{~mm}$, respectively. With these values being substituted in Eqs. (3.3) (3.4) the width of $\left|E_{3}\right|^{2}$ is $0.266 \mathrm{~mm}$ and that of $\left|E_{-3}\right|^{2}$ is $0.196 \mathrm{~mm}$, respectively. It is expected that the width of the intensity profile of the first non-Bragg order is greater than that of the second non-Bragg order, which is in agreement with the numerically obtained values of the widths of the main lobes of these orders, which are $0.247 \mathrm{~mm}$ and $0.154 \mathrm{~mm}$, respectively. Since in this example, $W_{1}=4 W_{-1}$, the ratio of the widths of the intensity profiles of the first non-Bragg order $(+3)$ to that of the second nonBragg order (-3) is approximately 1.36 , which is in close agreement with the ratio of 1.60 obtained from the simulations. As before, mode conversion is observed in the profiles of the non-Bragg orders.

\subsubsection{Numerical results for $W_{1}=2.4 \mathrm{~mm}, W_{-1}=0.3 \mathrm{~mm} ; P_{1}=P_{-1}=$ $20 \mathrm{~mW} ; \theta=0.5^{\circ}$}

Figures 3.13(a, b) show the incident Gaussian beam profiles of the pump (reference) and probe (object) beams. Figures 3.14(a)-(d) illustrate the spatial evolution of Bragg and non-Bragg orders in PR LN for the case where $W_{1}=2.4 \mathrm{~mm}, W_{-1}=$ $0.3 \mathrm{~mm} ; P_{1}=P_{-1}=20 \mathrm{~mW} ; \theta=0.5^{\circ}$. The widths of the non-Bragg orders are in agreement with the discussion in Section 3.4.1. 


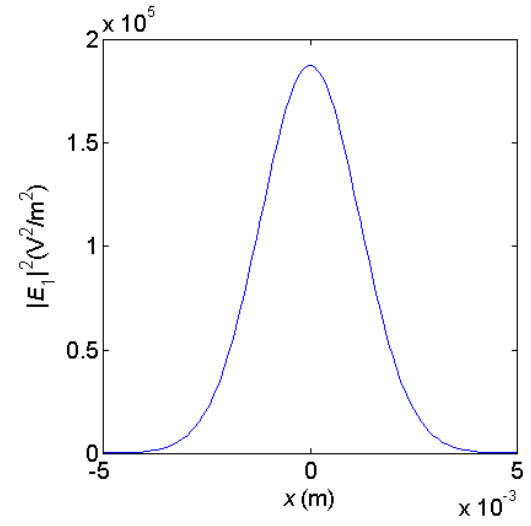

(a)

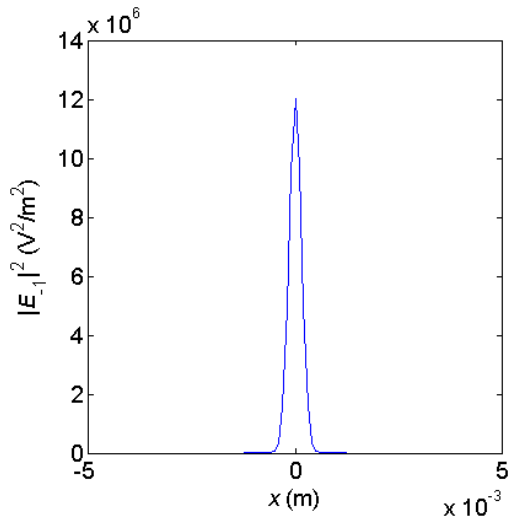

(b)

Figure 3.13: Gaussian beam profiles of incident (a) pump (reference) and (b) probe (object) beams. $P_{1}=20 \mathrm{~mW}, W_{1}=2.4 \mathrm{~mm} ; P_{-1}=20 \mathrm{~mW}, W_{-1}=0.3 \mathrm{~mm}$. The spatial shifts of $1 \mu \mathrm{m}$ is not discernible at this scale.

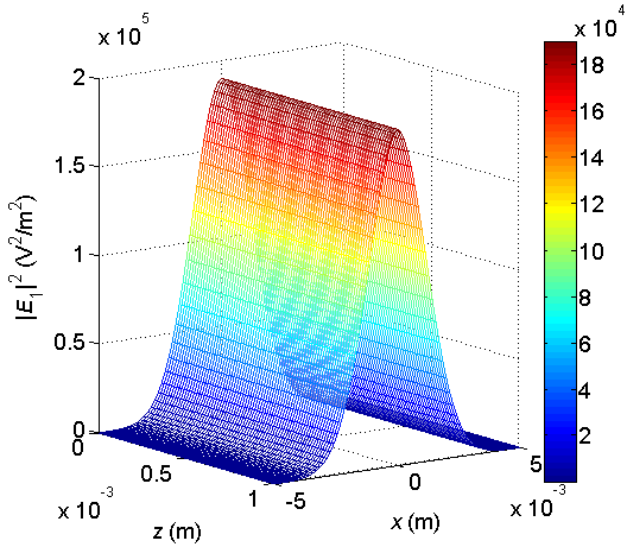

(a)

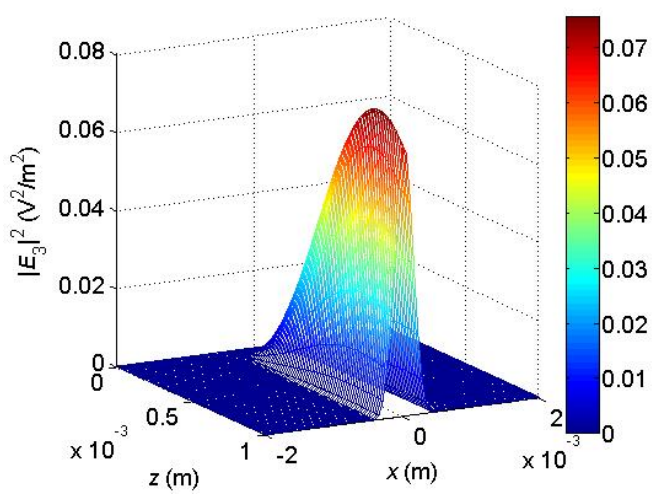

(c)

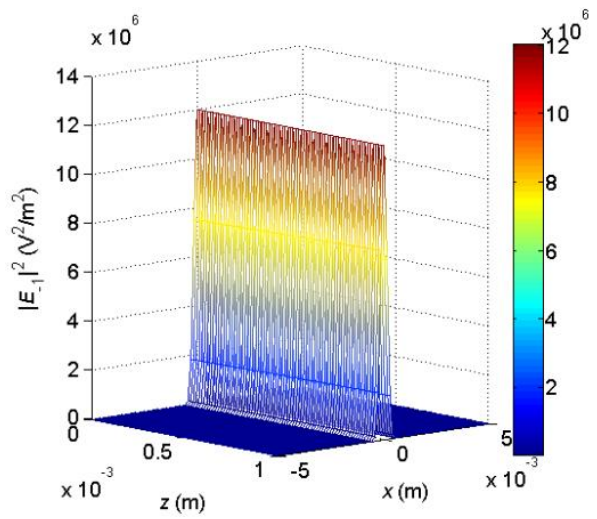

(b)

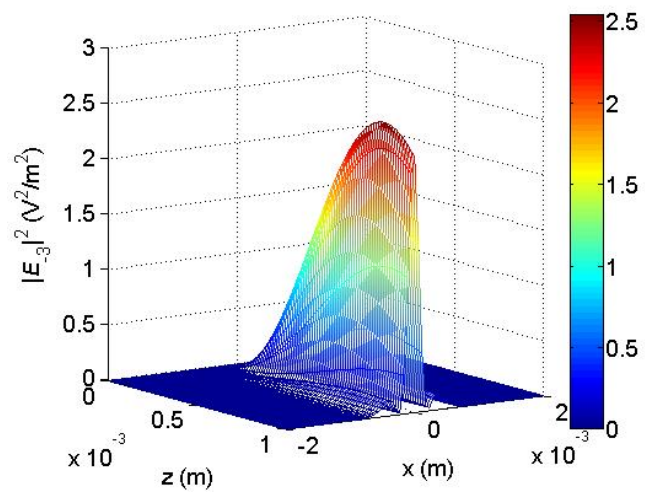

(d)

Figure 3.14: Spatial evolution of (a) first Bragg order $\left(E_{1}\right)$, (b) second Bragg order $\left(E_{-1}\right)$, (c) first non-Bragg order $\left(E_{3}\right)$, and (d) second non-Bragg order $\left(E_{-3}\right)$ in PR LN. $P_{1}=20 \mathrm{~mW}, W_{1}=2.4 \mathrm{~mm} ; P_{-1}=20 \mathrm{~mW}, W_{-1}=0.3 \mathrm{~mm}$. 
From Figure 3.14(a)-(d) it is evident that higher or non-Bragg orders are generated at the expense of energy from +1 and -1 (Bragg) orders.

Interestingly from Figure 3.14(c) the profile of first non-Bragg order at the exit plane of crystal is approximately Gaussian, and no side lobes are observed. From this it can be concluded that by reducing the waist of probe beam by at least 8 times compared to that of pump beam, the effect of mode conversion of incident beam profile in first non-Bragg order can be avoided. But it is important to note that still the effect of mode conversion is observed during the evolution of second non-Bragg order. Also another key observation is the process of mode conversion cannot be avoided completely in both the higher orders by making both the pump and probe beams wider.

By varying the waists of incident beam profiles the higher orders are numerically analyzed and also a way to suppress the process of the mode conversion in one of the higher orders has been identified. In the next Section a through numerical analysis is performed by varying the angle between the incident beams.

\subsection{Bragg and Non-Bragg Orders for Incident Gaussian Beam Profiles with Different Interaction Angles: Intensity and Phase}

In this Section, the effect on higher order generation has been investigated by varying the angle between the incident beam profiles. Numerical simulations are performed for four different cases:

1) $P_{1}=P_{-1}=20 \mathrm{~mW} ; W_{1}=W_{-1}=2.4 \mathrm{~mm} ; \theta=0.5^{\circ}$.

2) $P_{1}=P_{-1}=20 \mathrm{~mW} ; W_{1}=W_{-1}=2.4 \mathrm{~mm} ; \theta=1^{\circ}$.

3) $P_{1}=P_{-1}=20 \mathrm{~mW} ; W_{1}=W_{-1}=2.4 \mathrm{~mm} ; \theta=2^{\circ}$.

4) $P_{1}=P_{-1}=20 \mathrm{~mW} ; W_{1}=W_{-1}=0.6 \mathrm{~mm} ; \theta=2^{\circ}$. 
When the external angles are $0.5^{\circ}, 1^{\circ}$ and $2^{\circ}$, then the corresponding values of $|a|$ are $1 \mu \mathrm{m}, 2 \mu \mathrm{m}$ and $4 \mu \mathrm{m}$, respectively. Furthermore, the phases of the Bragg and nonBragg orders are investigated, with potential applications to PSDH in mind.

3.5.1 Numerical results for $P_{1}=P_{-1}=20 \mathrm{~mW} ; W_{1}=W_{-1}=2.4 \mathrm{~mm} ; \theta=0.5^{\circ}$

Figures 3.15(a)-(d) illustrate the spatial evolution of Bragg and non-Bragg orders in PR LN for the case where $P_{1}=P_{-1}=20 \mathrm{~mW} ; W_{1}=W_{-1}=2.4 \mathrm{~mm} ; \theta=$ $0.5^{\circ}$

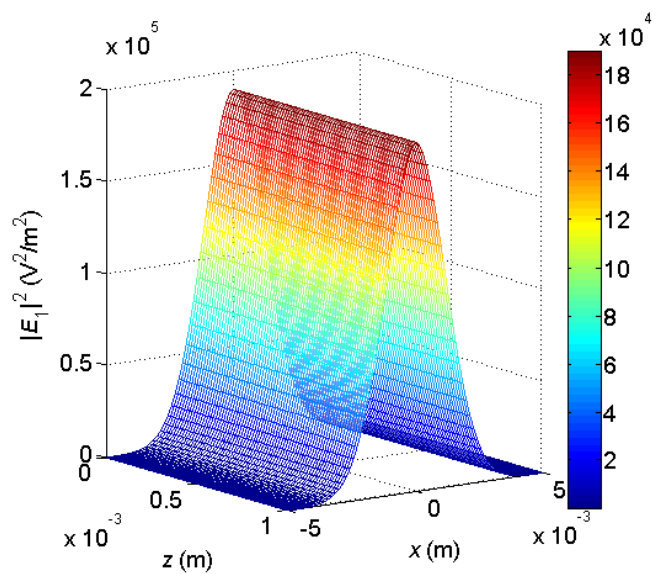

(a)

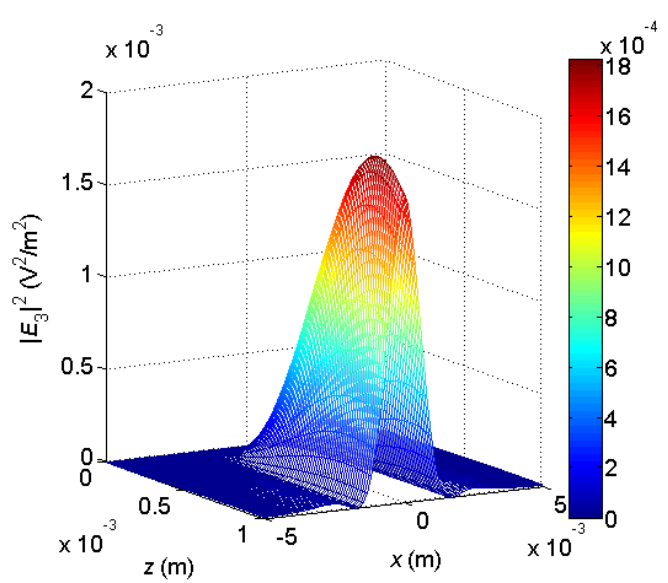

(c)

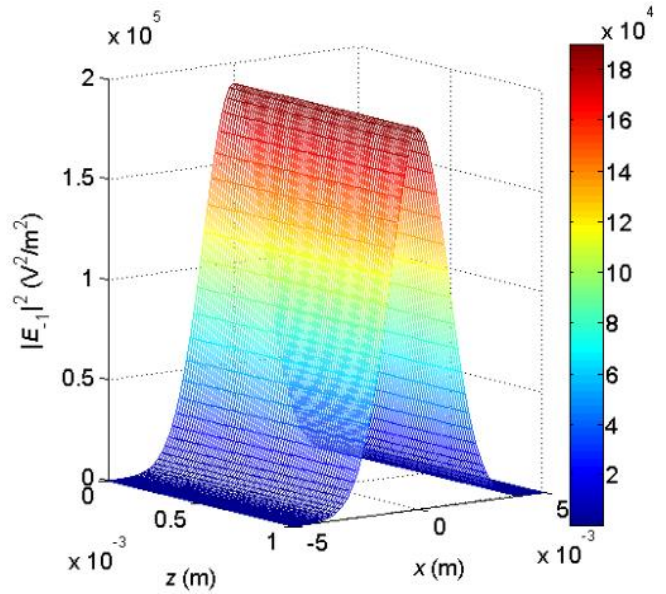

(b)

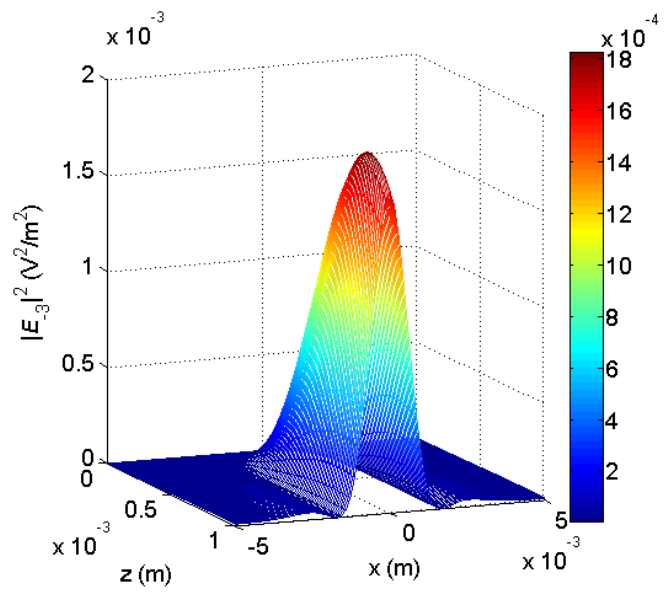

(d)

Figure 3.15: Spatial evolution of (a) first Bragg order $\left(E_{1}\right)$, (b) second Bragg order $\left(E_{-1}\right)$, (c) first non-Bragg order $\left(E_{3}\right)$, and (d) second non-Bragg order $\left(E_{-3}\right)$ in PR LN. $P_{1}=20 \mathrm{~mW}, W_{1}=2.4 \mathrm{~mm} ; P_{-1}=20 \mathrm{~mW}, W_{-1}=2.4 \mathrm{~mm} ; \theta=0.5^{\circ}$. 
Comparison with Figures 3.3(a)-(d) show decreased mode conversion, possibly due to the increased waist of the interacting Gaussian beams. When the incident beams have wider Gaussian profiles the effect of mode conversion in higher orders can be reduced but it cannot be suppressed completely. The on-axis variations of the Bragg and non-Bragg orders are qualitatively similar to that in Figure 3.4(a)-(d).

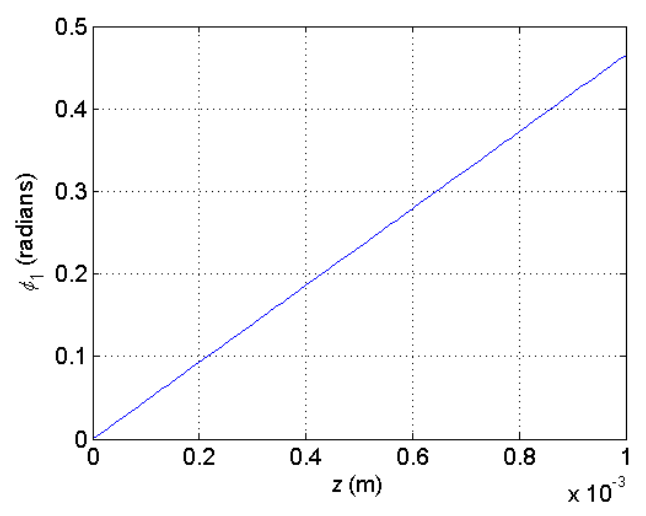

(a)

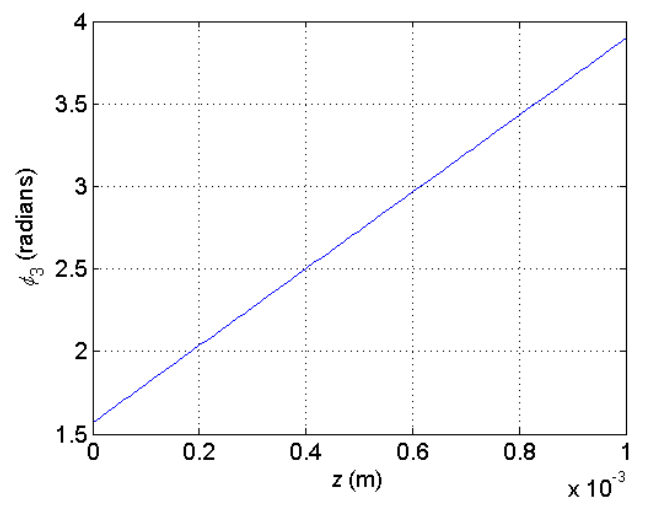

(c)

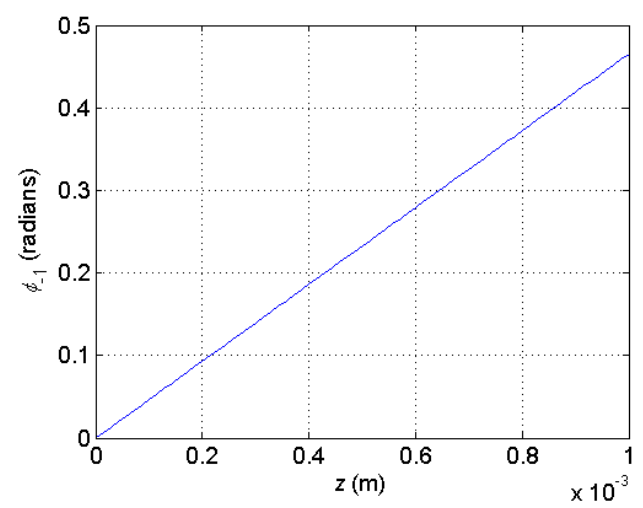

(b)

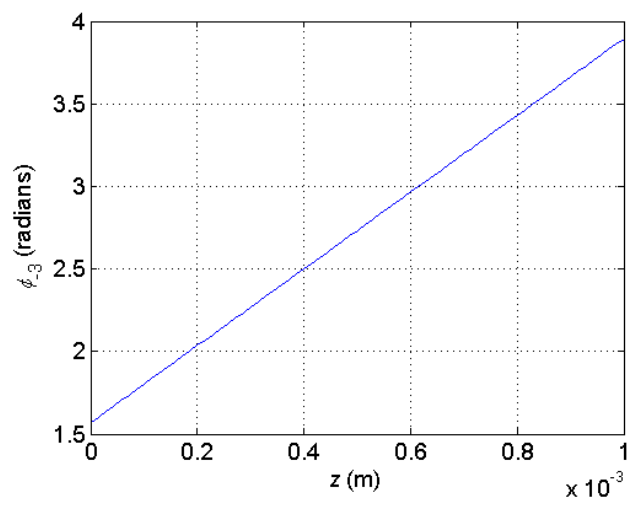

(d)

Figure 3.16: Unwrapped on-axis $(x=0)$ phase profile of (a) first Bragg order $\left(E_{1}\right)$, (b) second Bragg order $\left(E_{-1}\right)$, (c) first non-Bragg order $\left(E_{3}\right)$, and (d) second non-Bragg $\operatorname{order}\left(E_{-3}\right)$ during propagation.

Figures 3.16(a)-(d) illustrate the unwrapped on-axis phase profiles of Bragg and non-Bragg orders when the angle between the incident beams is $0.5^{\circ}$. While the phases of the Bragg orders starts from 0 and increase linearly, the phases of the non-Bragg orders start from $\pi / 2$ and linearly increase. It is noted that the phases of the first set of 
higher (non-Bragg) orders during Raman-Nath diffraction are different from the phases of the Bragg orders by $\pi / 2$.

3.5.2 Numerical results for $P_{1}=P_{-1}=20 \mathrm{~mW} ; W_{1}=W_{-1}=2.4 \mathrm{~mm} ; \theta=1^{\circ}$

Figures 3.17(a)-(d) illustrate the spatial evolution of Bragg and non-Bragg orders in PR LN for the case where $P_{1}=P_{-1}=20 \mathrm{~mW} ; W_{1}=W_{-1}=2.4 \mathrm{~mm} ; \theta=$ $1^{\circ}$. Figures 3.18 (a), (b) show the on-axis intensity variation of non-Bragg orders in PR LN. 


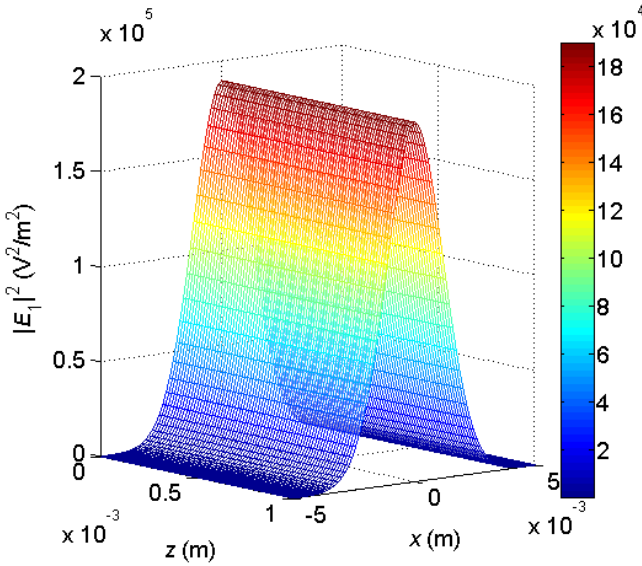

(a)

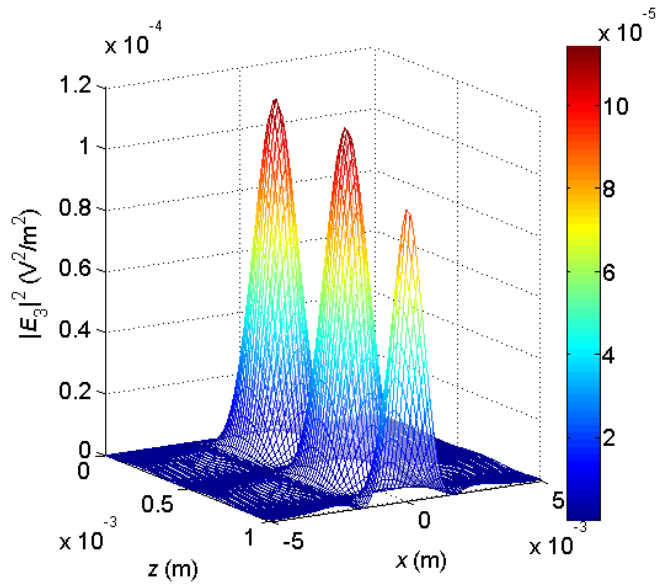

(c)

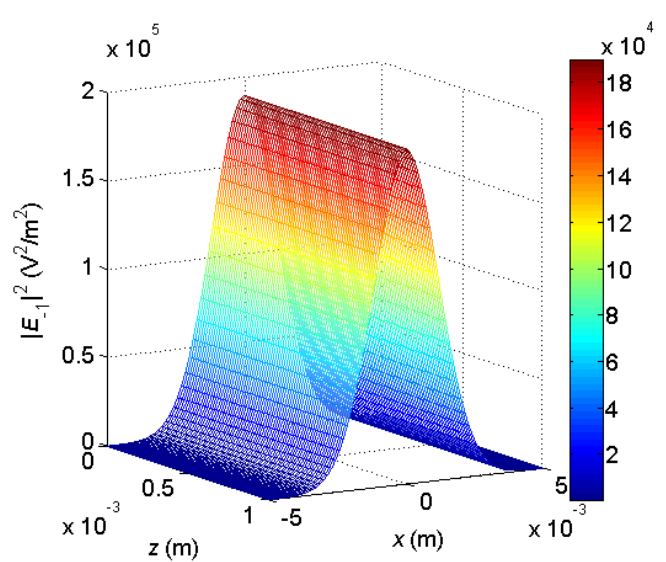

(b)

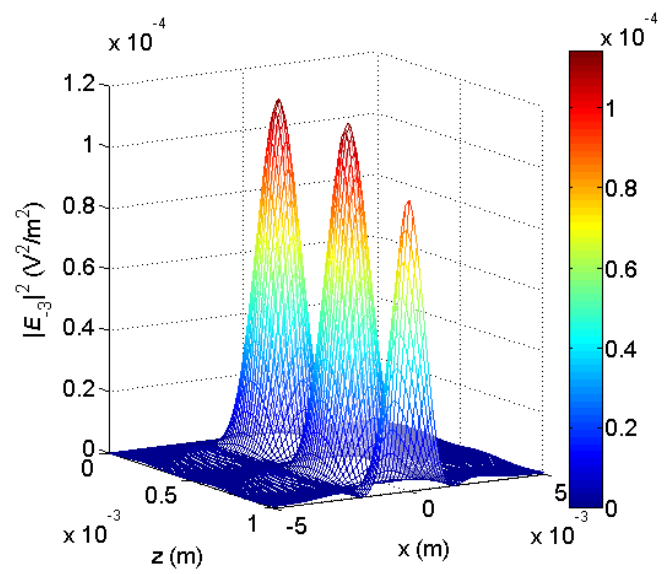

(d)

Figure 3.17: Spatial evolution of (a) first Bragg order $\left(E_{1}\right)$, (b) second Bragg order $\left(E_{-1}\right)$, (c) first non-Bragg order $\left(E_{3}\right)$, and (d) second non-Bragg order $\left(E_{-3}\right)$ in PR LN. $P_{1}=20 \mathrm{~mW}, W_{1}=2.4 \mathrm{~mm} ; P_{-1}=20 \mathrm{~mW}, W_{-1}=2.4 \mathrm{~mm} ; \theta=1^{\circ}$. 


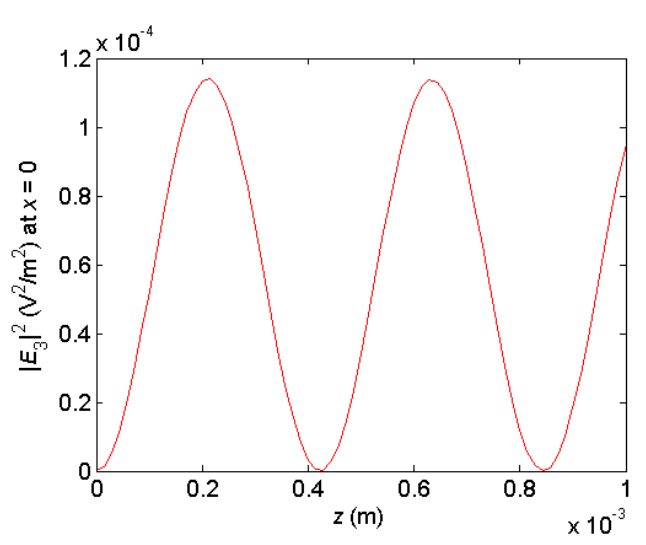

(a)

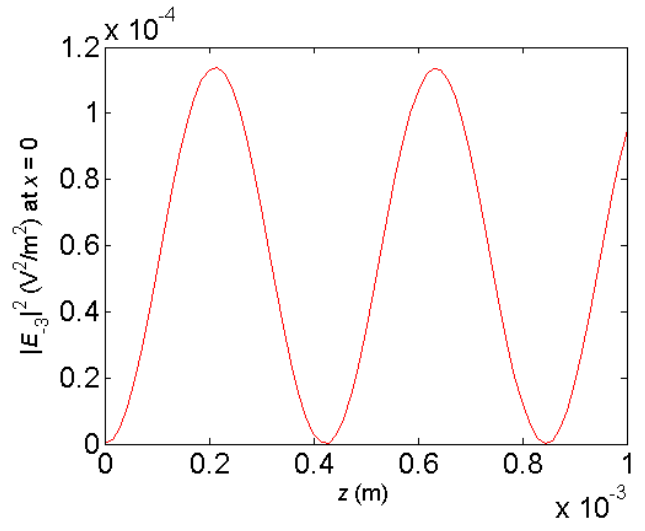

(b)

Figure 3.18: Variation of on-axis $(x=0)$ intensity of (a) first non-Bragg order $\left(E_{3}\right)$, and (b) second non-Bragg order $\left(E_{-3}\right)$ during propagation.

As before, higher or non-Bragg orders are once again generated at the expense of energy from +1 and -1 (Bragg) orders. Furthermore, the intensities of the first and second non-Bragg orders are identical, since the incident beams have identical powers; however, as is evident from comparing Figures 3.15 and 3.17, their peak intensities are less than for the case of smaller incident angles of the Gaussian beams. Also there is a quasi-periodic (and identical) axial variation of the higher order intensities as they propagate through the crystal, with the period being approximately $0.864 \mathrm{~mm}$, as determined from Figure 3.18. It is surmised that this period decreases with increasing interaction angle, by examining Figures 3.15 and 3.17. As shown in Figure 3.19, the phases of the non-Bragg orders once again start from $\pi / 2$; however, in this case, they also show a quasi-periodic variation with the same period as that of the intensity. On the other hand, the phases of the Bragg orders linearly increase from zero; however, they increase much more than for the case when the interaction angle is smaller (see Figures 3.16 and 3.19). 


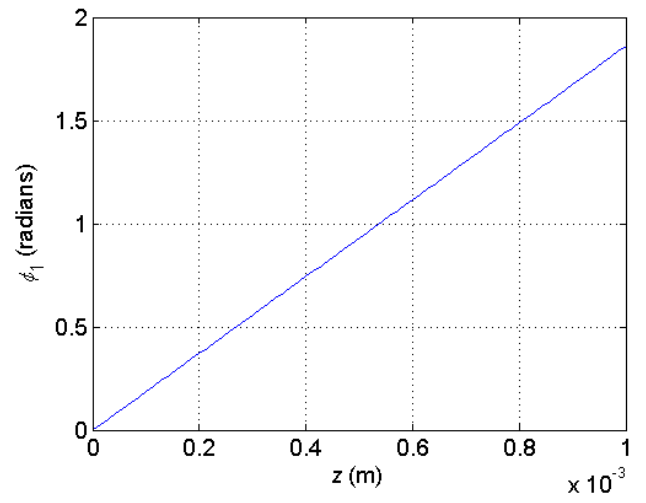

(a)

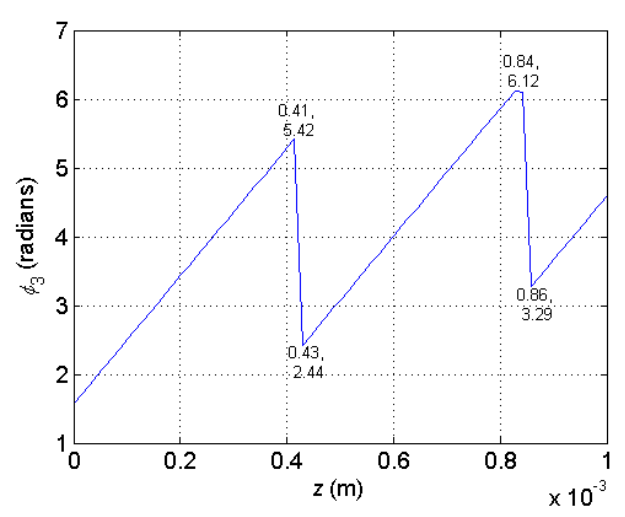

(c)

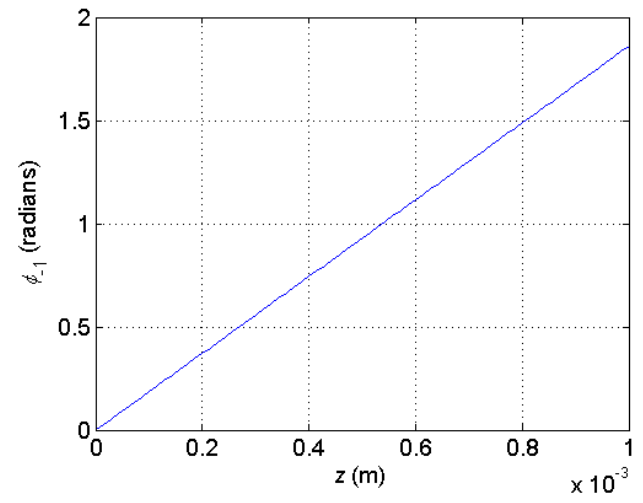

(b)

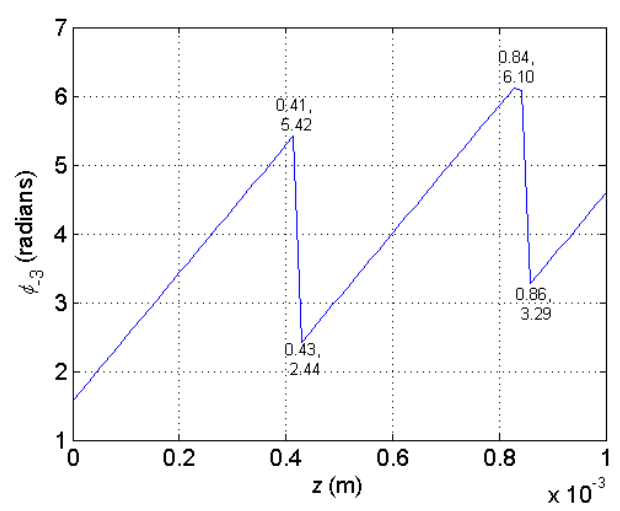

(d)

Figure 3.19: Unwrapped on-axis ( $x=0$ ) phase profile of (a) first Bragg order $\left(E_{1}\right)$, (b) second Bragg order $\left(E_{-1}\right)$, (c) first non-Bragg order $\left(E_{3}\right)$, and (d) second non-Bragg order $\left(E_{-3}\right)$ during propagation.

3.5.3 Numerical results for $P_{1}=P_{-1}=20 \mathrm{~mW} ; W_{1}=W_{-1}=2.4 \mathrm{~mm} ; \theta=2^{\circ}$

The plots for the evolution of the intensity profiles of the Bragg and non-Bragg orders are shown in Figure 3.20. On-axis intensity and phase variations are shown in Figures 3.21 and 3.22, respectively. The simulations confirm our earlier postulates that the peak intensity of the non-Bragg orders and spatial period of the variation of their on-axis intensities and phases decreases with increasing interaction angle. The phase variation of the Bragg orders are larger with larger interaction angle. Also, mode conversion is less evident with increase in the interaction angle. 


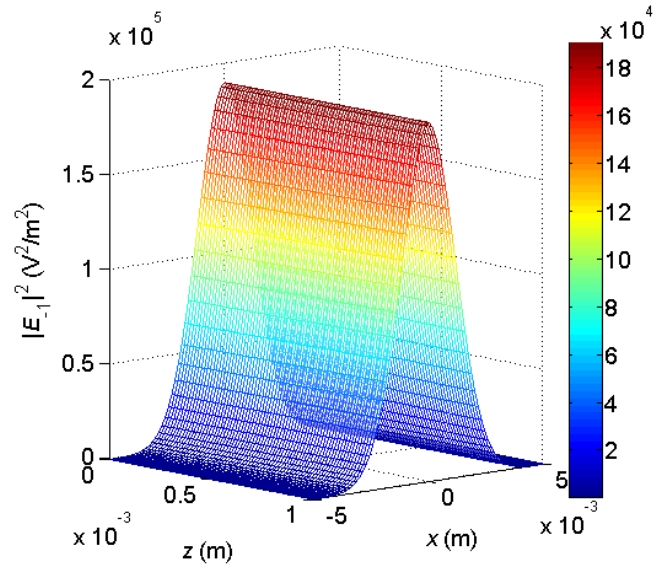

(a)

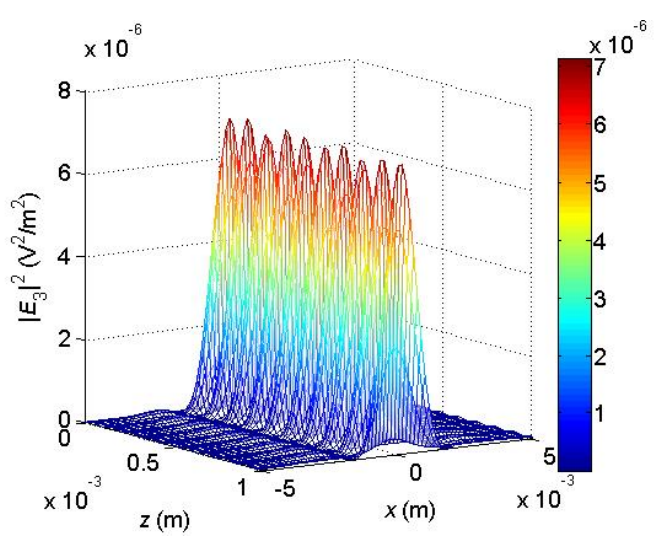

(c)

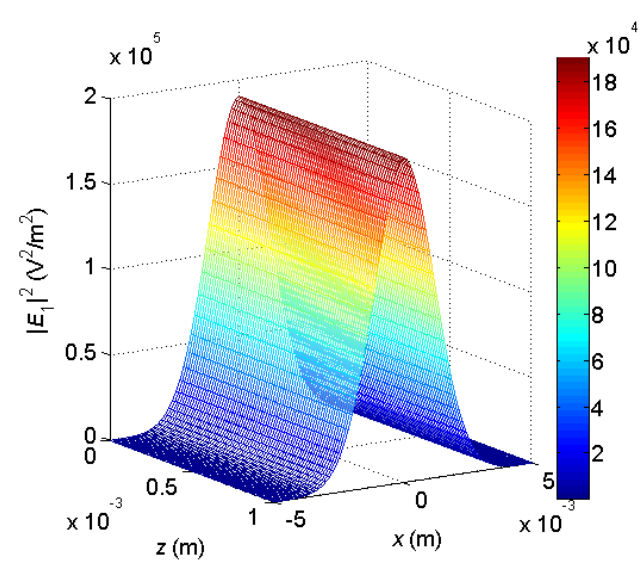

(b)

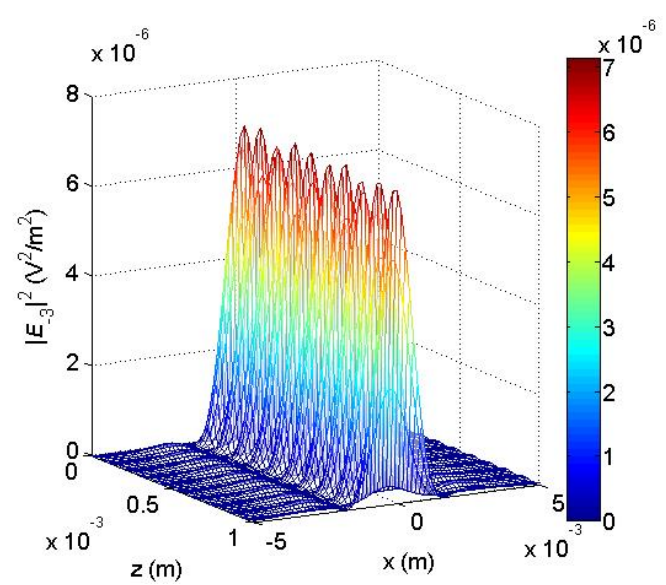

(d)

Figure 3.20: Spatial evolution of (a) first Bragg order $\left(E_{1}\right)$, (b) second Bragg order $\left(E_{-1}\right)$, (c) first non-Bragg order $\left(E_{3}\right)$, and (d) second non-Bragg order $\left(E_{-3}\right)$ in PR LN. $P_{1}=20 \mathrm{~mW}, W_{1}=2.4 \mathrm{~mm} ; P_{-1}=20 \mathrm{~mW}, W_{-1}=2.4 \mathrm{~mm} ; \theta=2^{\circ}$.

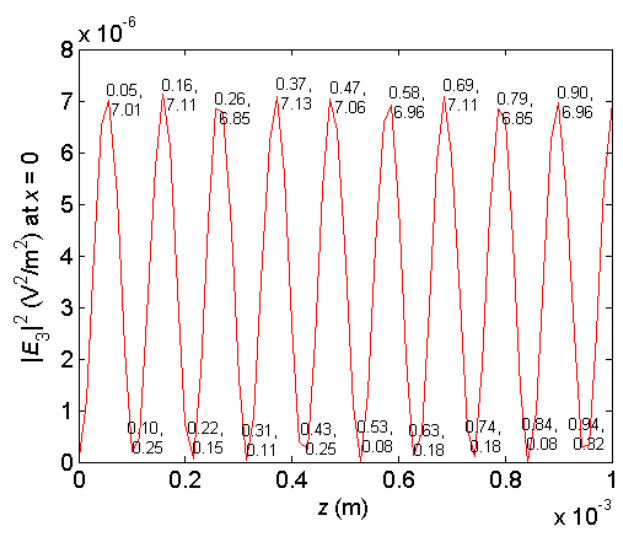

(a)

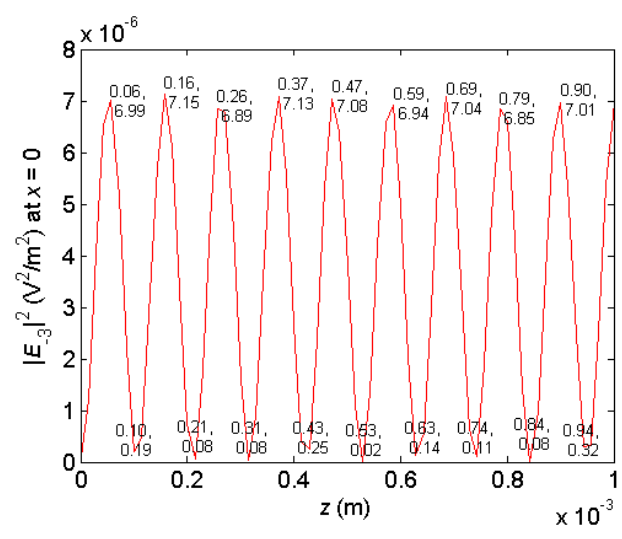

(b)

Figure 3.21: Variation of on-axis $(x=0)$ intensity of (a) first non-Bragg order $\left(E_{3}\right)$, and (b) second non-Bragg order $\left(E_{-3}\right)$ during propagation. 


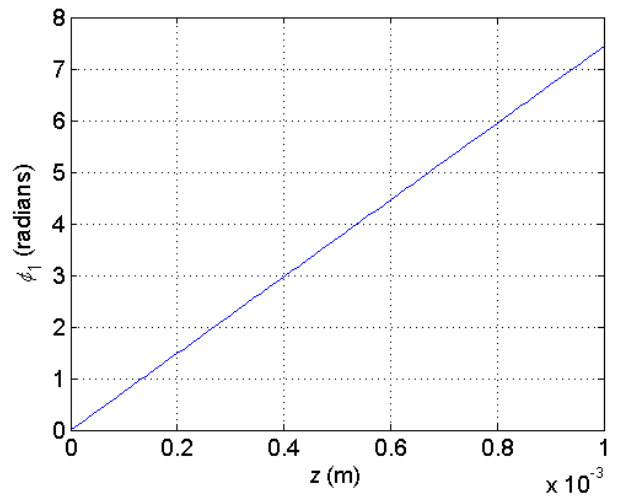

(a)

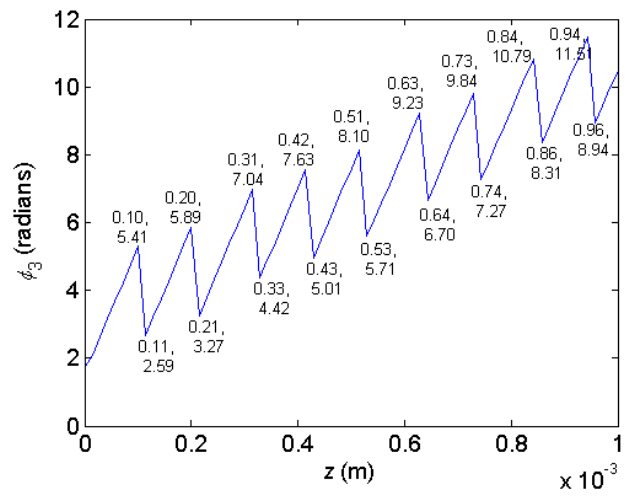

(c)

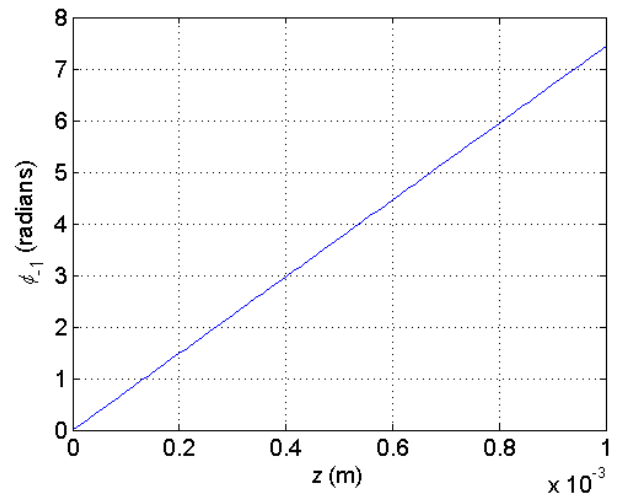

(b)

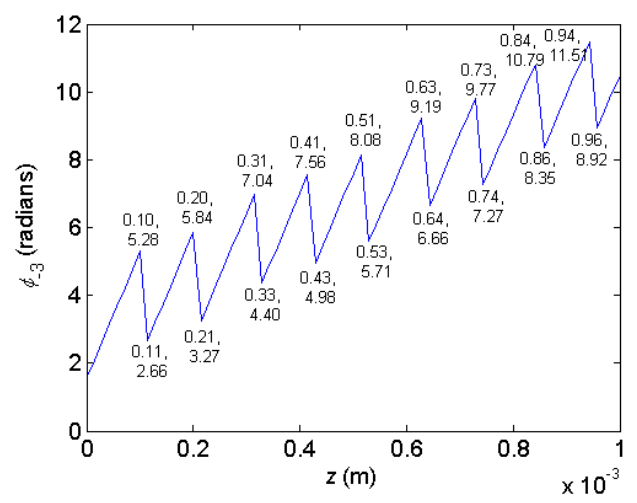

(d)

Figure 3.22: Unwrapped on-axis ( $x=0$ ) phase profile of (a) first Bragg order $\left(E_{1}\right)$, (b) second Bragg order $\left(E_{-1}\right)$, (c) first non-Bragg order $\left(E_{3}\right)$, and (d) second non-Bragg order $\left(E_{-3}\right)$ during propagation.

Finally, the dependence of the spatial evolution of the intensity profiles, on-axis intensities and phases and mode conversion on the waists of the interacting incident Gaussian is studied through the example below.

3.5.4 Numerical results for $P_{1}=P_{-1}=20 \mathrm{~mW} ; W_{1}=W_{-1}=0.6 \mathrm{~mm} ; \theta=2^{\circ}$

With the waist of the interacting Gaussians decreased to a quarter of the value in the preceding examples, the variations of the intensity profiles, on-axis intensities and phases are shown in Figures 3.22, 3.23, and 3.24, respectively. More pronounced mode conversion, presumably to higher-order Gaussians is evident from Figure 3.22. The peak intensities of the non-Bragg orders are higher compared to the previous 
example due to the fact that the peak intensities of the incident Gaussians is higher as well. The repetition period is approximately unchanged; however, the maxima of the on-axis intensities of the non-Bragg orders decrease with propagation distance in the PR material. This may be attributable to the fact that the interaction volume is less due to the decreased beam waist of the incident beams. The variation of the phases of the various orders are similar to that in the previous example and seem to be relatively unaffected by the decrease in the waist size of the incident beams.

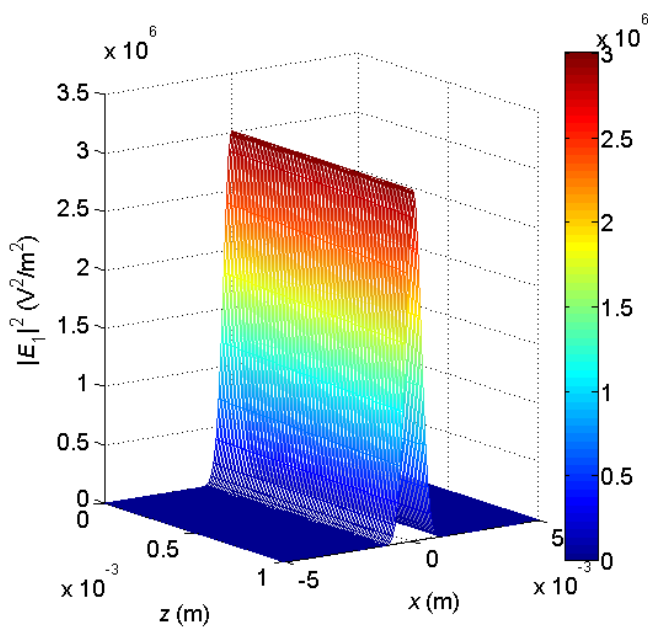

(a)

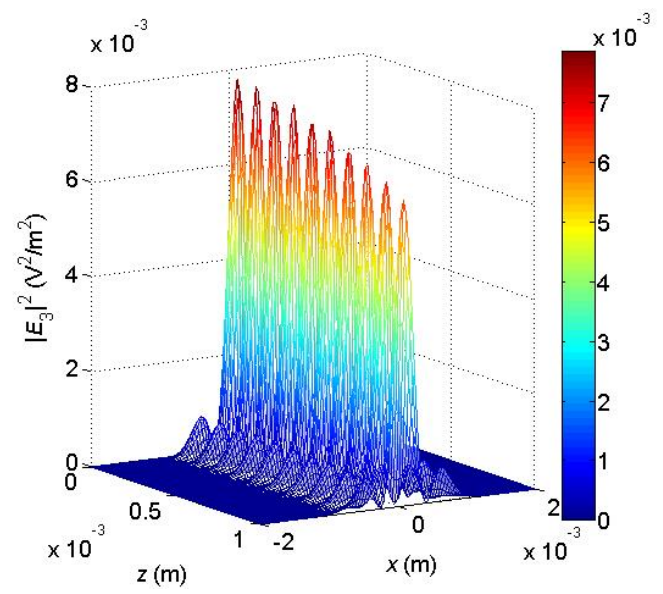

(c)

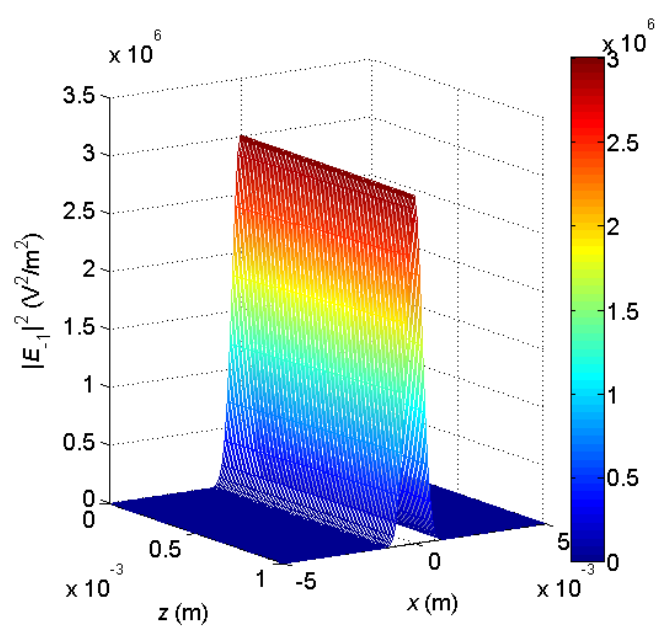

(b)

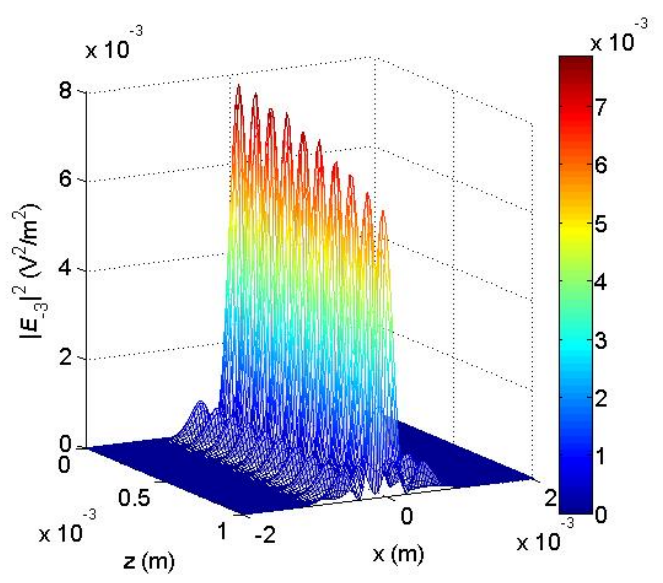

(d)

Figure 3.23: Spatial evolution of (a) first Bragg order $\left(E_{1}\right)$, (b) second Bragg order $\left(E_{-1}\right)$ (c) first non-Bragg order $\left(E_{3}\right)$, and (d) second non-Bragg order $\left(E_{-3}\right)$ in PR LN. $P_{1}=20 \mathrm{~mW}, W_{1}=0.6 \mathrm{~mm} ; P_{-1}=20 \mathrm{~mW}, W_{-1}=0.6 \mathrm{~mm} ; \theta=2^{\circ}$. 


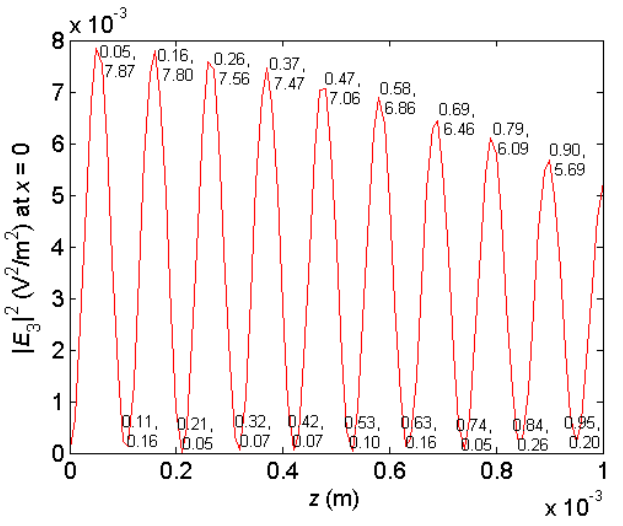

(a)

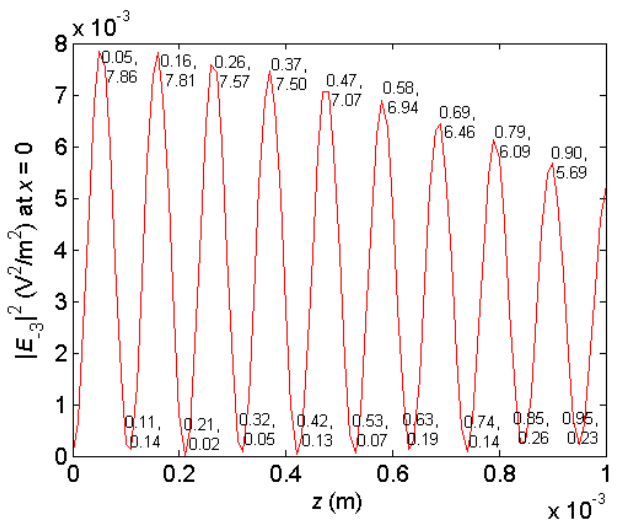

(b)

Figure 3.24: Variation of on-axis $(x=0)$ intensity of (a) first non-Bragg order $\left(E_{3}\right)$, and (b) second non-Bragg order $\left(E_{-3}\right)$ during propagation.

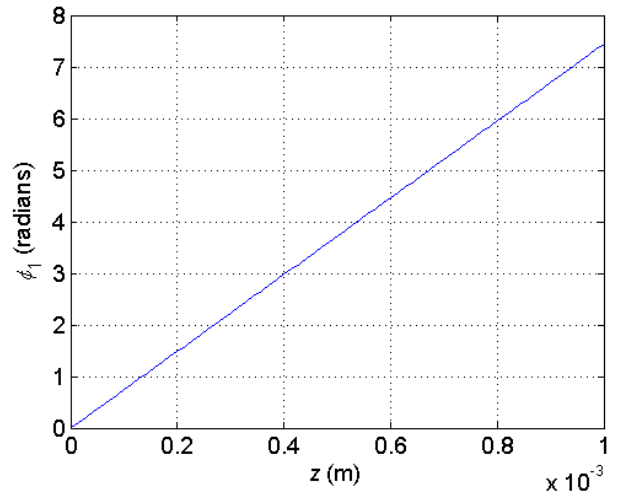

(a)

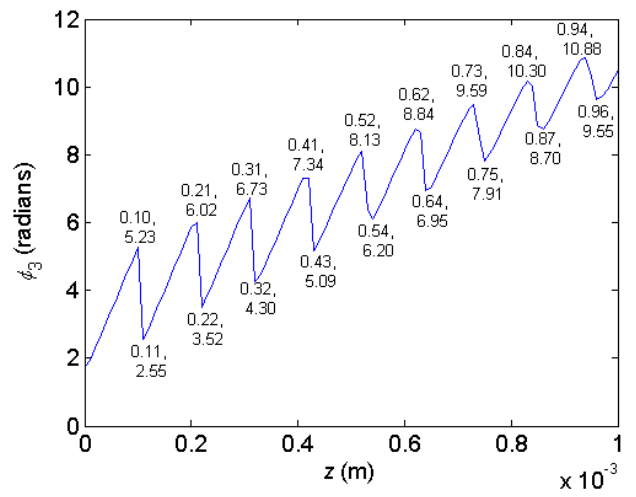

(c)

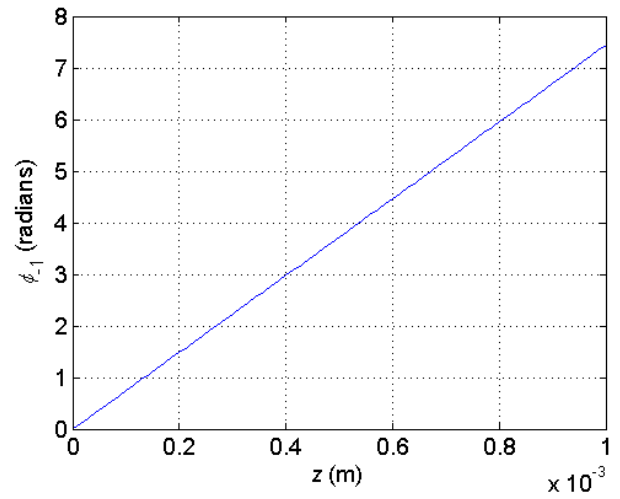

(b)

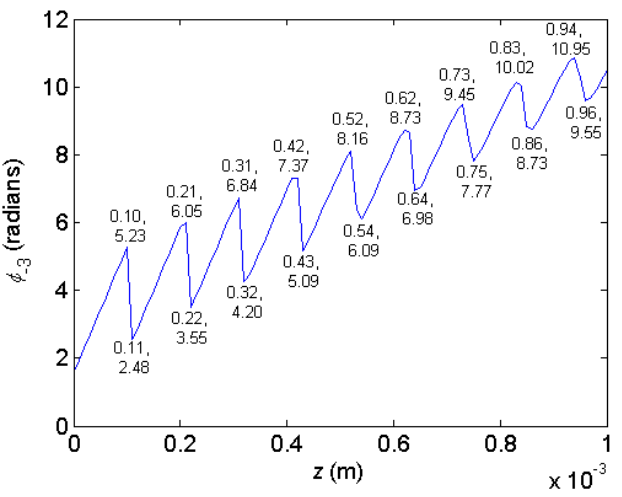

(d)

Figure 3.25: Unwrapped on-axis $(x=0)$ phase profile of (a) first Bragg order $\left(E_{1}\right),(\mathrm{b})$ second Bragg order $\left(E_{-1}\right)$ (c) first non-Bragg order $\left(E_{3}\right)$, and (d) second non-Bragg order $\left(E_{-3}\right)$ during propagation. 


\subsection{Conclusion}

In this Chapter the exact solutions to the interaction equations of Bragg and nonBragg orders are obtained by solving them numerically in MATLAB $^{\odot}$ for a variety of Gaussian beam profiles. A thorough numerical analysis is performed to study the behavior of non-Bragg orders during their evolution inside PR material by varying all the incident beam parameters such as beam powers, beam waists and also the angle between them. It can be concluded that by varying the incident beam waists, the effect of mode conversion in higher non-Bragg orders can be controlled in a significant manner. Mode conversion may be due to the fact that the higher non-Bragg order beams after being generated travel at an angle and therefore encounter an induced refractive index profile which appear more asymmetric than what is seen by the Bragg orders. Also, with increasing angle between the incident beams, the "launch" angle for the nonBragg orders also increases, resulting in a smaller value for the repetition period. Also by varying the angle between incident beams the amplitudes or powers of higher orders can be either increased or decreased.

In the following Chapter a similar analysis is performed, but the incident beam profiles used are super-Gaussians (flattops) instead of Gaussians, along with a superGaussian for one incident beam profile and a Gaussian beam profile for the other. 


\section{CHAPTER IV}

\section{SPECTRAL ANALYSIS OF BRAGG AND NON-BRAGG ORDERS USING ARBITRARY BEAM PROFILES}

\subsection{Introduction}

From Chapter 3, it is evident that the effect of mode conversion in the higher orders depends on the waists of incident beam profiles. Also the spatial periodic variation of the higher non-Bragg orders depends on the external angle between the incident beams.

In this Chapter, the interaction of other beam profiles such as two superGaussians (or flat-tops) and between a super-Gaussian and a Gaussian are investigated, with particular attention to mode conversion, spatial periodicity during interaction specially for the non-Bragg orders as well as the evolution of the phases of the Bragg and non-Bragg orders.

\subsection{Bragg and Non-Bragg Orders for Different Incident Flattop Beam Profiles}

In this Section, the effect of mode conversion in the generation of higher orders has been investigated for two flattop beam profiles by varying their waists and the angle between them. Numerical simulations are shown for two illustrative cases:

1) $P_{1}=P_{-1}=20 \mathrm{~mW} ; W_{1}=3.6 \mathrm{~mm}, W_{-1}=0.6 \mathrm{~mm} ; \theta=0.5^{\circ}$.

2) $P_{1}=P_{-1}=20 \mathrm{~mW} ; W_{1}=W_{-1}=2.4 \mathrm{~mm} ; \theta=2^{\circ}$. 
4.2.1 Numerical results for $P_{1}=P_{-1}=20 \mathrm{~mW} ; W_{1}=3.6 \mathrm{~mm}, W_{-1}=$ $0.6 \mathrm{~mm} ; \theta=0.5^{\circ}$.

The incident flat-top beam profiles are shown in Figure 4.1, and the evolution of the profiles of Bragg and non-Bragg orders are shown in Figure 4.2. The flat-tops are generated using a super-Gaussian function $\exp -\left(\frac{x^{2}}{W^{2}}\right)^{n}$ with $n=8$.

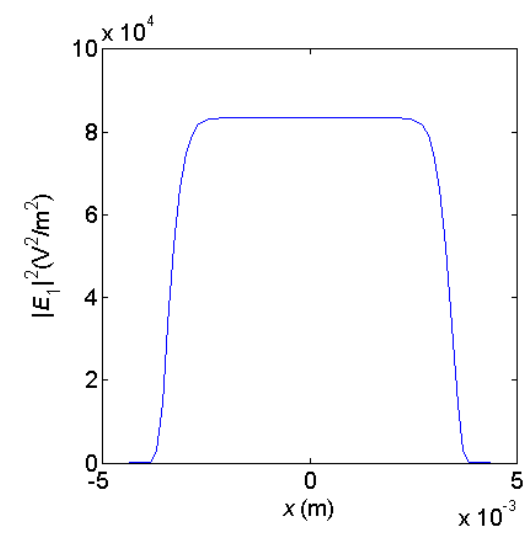

(a)

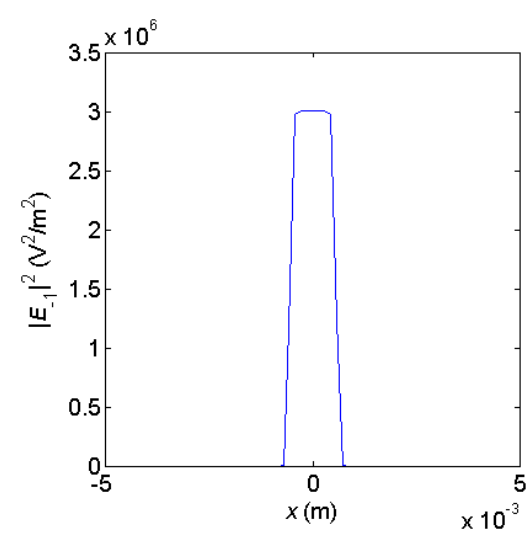

(b)

Figure 4.1: Flattop beam profiles of incident (a) pump (reference) and (b) probe (object) beams. $P_{1}=20 \mathrm{~mW}, W_{1}=3.6 \mathrm{~mm} ; P_{-1}=20 \mathrm{~mW}, W_{-1}=0.6 \mathrm{~mm}$. 


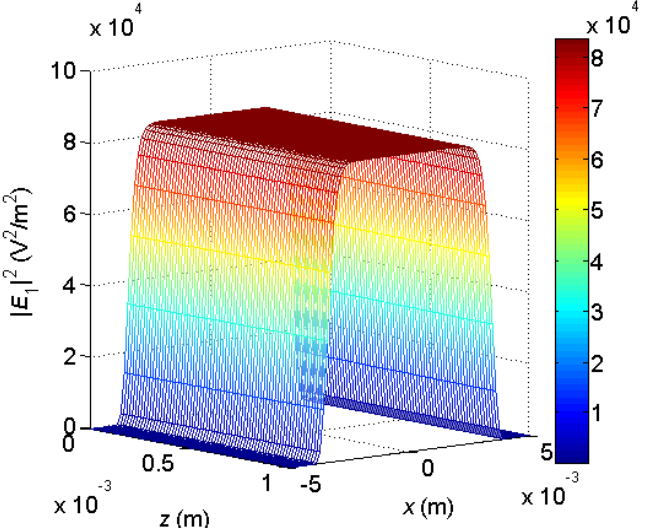

(a)

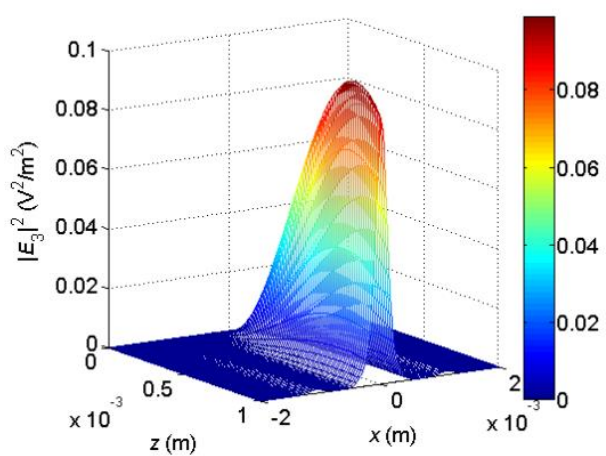

(c)

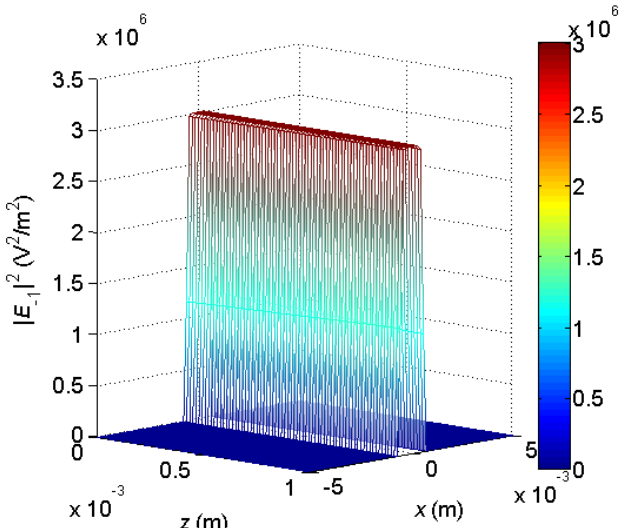

(b)

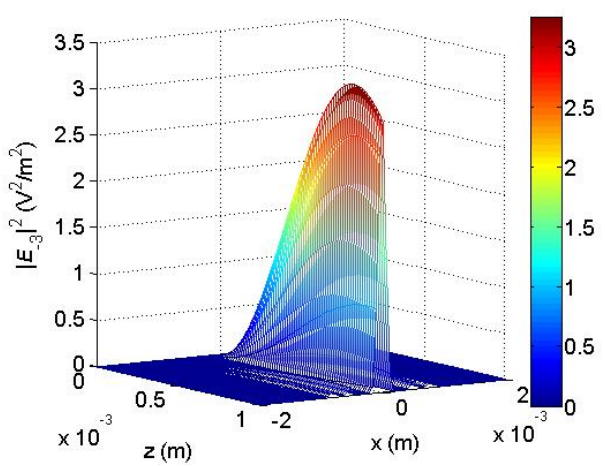

(d)

Figure 4.2: Spatial evolution of (a) first Bragg order $\left(E_{1}\right)$, (b) second Bragg order $\left(E_{-1}\right)$ (c) first non-Bragg order $\left(E_{3}\right)$, and (d) second non-Bragg order $\left(E_{-3}\right)$ in PR LN. $P_{1}=20 \mathrm{~mW}, W_{1}=3.6 \mathrm{~mm} ; P_{-1}=20 \mathrm{~mW}, W_{-1}=0.6 \mathrm{~mm} ; \theta=0.5^{\circ}$.

Theoretically from Eqs. (2.6c) - (2.6d) the widths of higher orders can be calculated. The incident Bragg orders $\left(E_{1}\right),\left(E_{-1}\right)$ have super Gaussian beam profiles. Therefore from Eq. (2.6c) the expression to calculate the width of first non-Bragg order can be written as (assuming no mode conversion):

$$
E_{3} \approx A_{1} A_{1} A_{-1}^{*} e^{-x^{16}\left(\frac{1}{W_{1}^{16}}+\frac{1}{W_{1}^{16}}+\frac{1}{W_{-1}^{16}}\right)} .
$$

When $W_{1}=6 W_{-1}$ Eq. (4.1) becomes 


$$
E_{3} \approx A_{1} A_{1} A_{-1}^{*} e^{-x^{16}\left(\frac{2}{W_{1}^{16}}+\frac{6^{16}}{W_{1}^{16}}\right)}
$$

Upon neglecting the first term in brackets in the argument of the exponent in Eq. (4.2) the waist of $E_{3}\left(W_{3}\right)$ in terms of $W_{1}$ can be written as $W_{3}=\frac{W_{1}}{6}$. As $W_{1}$ is taken as 3.6 $\mathrm{mm}$, the theoretical width of $\left|E_{3}\right|^{2}$ is $0.5746 \mathrm{~mm}$.

Similarly from Eq. (2.6d) the expression to calculate the width of second nonBragg order can be written as (assuming no mode conversion):

$$
E_{-3} \approx A_{-1} A_{-1} A_{1}^{*} e^{-x^{16}\left(\frac{1}{W_{-1}^{16}}+\frac{1}{W_{-1}^{16}}+\frac{1}{W_{1}^{16}}\right)}
$$

When $W_{1}=6 W_{-1}$ Eq. (4.3) becomes

$$
E_{-3} \approx E_{-1} E_{-1} E_{1}^{*} e^{-x^{16}\left(\frac{2\left(6^{16}\right)+1}{6^{16} W_{-1}^{16}}\right)}
$$

Upon neglecting the second term in brackets in the argument of the exponent in Eq. (4.4) the width of $E_{-3}\left(W_{-3}\right)$ in terms of $W_{-1}$ can be written as $W_{-3}=\frac{W_{-1}}{\sqrt[16]{2}}$. As $W_{-1}$ here is $0.6 \mathrm{~mm}$ the theoretical width of $\left|E_{-3}\right|^{2}$ is $0.5502 \mathrm{~mm}$. In other words if $W_{1}=$ $6 W_{-1}$ then upon approximating Eq. (4.2) and (4.4) the relation between $W_{3}$ and $W_{-3}$ can be written as

$$
W_{3}=\sqrt[16]{2} W_{-3}
$$

It is expected that the width of the intensity profile of the first non-Bragg order is greater than that of second non-Bragg order, which is in agreement with the numerically obtained values of the widths of the main lobes of these orders, which are $0.161 \mathrm{~mm}$ and $0.123 \mathrm{~mm}$, respectively. Since in this example, $W_{1}=6 W_{-1}$, the ratio of the widths of the intensity profiles of the first non-Bragg order $(+3)$ to that of the second 
non-Bragg order (-3) is approximately 1.04 , which is in reasonable agreement with the ratio of 1.30 obtained from the simulations.

Apart from this, another key observation from this analysis is that spatial side lobes are still observed in this case, even with the pump beam being much wider than the probe beam. Only by making both the incident beams wider it can be observed that the side lobes in the higher orders are suppressed, as shown below.

4.2.2 Numerical results for $P_{1}=P_{-1}=20 \mathrm{~mW} ; W_{1}=W_{-1}=2.4 \mathrm{~mm} ; \theta=2^{\circ}$. In this sub-section, the spatial variation of the higher orders has been investigated for wider flattop beam profiles, and by increasing the angle between the incident beams to $2^{\circ}$. The plots for the spatial evolution of the Bragg and non-Bragg orders, variation of on-axis intensities of the higher orders and phases are shown in Figures 4.3, 4.4 and 4.5, respectively. 


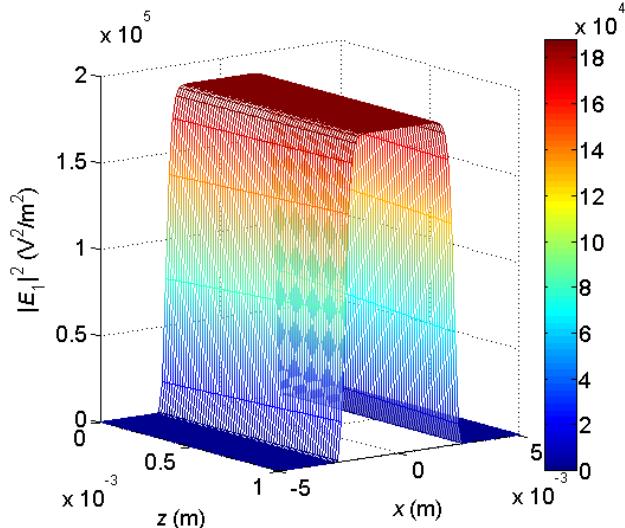

(a)

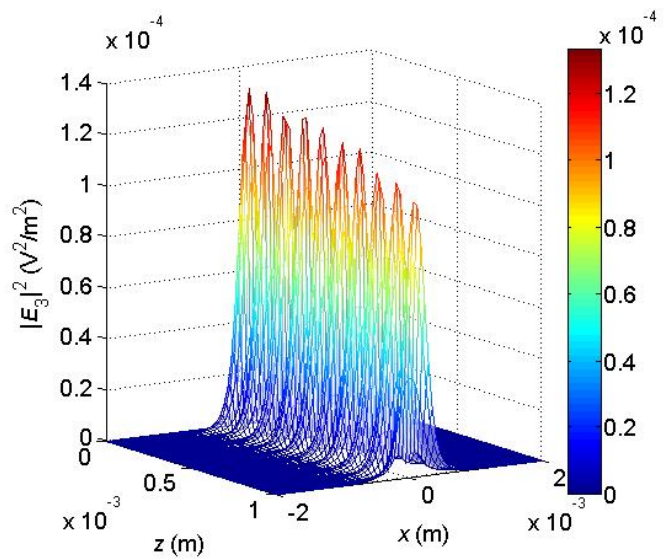

(c)

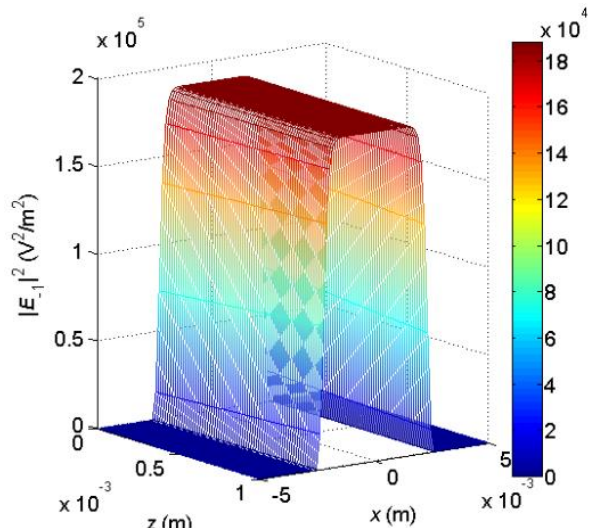

(b)

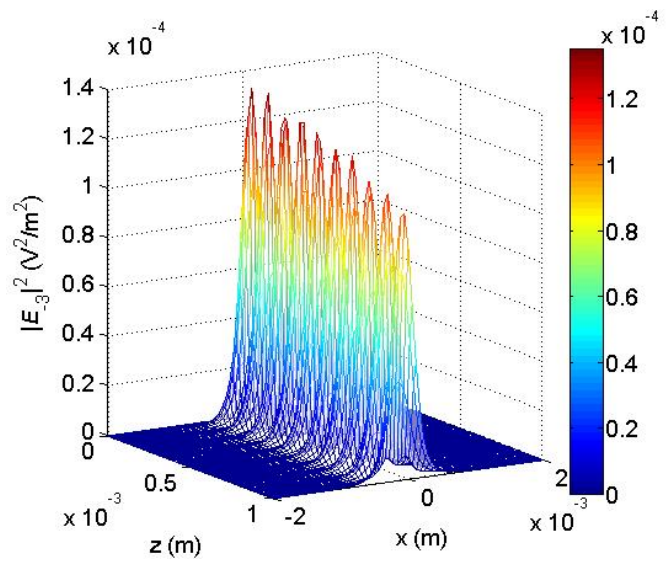

(d)

Figure 4.3: Spatial evolution of (a) first Bragg order $\left(E_{1}\right)$, (b) second Bragg order $\left(E_{-1}\right)$ (c) first non-Bragg order $\left(E_{3}\right)$, and (d) second non-Bragg order $\left(E_{-3}\right)$ in PR LN. $P_{1}=20 \mathrm{~mW}, W_{1}=2.4 \mathrm{~mm} ; P_{-1}=20 \mathrm{~mW}, W_{-1}=2.4 \mathrm{~mm} ; \theta=2^{\circ}$.

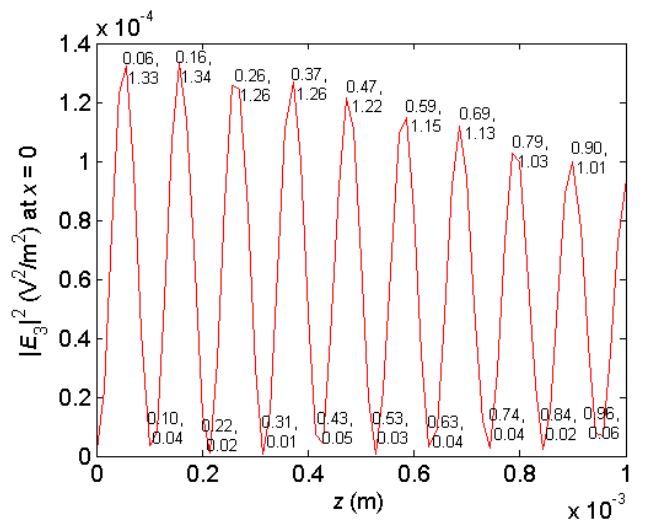

(a)

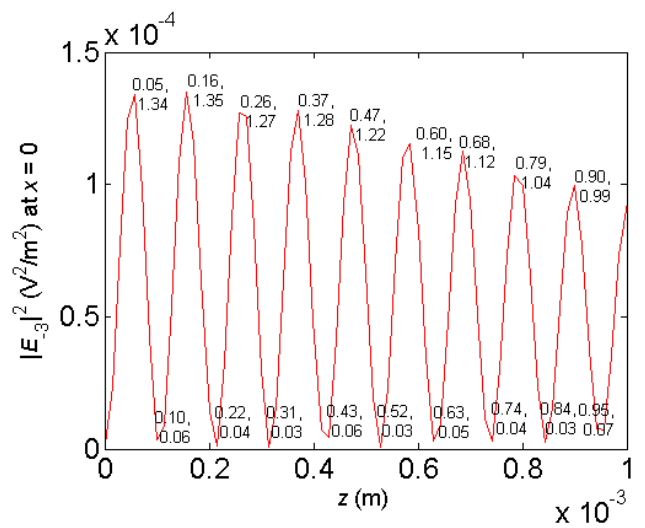

(b)

Figure 4.4: Variation of on-axis $(x=0)$ intensity of (a) first non-Bragg order $\left(E_{3}\right)$, and (b) second non-Bragg order $\left(E_{-3}\right)$ during propagation. 


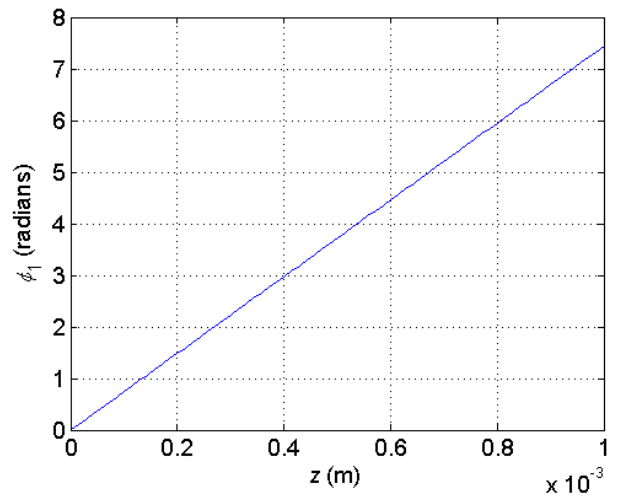

(a)

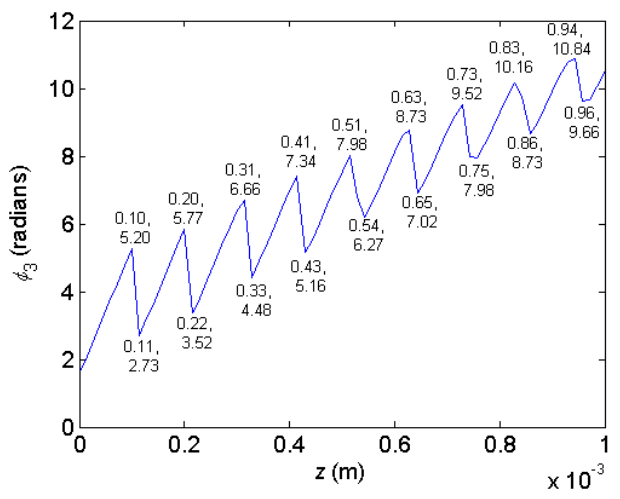

(c)

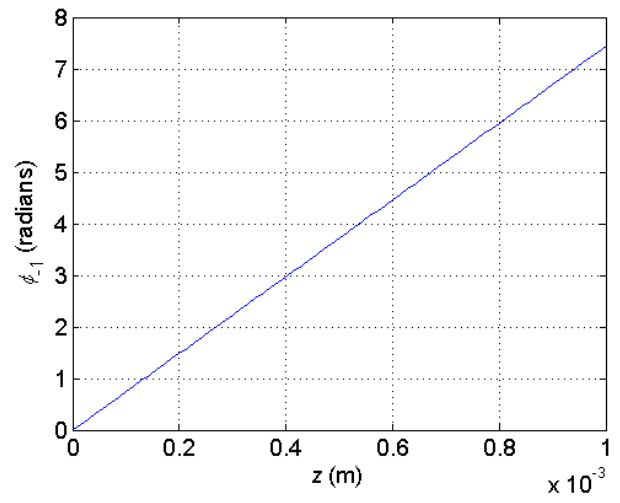

(b)

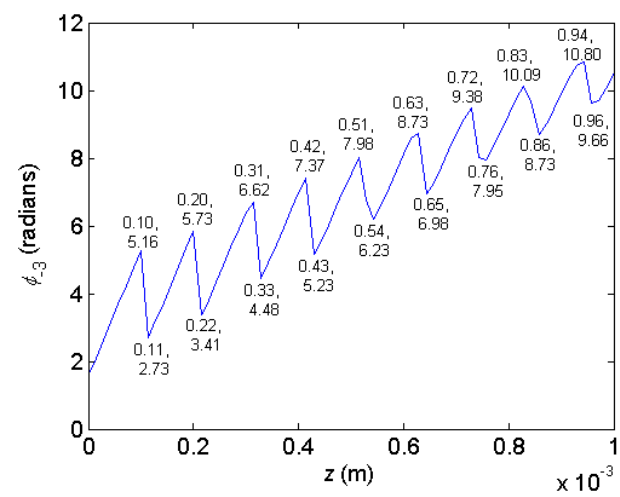

(d)

Figure 4.5: Unwrapped on-axis $(x=0)$ phase profile of (a) first Bragg order $\left(E_{1}\right)$, (b) second Bragg order $\left(E_{-1}\right)$ (c) first non-Bragg order $\left(E_{3}\right)$, and $(d)$ second non-Bragg $\operatorname{order}\left(E_{-3}\right)$ during propagation.

Once again, from Figure 4.3 it is evident that higher or non-Bragg orders are generated at the expense of energy from +1 and -1 (Bragg) orders. Also, the intensities of the first and second non-Bragg orders are identical, because the incident beams have identical powers. Interestingly from Figure 4.3 it can be observed that there are no side lobes in higher orders. In other words the effect of mode conversion is suppressed by using larger widths for both incident profiles. Unlike Gaussian beam incidence, if the incident Bragg orders have wider flattop profiles then the side lobes in the higher orders can be suppressed. This may be possibly due to the fact that as the incident beam profiles here are flattops, the higher order beams after their generation may encounter less asymmetric induced refractive index profile than with Gaussian beam incidence. 
Apart from this, there is a quasi-periodic (and identical) axial variation of the higher order intensities as they propagate through the crystal which is similar to that observed for Gaussian beam incidence. The period of the axial variation can be determined from Figure 4.4 which is approximately $0.1 \mathrm{~mm}$. Furthermore, by examining Figures 4.3 and 4.2 it can be surmised that this period increases with decreasing interaction angle. Also from Figure 4.5 it is evident that the phases of the non-Bragg orders start from $\pi / 2$ and they also show a quasi-periodic variation with the same period as that of the intensity. On the other hand, the phases of the Bragg orders linearly increase from zero.

\subsection{Numerical Analysis by Varying the Incident Beam Profiles}

In this Section, numerical analysis is performed by varying the incident beam parameters along with incident beam profiles. A combination of Gaussian and super Gaussian profiles are used as pump (+1) and probe $(-1)$ beams, respectively. Numerical simulations are performed for two different cases:

$$
\begin{aligned}
& \text { 1) } P_{1}=P_{-1}=20 \mathrm{~mW} ; W_{1}=3.6 \mathrm{~mm}, W_{-1}=0.6 \mathrm{~mm} ; \theta=0.5^{\circ} \text {. } \\
& \text { 2) } P_{1}=P_{-1}=20 \mathrm{~mW} ; W_{1}=W_{-1}=2.4 \mathrm{~mm} ; \theta=2^{\circ} .
\end{aligned}
$$

\footnotetext{
4.3.1 Numerical results for $P_{1}=P_{-1}=20 \mathrm{~mW} ; W_{1}=3.6 \mathrm{~mm}, W_{-1}=$ $0.6 \mathrm{~mm} ; \theta=0.5^{\circ}$.

In this sub-section a wider Gaussian and a narrow super Gaussian profiles are used as pump and probe beams respectively. The incident beam profiles and the intensity variation plots are shown in Figures 4.6 and 4.7 respectively.
} 


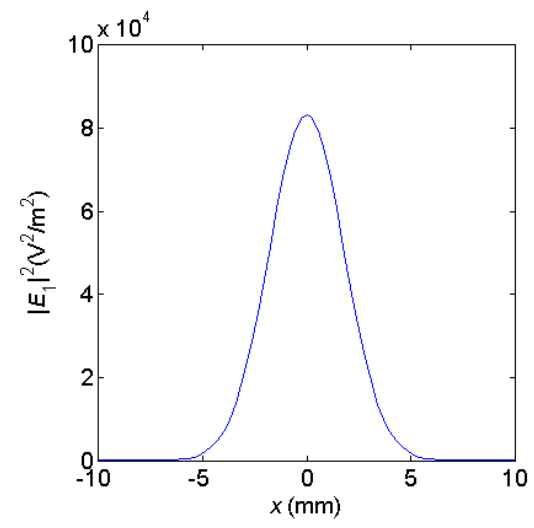

(a)

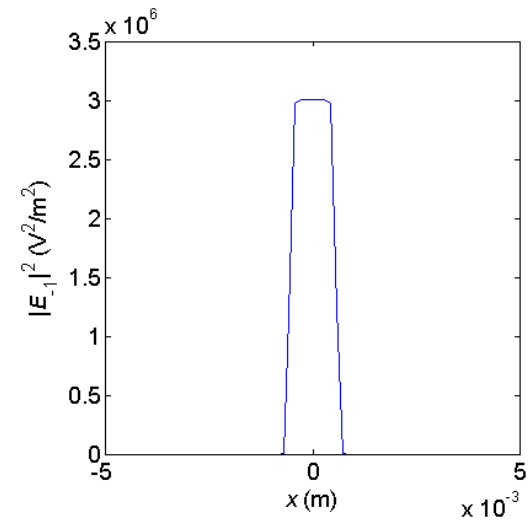

(b)

Figure 4.6: Gaussian and flattop beam profiles of incident (a) pump (reference) and (b) probe (object) beams. $P_{1}=20 \mathrm{~mW}, W_{1}=3.6 \mathrm{~mm} ; P_{-1}=20 \mathrm{~mW}, W_{-1}=0.6 \mathrm{~mm}$.

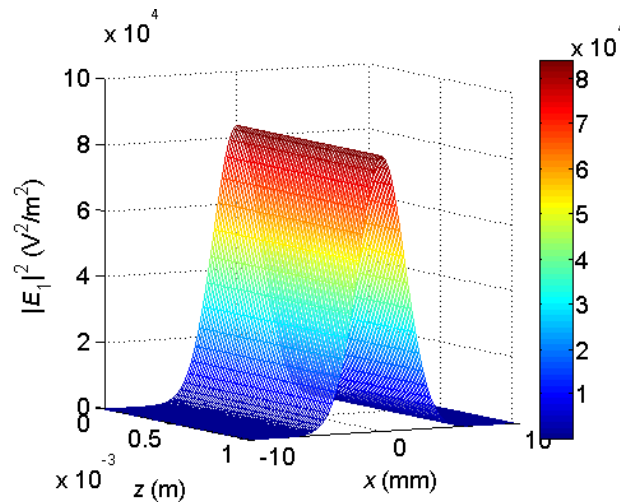

(a)

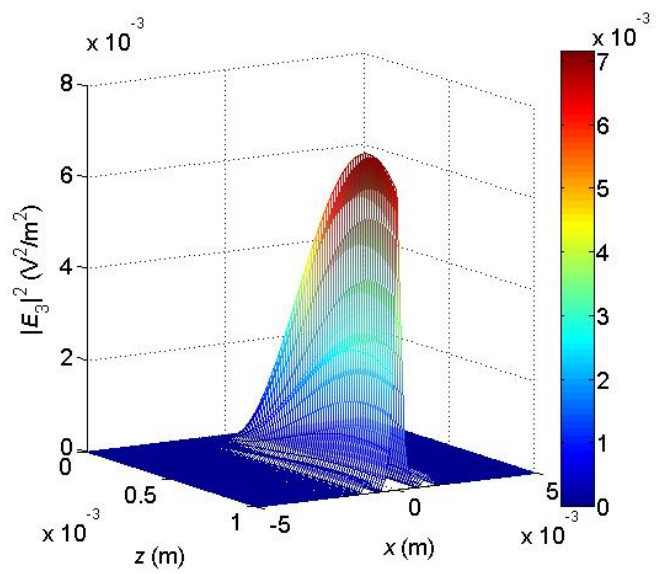

(c)

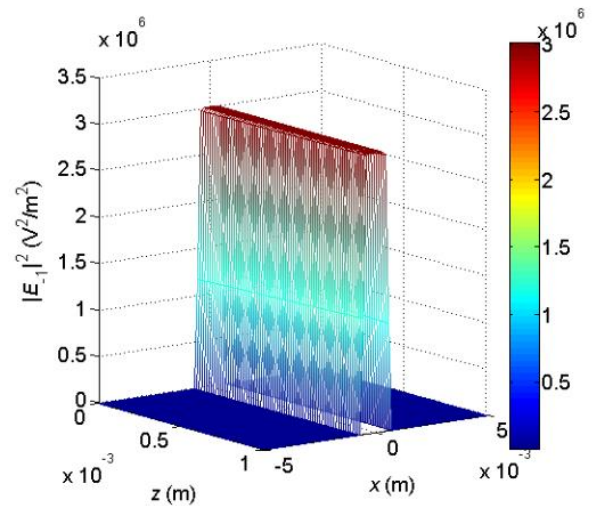

(b)

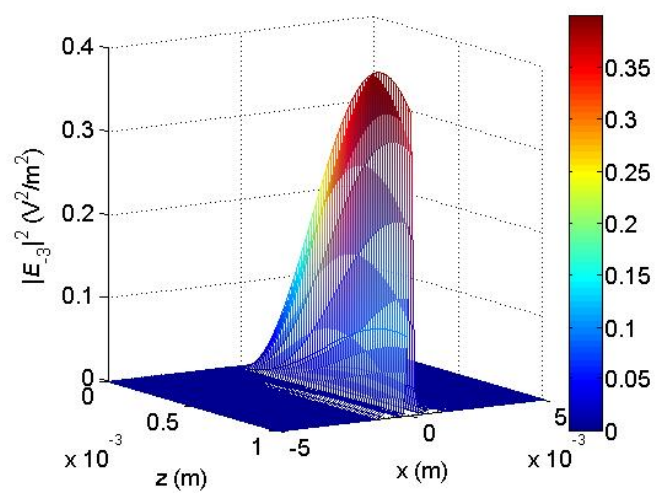

(d)

Figure 4.7: Spatial evolution of (a) first Bragg order $\left(E_{1}\right)$, (b) second Bragg order $\left(E_{-1}\right)$ (c) first non-Bragg order $\left(E_{3}\right)$, and (d) second non-Bragg order $\left(E_{-3}\right)$ in PR LN. $P_{1}=20 \mathrm{~mW}, W_{1}=3.6 \mathrm{~mm} ; P_{-1}=20 \mathrm{~mW}, W_{-1}=0.6 \mathrm{~mm} ; \theta=0.5^{\circ}$. 
Theoretical estimation of the widths of the higher orders can be attempted from Eqs. (2.6c) - (2.6d). However, since $E_{1}$ has Gaussian profile and $E_{-1}$ has a flattop or super-Gaussian beam profile, the expressions to calculate the width of first and second non-Bragg orders can be written as (assuming no mode conversion):

$$
E_{3} \approx A_{1} A_{1} A_{-1}^{*} e^{-x^{2}\left(\frac{1}{w_{1}^{2}}+\frac{1}{W_{1}^{2}}+\frac{x^{8}}{W_{-1}^{16}}\right)}
$$

and

$$
E_{-3} \approx E_{-1} E_{-1} E_{1}^{*} e^{-x^{2}\left(\frac{x^{8}}{W_{-1}^{16}}+\frac{x^{8}}{W_{-1}^{16}}+\frac{1}{W_{1}^{2}}\right)} .
$$

Theoretically from Eqs. (4.6) and (4.7) the widths of the higher orders cannot be estimated. But as the width of first Bragg order is 6 times greater than the width of second Bragg order, it is expected that the width of the intensity profile of the first nonBragg order is also greater than second non-Bragg order, which is in agreement with the numerically obtained values of the widths of the main lobes of these orders, which are $0.235 \mathrm{~mm}$ and $0.145 \mathrm{~mm}$, respectively. Also as before, even though the incident beam profiles are different there is mode conversion in the higher non-Bragg orders.

4.3.2 Numerical results for $P_{1}=P_{-1}=20 \mathrm{~mW} ; W_{1}=W_{-1}=2.4 \mathrm{~mm} ; \theta=2^{\circ}$. In this sub-section numerical analysis is performed for two different wider beam profiles by increasing the angle between them to $2^{\circ}$. The variations of the intensity profiles, on-axis intensities and phases are shown in Figures 4.8, 4.9, and 4.10 respectively. 


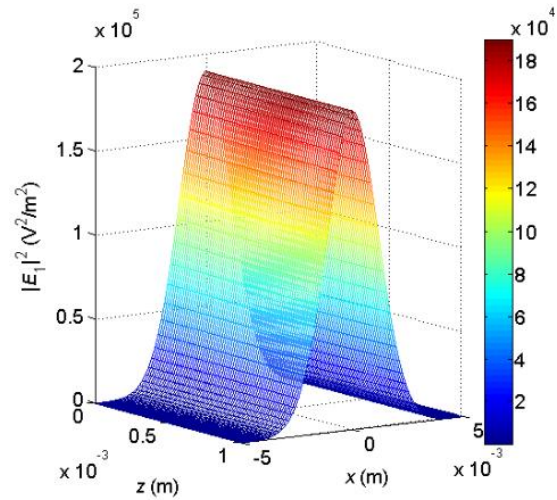

(a)

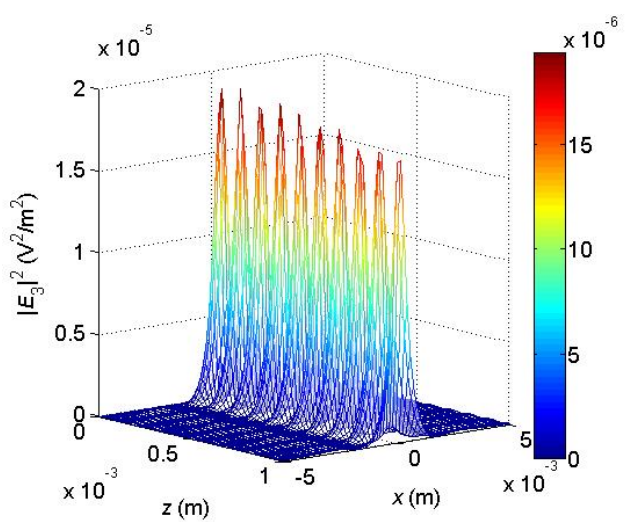

(c)

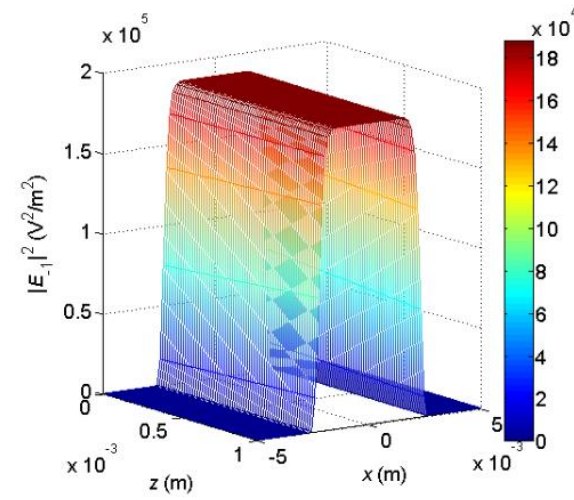

(b)

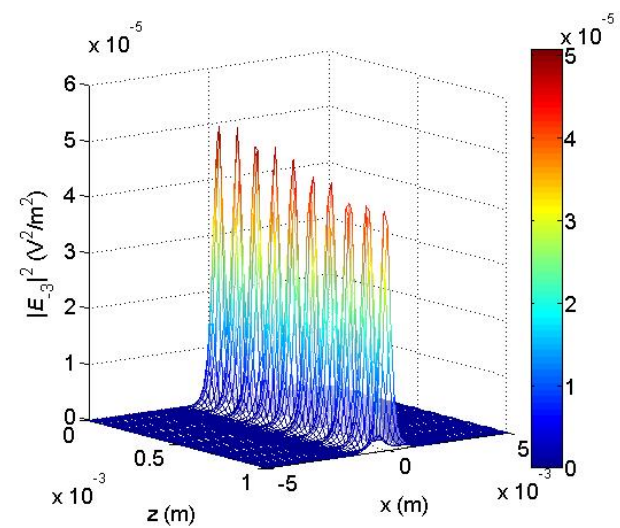

(d)

Figure 4.8: Spatial evolution of (a) first Bragg order $\left(E_{1}\right)$, (b) second Bragg order $\left(E_{-1}\right)$ (c) first non-Bragg order $\left(E_{3}\right)$, and (d) second non-Bragg order $\left(E_{-3}\right)$ in PR LN. $P_{1}=20 \mathrm{~mW}, W_{1}=2.4 \mathrm{~mm} ; P_{-1}=20 \mathrm{~mW}, W_{-1}=2.4 \mathrm{~mm} ; \theta=2^{\circ}$.

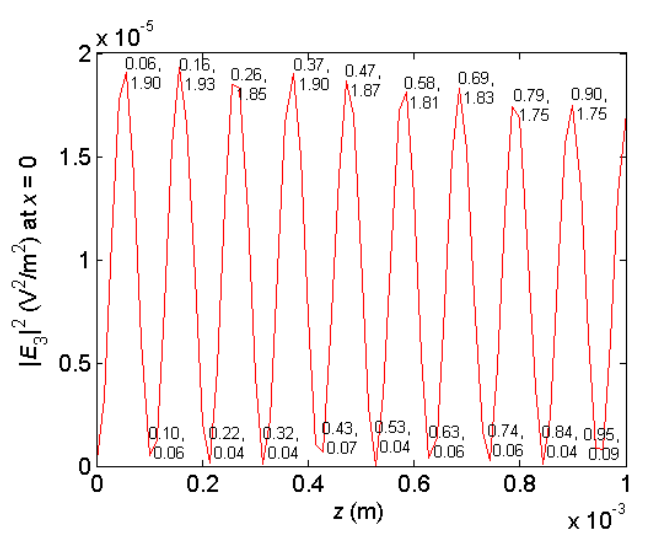

(a)

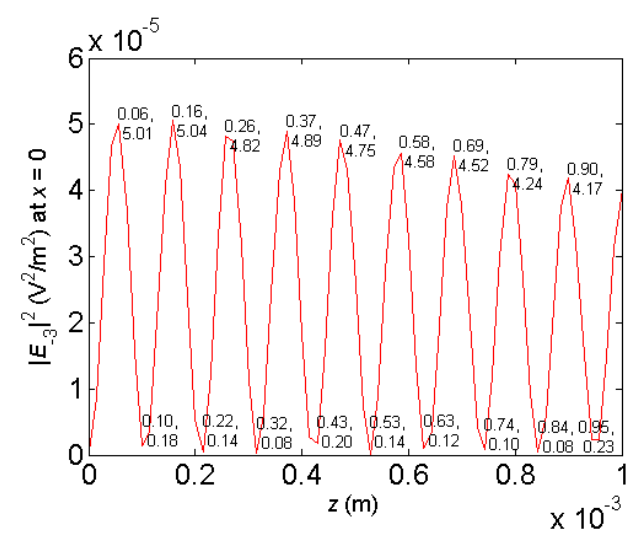

(b)

Figure 4.9: Variation of on-axis $(x=0)$ intensity of (a) first non-Bragg order $\left(E_{3}\right)$, and (b) second non-Bragg order $\left(E_{-3}\right)$ during propagation. 


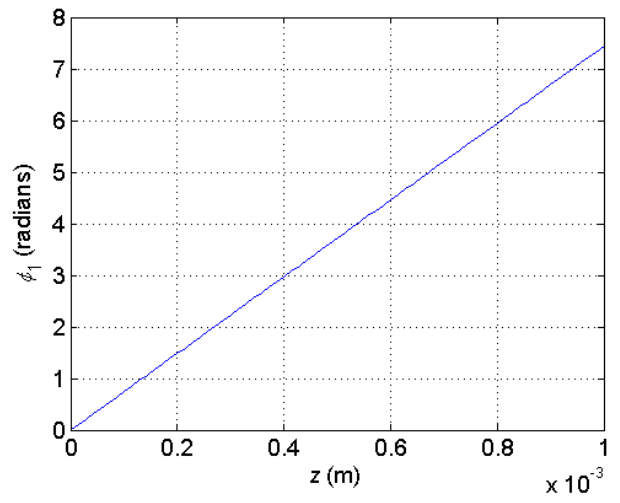

(a)

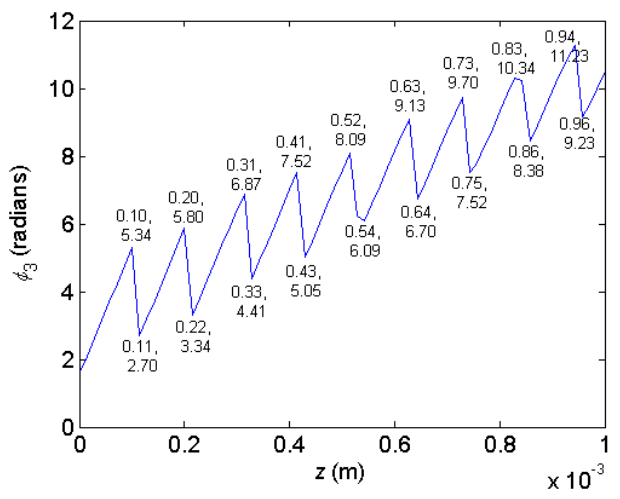

(c)

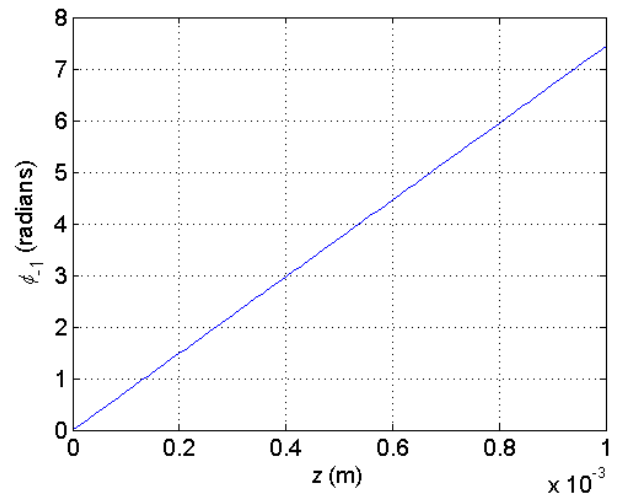

(b)

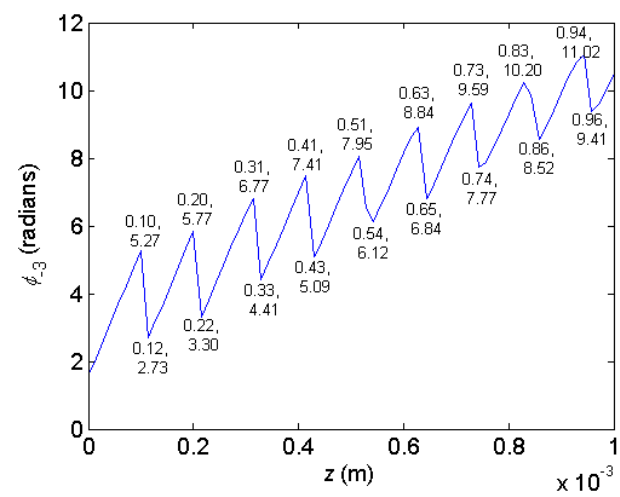

(d)

Figure 4.10: Unwrapped on-axis ( $x=0$ ) phase profile of (a) first Bragg order $\left(E_{1}\right)$, (b) second Bragg order $\left(E_{-1}\right)(\mathrm{c})$ first non-Bragg order $\left(E_{3}\right)$, and (d) second non-Bragg order $\left(E_{-3}\right)$ during propagation.

Though the powers of the two incident beams are equal, the peak intensities of the first and second non-Bragg orders are different, because of the fact that the profiles of the incident beams are different. Interestingly from Figure 4.8 it can be observed that there are no side lobes in the higher orders. Therefore it can be concluded that though the profiles of the incident beams are different, if the incident Bragg orders are wider, then the effect of the mode conversion of incident beam profiles during the evolution of higher orders can be suppressed.

Apart from this, though the peak intensities of the non-Bragg orders are different the simulations confirm our earlier postulates that the spatial period of the variation of 
the higher orders on-axis intensities and their phases decreases with increasing interaction angle. Also the variation of the phases of the various orders are similar to that in the previous example (see Section 4.2.2) and seem to be relatively unaffected with change in the shape (profile) of the incident beams.

\subsection{Conclusion}

In this Chapter the effect of mode conversion in higher orders has been investigated by using a combination of Gaussian and super Gaussian profiles along with super-Gaussians (flattops) alone. From this analysis it can be concluded that irrespective of the incident beam profiles i.e. flattops alone or a combination of Gaussian and a flattop, an increased width suppresses the generation of spatial sidelobes. Interestingly even when the incident beam profiles are flattops, the profiles of the higher orders do not remain super Gaussian. The reason for this is under investigation. It is surmised that the complex interactions giving rise to the spatial frequencies of higher orders may be responsible for spatial filtering of these orders. The non-Bragg orders are similar to forward phase conjugation and forward phase amplification [11]. Also it has been found that regardless of the incident beam profiles i.e. Gaussians or flattops or a combination of Gaussian and flattop, when the external angle between the incident beams is increased, the "launch" angle for the non-Bragg orders also increased, resulting in a smaller value of the repetition period. 


\section{CHAPTER V}

\section{CONCLUSION AND FUTURE WORK}

\subsection{Conclusion}

In this thesis, an exact study is performed to study the spatial evolution of Bragg and non-Bragg orders in PR LN for different types of beam profiles such as Gaussians, flattops and a combination of both. Expressions for the complex optical field inside a PR material for Bragg and non-Bragg orders are derived by decomposing them into their respective angular plane wave spectra. The obtained equations are then numerically solved in MATLAB ${ }^{\odot}$ using Range-Kutta methods for a variety of incident beam profiles. A thorough numerical analysis is performed by varying all the incident beam parameters such as incident beam powers, beam waists and also the angle between incident beams.

For Gaussian beam incidence, higher or non-Bragg orders shows evidence of spatial sidelobes indicating mode conversion of the incident beam profiles, possibly due to the higher orders seeing an asymmetric induced refractive index profile and therefore not being optimally guided through the medium. The results are similar when using super-Gaussians or flat-top profiles as the incident beams, as well as when one of the incident beams is Gaussian and the other a super-Gaussian. Spatial sidelobes can be reduced by increasing the widths of the incident beams. When the interaction angle 
between the incident beams is increased, the period of spatial evolution of the higher orders decrease, possibly due to the increased launch angle of the beams. This is accompanied by a decrease in the peak intensities, possibly due to decrease in the interaction length.

\subsection{Future Work}

In this Section, continuation of some parts of this thesis is described as future work. From the results of numerical analysis it can be concluded that for Gaussian beam incidence, higher or non-Bragg orders shows evidence of mode conversion of incident beam profiles. While preliminary experiments have been performed, future experiments are planned to exactly monitor the shapes of the non-Bragg orders, both at the exit plane of the PR material using imaging, as well as in the far-field. Also the physical reason behind this phenomenon will be investigated with the help of PR physics.

Apart from this, it has been found that higher orders vary periodically along $z$ axis with variation in the angle between incident beams. The physical reason behind this phenomenon will also be investigated as part of future work.

The numerical modeling in this work has been performed assuming only the photovoltaic effect for the PR material. Extension to the case where the diffusion effect is dominant and the general case of diffusion, drift and photovoltaic effect will be performed in the future.

Finally, the numerical technique will be extended to arbitrary object beam profiles, with applications to dynamic PSDH and DHI in mind. 


\section{BIBLIOGRAPHY}

[1] U. Abeywickrema, P. Banerjee, A. Kota, A. Lakhtakia, and S. Swiontek, “3d fingerprint analysis using transmission-mode multi-wavelength digital holographic topography," Proc. SPIE 9771, 1-7 (2016).

[2] P. Banerjee, G. Nehmetallah, U. Abeywickrema, S. Lyuksyutov, and N. Kukhtarev, "Non-Bragg diffraction orders in holographic recording and its application to one-shot phase-shifting holographic interferometry," Proc. SPIE 8644, 864402-1-864402-9 (2013).

[3] N. Kukhtarev, T. Kukhtareva, P. Banerjee, and G. Nehmetallah, "Holographic imaging and interferometry with non-Bragg diffraction orders in volume gratings," DTu3C.3, Topical meeting in Digital Holography and Three Dimensional Imaging (2012).

[4] P. Banerjee, G. Nehmetallah, N. Kukhtarev, and S. Praharaj, "Determination of model airplane attitudes using dynamic holographic interferometry," Appl. Opt. 47, 3877-3885 (2008).

[5] T. Matsumoto, T. Watanabe, A. Kojima, N. Kato, M. Tamiwa, and M. Baba, "Deformation analysis of the human femur by holographic interferometry," 29th Annual International Conference of the IEEE Engineering in Medicine and Biology Society, 4699-4702 (2007).

[6] M. Gesualdi, M. Mori, M. Muramatsu, E. Liberti, and E. Munin, "Phase-shifting real-time holographic interferometry applied to load transmission evaluation in dried human skull,” Appl. Opt. 46, 5419-5429 (2007). 
[7] P. Yeh, Introduction to Photorefractive Nonlinear Optics. (Wiley, New York, 1993).

[8] L. Au and L. Solymar, "Higher diffraction orders in photorefractive materials," IEEE J. Quantum Elect. 24, 162-168 (1988).

[9] J. Liu, P. Banerjee, and Q. Wang Song, "Role of diffusive, photovoltaic, and thermal effects in beam fanning in $\mathrm{LiNbO}_{3}$," J. Opt. Soc. Am. B 11, 1688-1693 (1994).

[10] U. Abeywickrema and P. Banerjee, "Phase-shifting holography using Bragg and non-Bragg orders in photorefractive lithium niobate," Proc. SPIE 9200, $1-8(2014)$.

[11] P. Banerjee, U. Abeywickrema, G. Nehmetallah, S. Lyuksyutov, and N. Kukhtarev, "Applications of Bragg and non-Bragg orders in holography and interferometry," Proc. SPIE 8883, 1-9 (2013).

[12] T. Credelle and F. Spong, "Thermoplastic media for holographic recording," Proc. SPIE 130, 619-633 (1996).

[13] G. Nehmetallah and P. Banerjee, "Applications of digital and analog holography in three dimensional imaging," Adv. Opt. Photon. 4, 472-553 (2012).

[14] U. Abeywickrema, "Applications of Induced Gratings in Nonlinear Media," Ph.D. Dissertation, University of Dayton, 2015.

[15] L. Solymar, D. Webb, and A. Grunnet-Jepsen, The Physics and Applications of Photorefractive Materials. (Oxford, New York 1996). 
[16] I. Kaminow, An Introduction to Electro-optic Devices. (Academic, New York 1997). 


\section{APPENDIX}

\section{Selected MATLAB Codes Used in This Work}

\section{Generation of the Angular Spectrum of Incident Beam Profiles (Chapter 3, 4)}

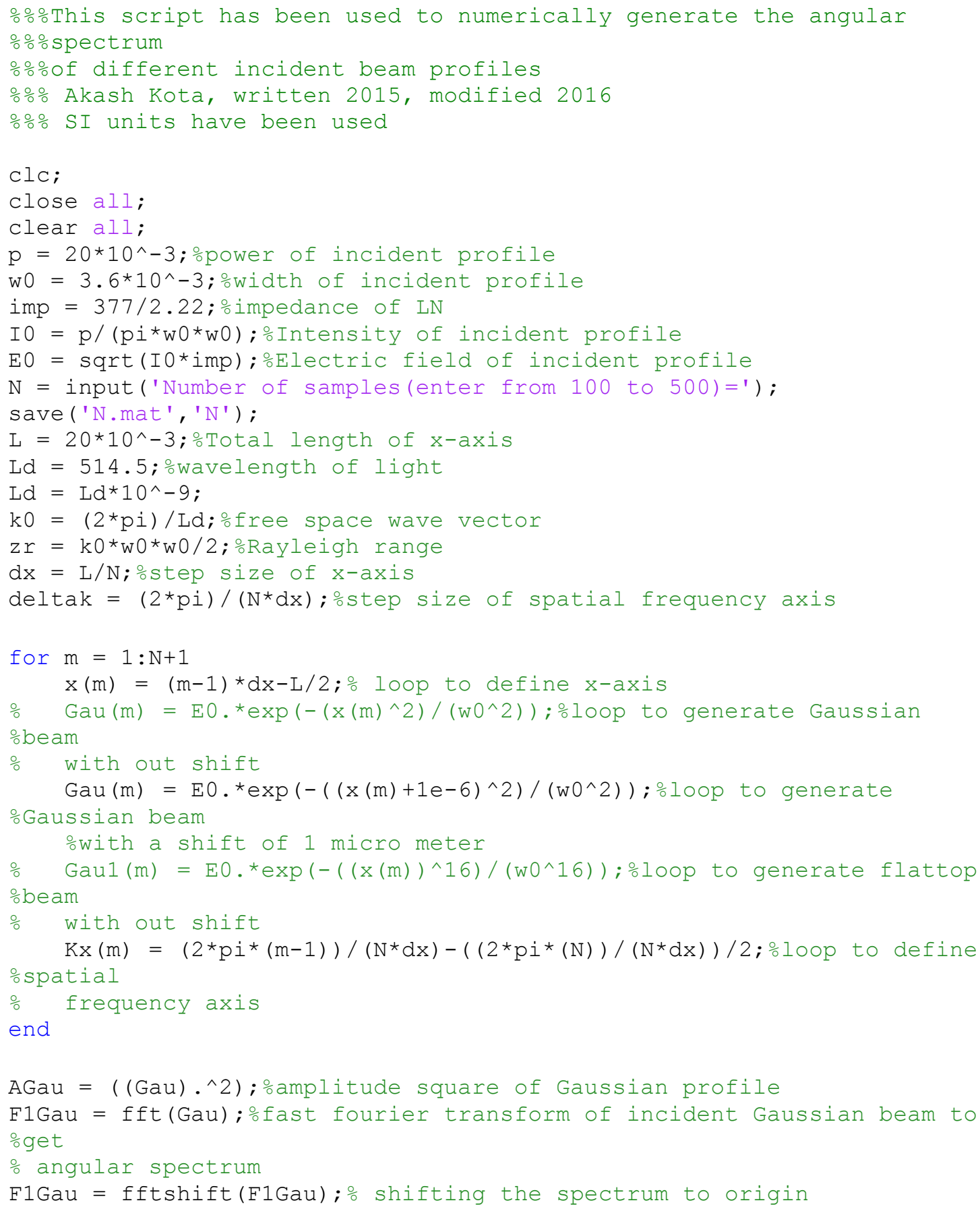




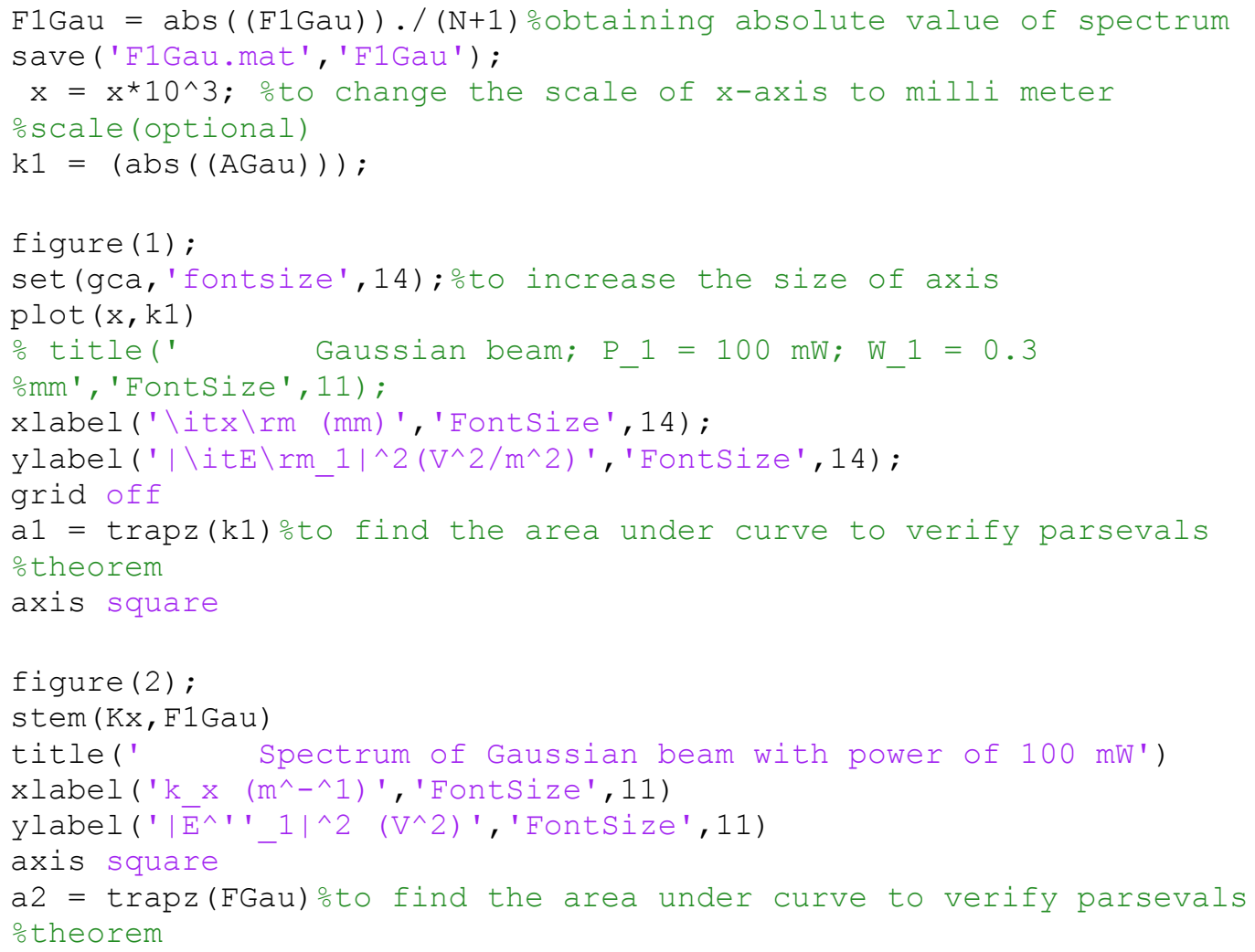

Solving Coupled Differential Equations for Bragg and Non-Bragg Orders Using

\section{Range-Kutta Methods}

응 This script solves the coupled differential equations 응ㅌs. (2.9a-d) are numerically solved for different incident 으아일. The number of equations need to be solved depends on \%응 number of angular components. In this work a total of 응구 angular components are taken resulting in 284 coupled equations .

응ue to space constraint all the equations are not given in this thesis.

응ㅁt a sample code with 3 angular components is given.

$\mathrm{ClC}$;

close all;

clear all;

global a $\mathrm{K}$ k0 z w Cl

c1 $=-2.1 e-11 ; \%$ Value of photovoltaic coefficient

$\mathrm{a}=2 *(2 * \mathrm{pi} / 360)$; $\%$ external angle between incident beams

$\mathrm{w}=514.5 e-9 ; \%$ wavelength of light used

$\mathrm{k} 0=2 * \mathrm{pi} / \mathrm{w}$; $\%$ free space wave vector

$\mathrm{K}=2 * \mathrm{k} 0 * \sin (\mathrm{a})$; ㅇaㅁitude of Grating vector

load ('N.mat', 'N');

$\mathrm{z}=(0: 1 / \mathrm{N}: 1) * 10^{\wedge}-3 ; \%$ Thickness of crystal

load('FlGau.mat', 'FlGau'); $\%$ loading components of first Bragg order load('F2Gau.mat', 'F2Gau'); oloading components of second Bragg order $\mathrm{E} 10=\operatorname{F1Gau}((\mathrm{N} / 2)+1)$

$\mathrm{E} 11=\operatorname{F1Gau}((\mathrm{N} / 2)+2) ; \mathrm{E} 111=\operatorname{F1Gau}(\mathrm{N} / 2) ;$

$\mathrm{E} 20=\operatorname{F} 2 \operatorname{Gau}((\mathrm{N} / 2)+1)$

$\mathrm{E} 21=\mathrm{F} 2 \mathrm{Gau}((\mathrm{N} / 2)+2) ; \mathrm{E} 2 \_11=\mathrm{F} 2 \mathrm{Gau}(\mathrm{N} / 2) ;$

$\mathrm{E} 30=0 ; \mathrm{E} 31=0 ; \mathrm{E} 3 \_11=0$; 


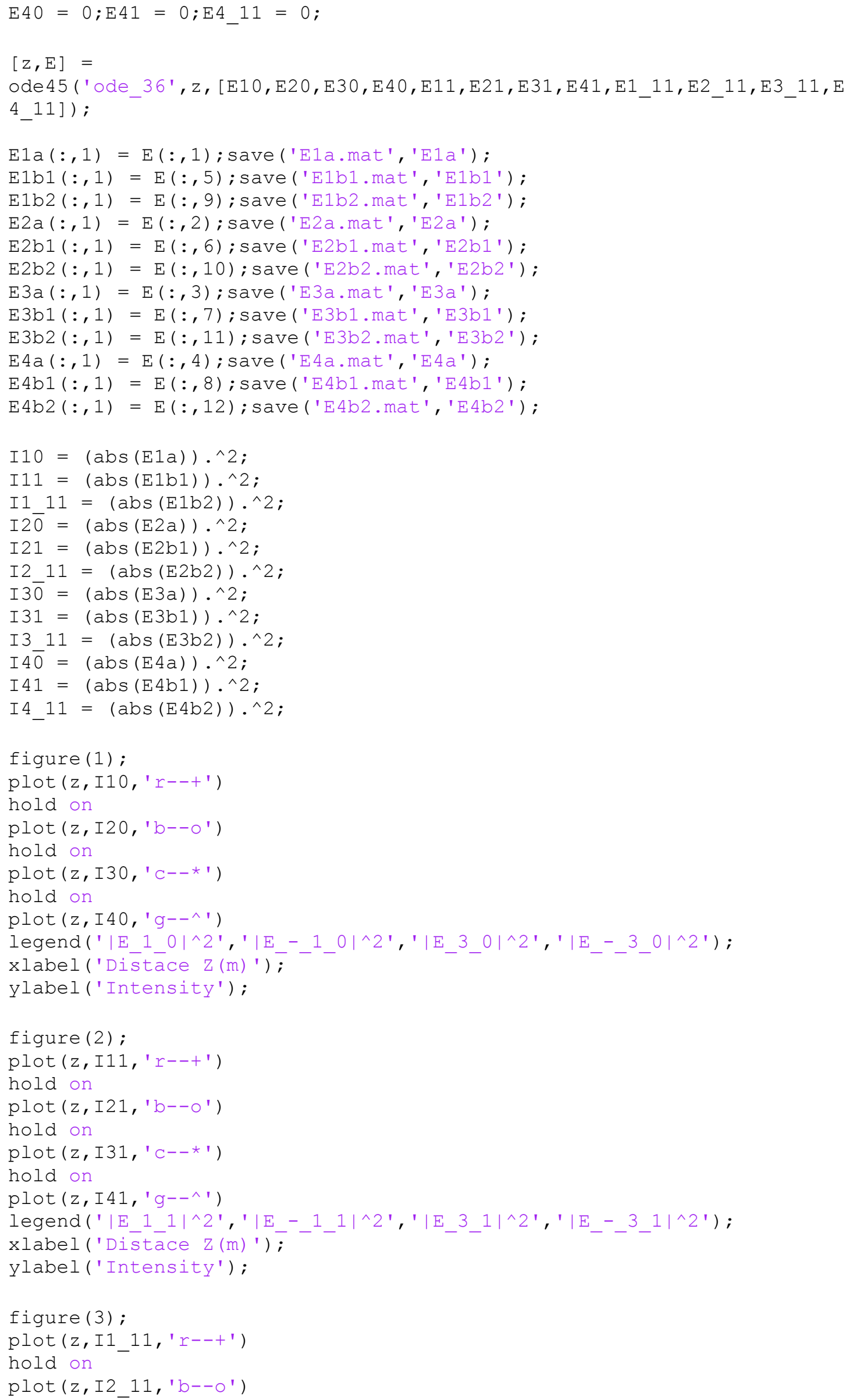


hold on

plot (z,I3_11, 'C--*')

hold on

plot $\left(\mathrm{z}, \mathrm{I} 4 \mathrm{11}, \mathrm{g}^{--\wedge}\right.$ ')

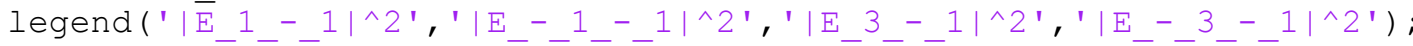

xlabel ('Distāēe Z (m)') ;

ylabel ('Intensity');

\section{Function for Solving the Coupled Differential Equations using ODE45}

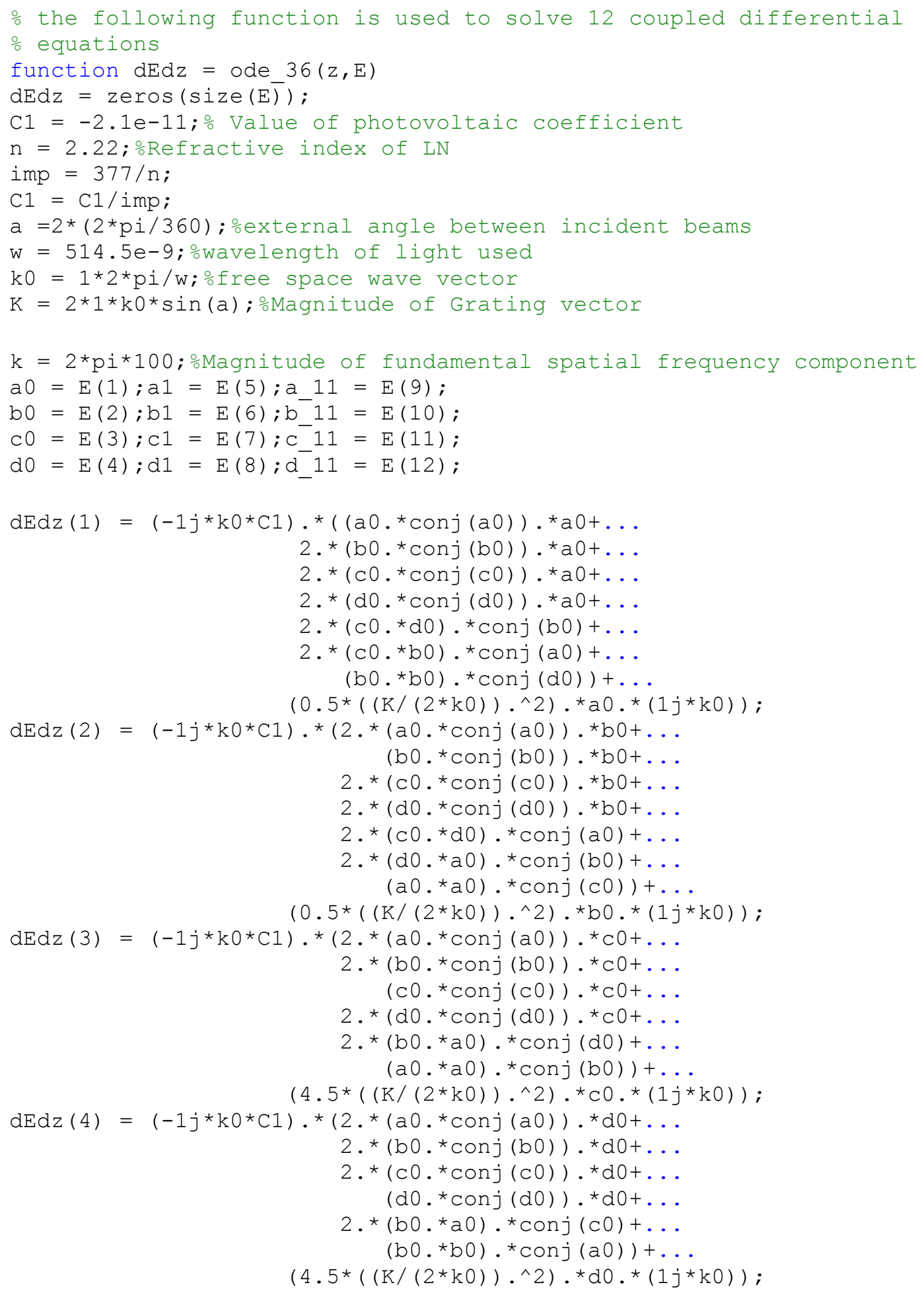




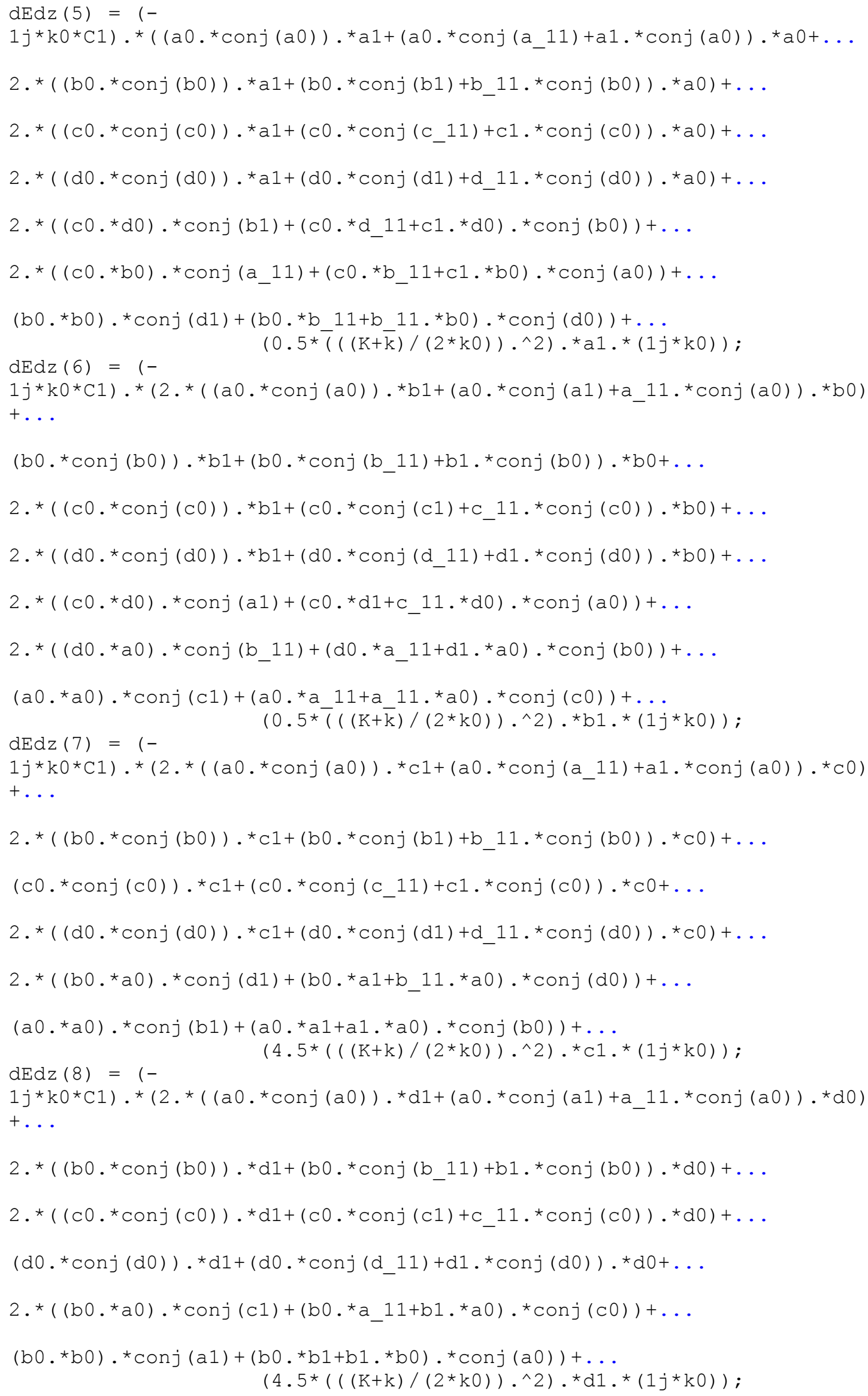




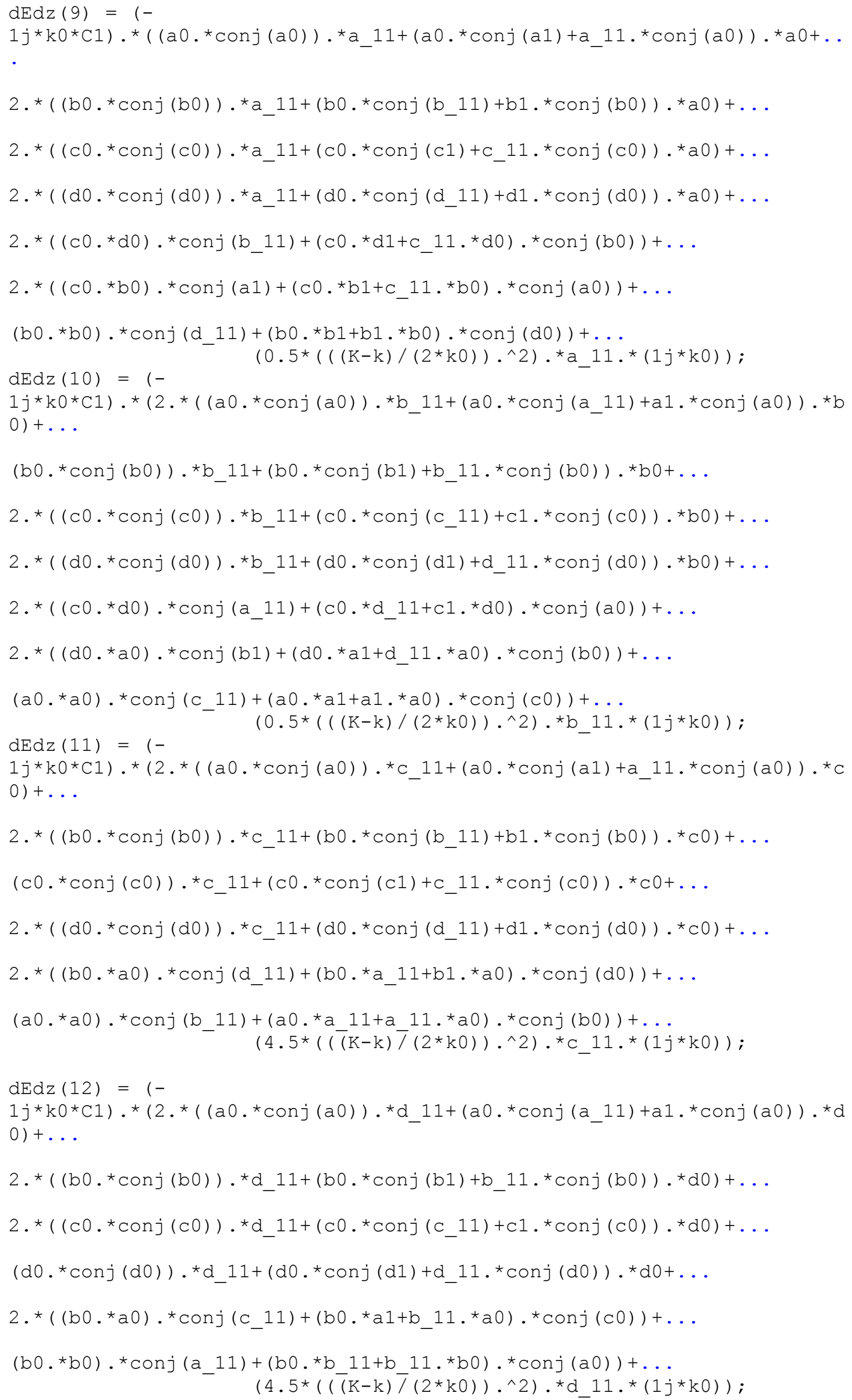

